\title{
DEVELOPMENT OF MEASUREMENT METHODS FOR APPLICATION TO A WIND TUNNEL TEST OF AN ADVANCED TRANSPORT MODEL
}

\author{
A Thesis \\ presented to \\ the Faculty of California Polytechnic State University, \\ San Luis Obispo
}

\author{
In Partial Fulfillment \\ of the Requirements for the Degree \\ Master of Science in Aerospace Engineering \\ by \\ Robert Schaefer Ehrmann
}

August 2010 
(C) 2010

Robert Schaefer Ehrmann

ALL RIGHTS RESERVED 


\section{COMMITTEE MEMBERSHIP}

TITLE:

AUTHOR:

DATE SUBMITTED:

COMMITTEE CHAIR:

COMMITTEE MEMBER:

COMMITTEE MEMBER:

COMMITTEE MEMBER:
Development of Measurement Methods for Application to a Wind Tunnel Test of an Advanced Transport Model

Robert Schaefer Ehrmann

August 2010

Dr. Kristina Jameson, Assistant Professor

Dr. David Marshall, Associate Professor

Dr. Rob McDonald, Assistant Professor

Dr. Russell Westphal, Professor 


\title{
ABSTRACT \\ DEVELOPMENT OF MEASUREMENT METHODS FOR APPLICATION TO A WIND TUNNEL TEST OF AN ADVANCED TRANSPORT MODEL
}

\author{
Robert Schaefer Ehrmann
}

California Polytechnic State University, San Luis Obispo is currently working towards developing a Computational Fluid Dynamics (CFD) database for future code validation efforts. Cal Poly will complete a wind tunnel test on the Advanced Model for Extreme Lift and Improved Aeroacoustics (AMELIA) in the National Full-Scale Aerodynamics Complex (NFAC) 40 foot by 80 foot wind tunnel at NASA Ames Research Center in the summer of 2011. The development of two measurement techniques is discussed in this work, both with the objective of making measurements on AMELIA for CFD validation.

First, the work on the application of the Fringe-Imaging Skin Friction (FISF) technique to AMELIA is discussed. The FISF technique measures the skin friction magnitude and direction by applying oil droplets on a surface, exposing them to flow, measuring their thickness, and correlating their thickness to the local skin friction. The technique has the unique ability to obtain global skin friction measurements. A two foot, nickel plated, blended wing section test article has been manufactured specifically for FISF. The model is illuminated with mercury vapor lamps and imaged with a Canon 50D with a $546 \mathrm{~nm}$ bandpass filter. Various tests are applied to the wing in order to further characterize uncertainties related with the FISF technique. Human repeatability has uncertainties of $\pm 2.3 \%$ of fringe spacing and $\pm 2.0^{\circ}$ in skin friction vector direction, while image post processing yields $\pm 25 \%$ variation in skin friction coefficient. A method for measuring photogrammetry uncertainty is developed. The effect of filter variation and test repeatability was found to be negligible. A validation against a Preston tube was found to have $1.8 \%$ accuracy.

Second, the validation of a micro flow measurement device is investigated. Anemometers have always had limited capability in making near wall measurements, driving the design of new devices capable of measurements with increased wall proximity. Utilizing a thermocouple boundary layer rake, wall measurements within 0.0025 inches of the surface have been made. A Cross Correlation Rake (CCR) has the advantage of not requiring calibration but obtaining the same proximity and resolution as the thermocouple boundary layer rake. The flow device utilizes time of flight measurements computed via cross correlation to calculate wall velocity profiles. The CCR was designed to be applied to AMELIA to measure flow velocities above a flap in a transonic flow regime. The validation of the CCR was unsuccessful. Due to the fragile construction of the CCR, only one data point at 0.10589 inches from the surface was available for validation. The subsonic wind tunnel's variable frequency drive generated noise which could not be filtered or shielded, requiring the use of a flow bench for validation testing. Since velocity measurements could not be made in the flow bench, a 
comparison of a fast and slow velocity was made. The CCR was not able to detect the difference between the two flow velocities. Currently, the CCR cannot be applied on AMELIA due to the unsuccessfully validation of the device. 


\section{ACKNOWLEDGMENTS}

I would like to acknowledge the NASA Research Announcement award under Contract \#NNL07AA55C, with technical monitors Craig Hange and Clif Horne, who supported this research.

Much of my work has been made possible with generous time from various NASA employees. First and foremost, a large thanks to Gregory Zilliac for constant support with the FISF software. You graciously taught me the technique on FAITH and allowed me to observe the CMR. Your help and assistance are greatly appreciated. Thank you also to David Driver for continued correspondence and aid in preparation for the AMELIA test. A thanks to the NASA Ames Fluid Mechanics Laboratory for assisting in my early research as well. Gustave Fralick and John Wrbanek of NASA Glenn proved invaluable resources for the application of the CCR.

An extremely special thanks to Dr. Westphal. You always allowed me to pick your brain for every last bit of information. I greatly appreciate the extensive sharing of equipment and supplies. Without your assistance, this research would not have been possible. Also, thank you to Rocky, Jon, and Eric for helping with testing on more than one occasion and graciously sharing lab space.

I would like to thank all the people who I've worked with on this NRA, especially Eric Paciano. You have helped me develop ideas and work through issues which arose. I look forward to seeing you succeed in your own testing this fall. Also a thank you to Bryan Blessing and Jon Lichtwardt who aided in the design of the CCR and CFD images.

To Dr. Marshall, thank you for not only supporting my research and further education, but making it possible through this grant. You pose fantastic questions and always make me think carefully about what and why I do what I do.

To Dr. McDonald, you have always been a pleasure. I appreciate having you constantly around to bounce ideas off of. You have made me love posing "what ifs?" in most everything I do. My desire to continue research has been largely encouraged by both you and Dr. Marshall.

To Dr. Jameson my adviser, thank you for challenging me. Your hands off approach pushed me to learn how to develop on my own. Still, when I meandered or needed encouragement, you were there to keep me on track and make sure it got done. I appreciate the life advice you have given me as well and will keep everything in mind as I start in Texas. Thank you.

And most importantly, I would like to thank my family for the constant support and love (most often in cookie form - and hugs). I could not have made it this far without the strong base you instilled in me growing up. Going to Texas will be difficult, but I know I have all your love. 


\section{Table of Contents}

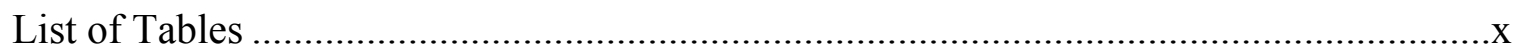

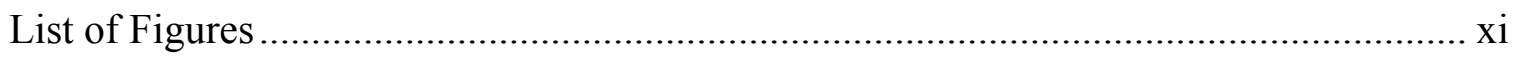

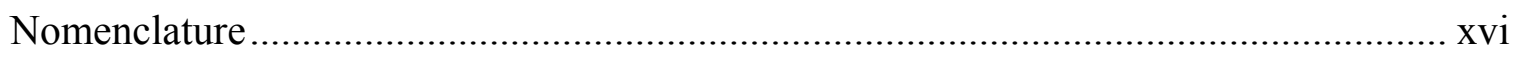

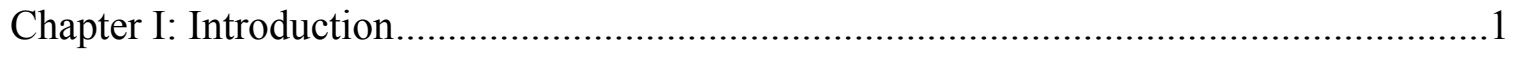

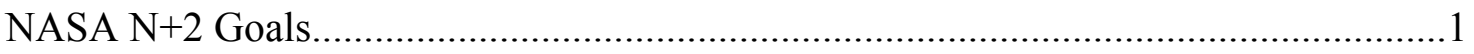

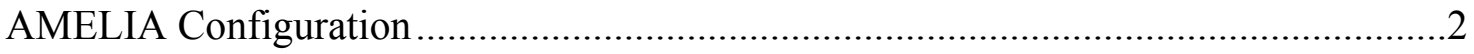

AMELIA Wind Tunnel Model Description .................................................................

AMELIA Wind Tunnel Test ……………………................................................

Chapter II: Uncertainty of Fringe-Imaging Skin Friction Technique ...............................12

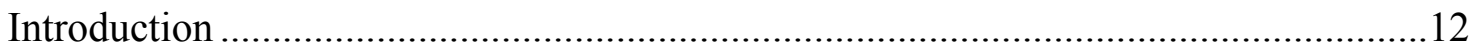

Fringe-Imaging Skin Friction Technique ...................................................................14

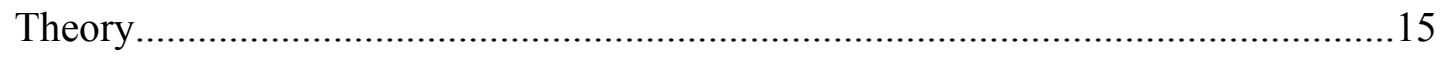

FISF Requirements ..........................................................................................

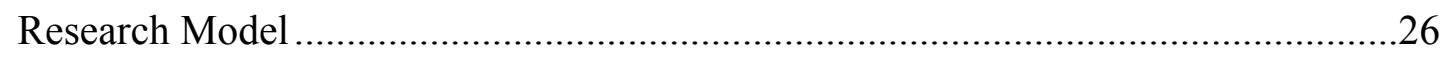

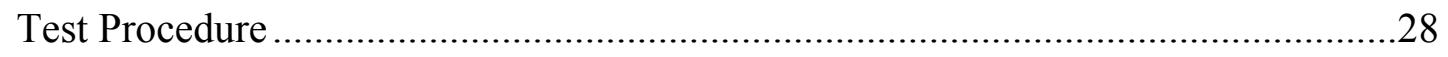

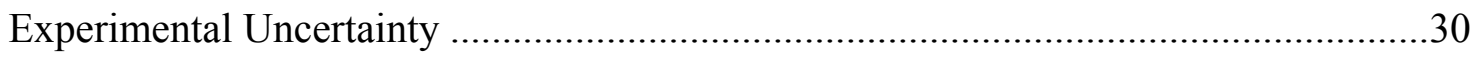

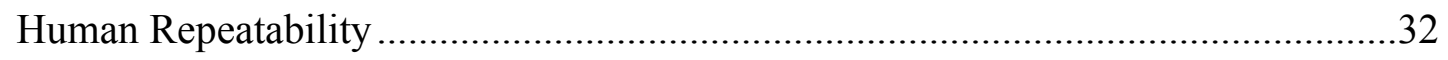

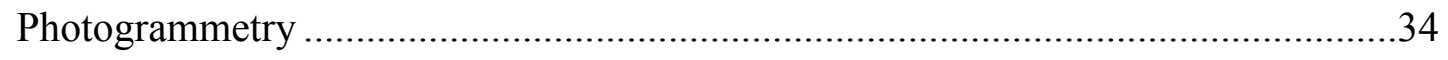

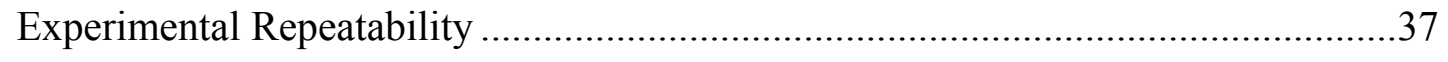

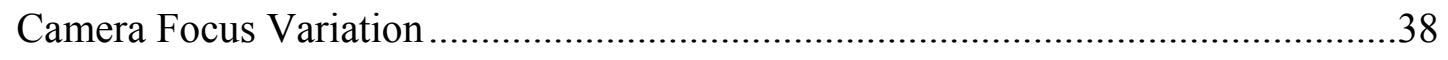

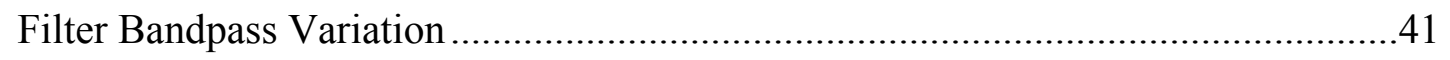

High Dynamic Range and Additional Post Processing ..............................................43 


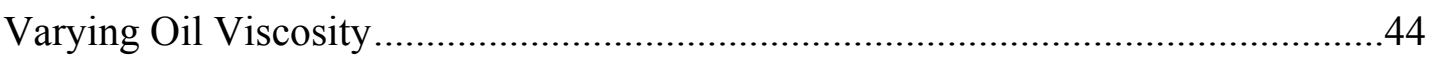

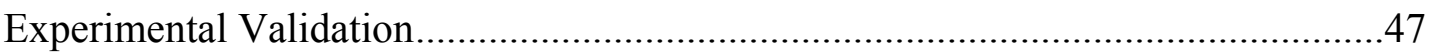

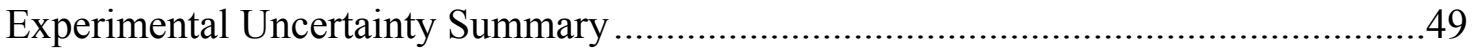

Chapter III: Validation of a Micro Flow Measurement Device..........................................51

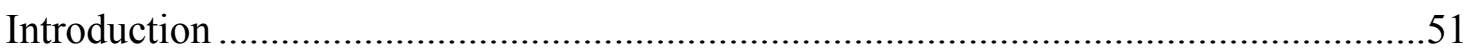

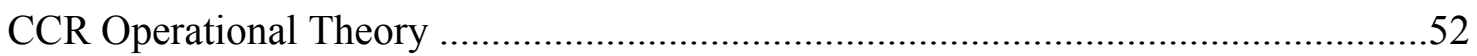

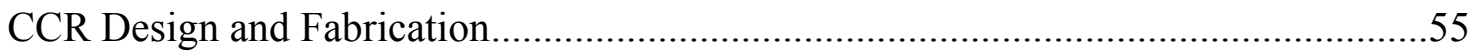

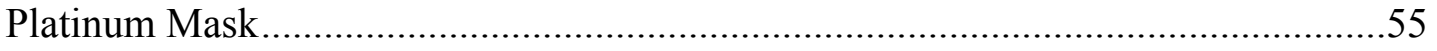

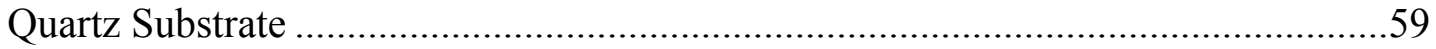

Substrate Base and Flat Plate Insert .....................................................................61

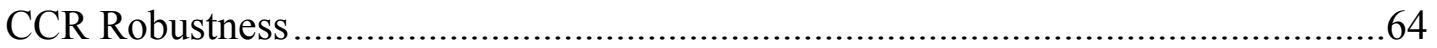

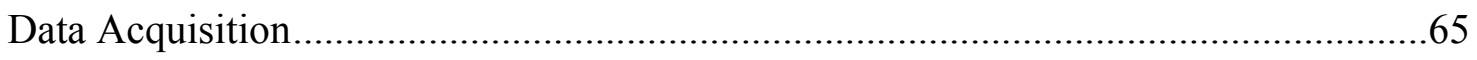

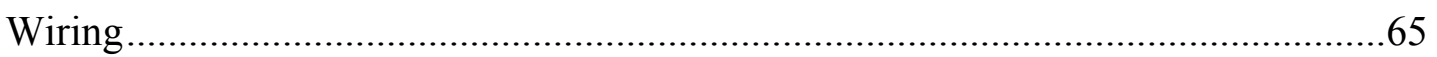

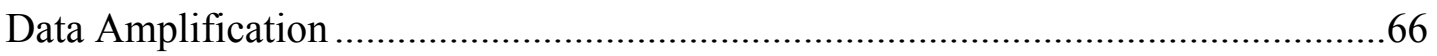

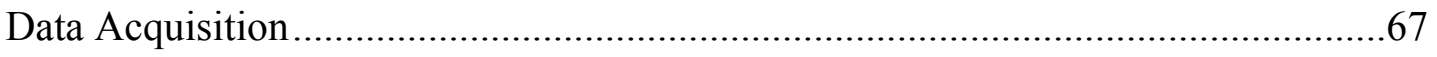

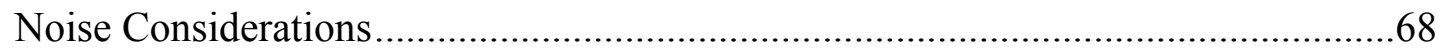

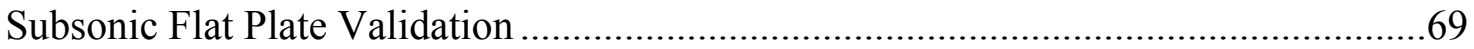

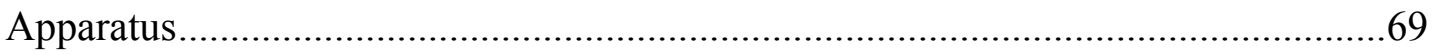

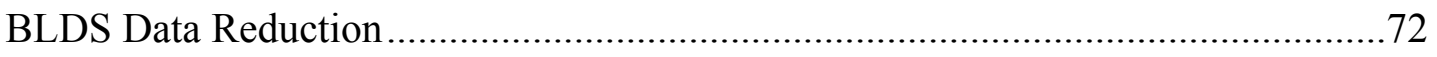

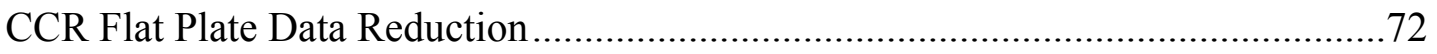

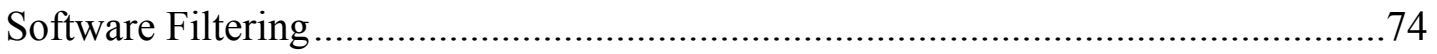

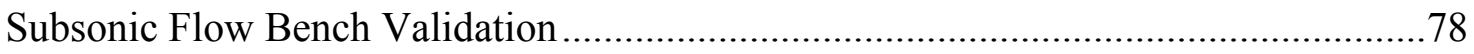

Flow Bench Apparatus ........................................................................................78

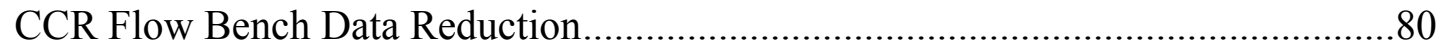

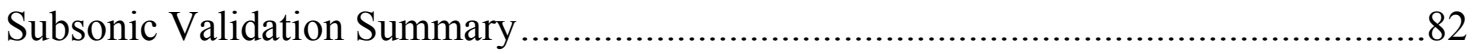


Future Suggestions ......

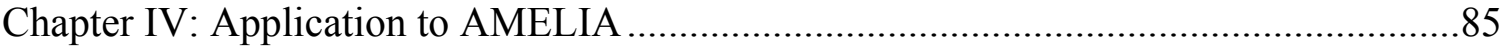

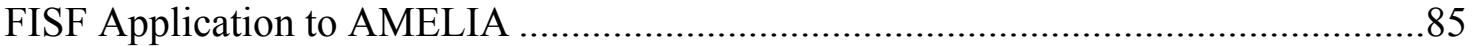

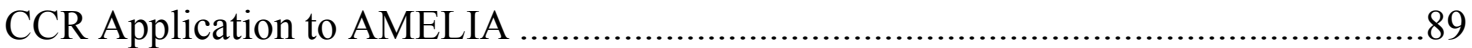

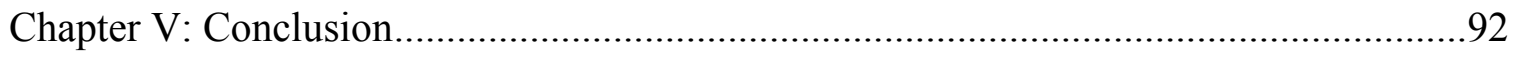

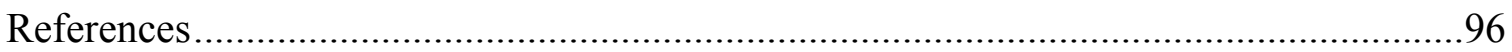




\section{List of Tables}

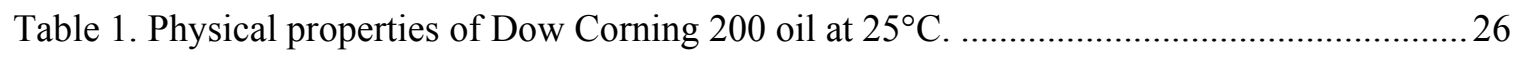

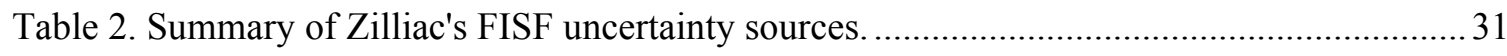

Table 3. Summary of uncertainty due to human repeatability. Point 6 is a directional outlier...... 33

Table 4. Summary of additional error sources investigated.................................................... 50

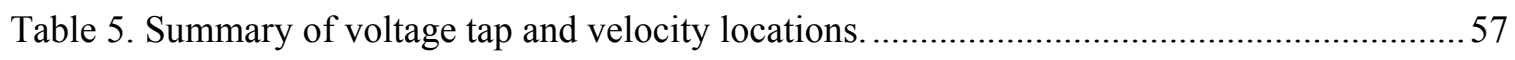

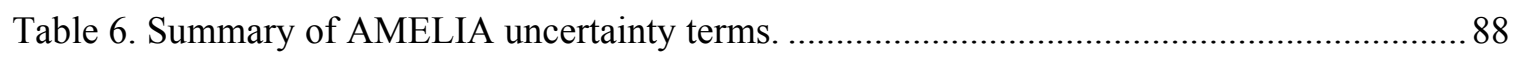




\section{List of Figures}

Figure 1. A rendering of AMELIA shown in three view orientation illustrating the engine over the wing, hybrid wing body, v-tail, and circulation control configuration choices. ................ 3

Figure 2. CC configuration utilized for AMELIA wind tunnel model. Note both leading and trailing edge slots implemented on a supercritical airfoil.

Figure 3. Semi-span layout of AMELIA instrumentation indicating the static pressure port, $\mathrm{CCR}$, and unsteady pressure transducer locations.

Figure 4. Isometric cutaway view of the internal instrumentation within AMELIA, illustrating the high pressure and low pressure air systems within the model.

Figure 5. Isometric view of AMELIA in a section of the NFAC. Farfield microphones and acoustic array are shown.

Figure 6. CFD solution above slot, illustrating the jet boundary layer and shear layer above the flap.

Figure 7. A schematic of the basic FISF setup highlighting the oil flow and fringe pattern on a droplet of oil.

Figure 8. Image of an oil fringe pattern with an axis overlaid. The height is determined from interferometry.

Figure 9. A comparison of fringes on a) black Imron ${ }^{\circledR}$ sample, b) nickel plated wing, and c) stainless steel sample. Each has been polished to a number 2 finish.

Figure 10. A diffuse reflector being lit from below. The dark spot occurs where the camera lens direction is orthogonal to the model surface.

Figure 11. A schematic illustrating that light entering the camera originates from multiple locations, demonstrating the increased lighting difficulty and requirement for model fiducials..... 1

Figure 12. Two photographs of the lighting setup illustrating a) the wing suspended by two tables below and b) a lit white diffuse reflector over the model. The right hole is shown covered with a white cloth.

Figure 13. Image of the fiducial test. The fringe spacing behind the stars (boxed in red) is significantly larger than behind etched fiducial (boxed in yellow), indicating the etched fringes do not trip the flow while the star fiducials do.

Figure 14. Manufacturer emission spectra of the self ballasted mercury vapor lamps 
Figure 15. Comparison of the $35 \mathrm{~nm}$ Barco filter and $6 \mathrm{~nm}$ Semrock filter against the mercury vapor lamp emission spectra. Note the mercury lamp spectra is shown with relative scale on the $y$ axis for comparison purposes.

Figure 16. Comparison of the a) Barco $35 \mathrm{~nm}$ and b) Semrock $6 \mathrm{~nm}$ filter. The aperture, shutter speed, focal distance, and ISO were held constant, with camera held in place with a boom.

Figure 17. A photograph of the nickel plated test article, showing wing blend at far left.

Figure 18. Front view of AMELIA cut on the symmetry plane, illustrating the wing blend to fuselage (shaded green).

Figure 19. Three view of blended wing where the 3D wing blend section is shaded green and the $2 \mathrm{D}$ section is white (all dimensions are in inches).

Figure 20. Image of the 2 foot wing section in $2 \times 2$ foot wind tunnel with access plate visible on far left.

Figure 21. Screenshot of data acquisition software, with green line on the left directly corresponding to fringe intensity versus pixel location graph on the right.

Figure 22. Image illustrating $500 \mathrm{cSt}$ oil fringes on the left compared against large fringe spacing of $100 \mathrm{cSt}$ oil on right.

Figure 23. Image of 2D portion of wing blend with known fringes applied to model surface......36

Figure 24. Contour plot of percent fringe spacing error relative to the location on the wing. The leading edge is particularly poor, while the majority of points are more accurate.

Figure 25. Experimental repeatability of two runs performed eight days apart at a span of 16 inches. A greater variation in data occurred due to interpolating data points at different locations.

Figure 26. High and low limits of the focus distance for various lens focus positions.

Figure 27. Image focus variation from ridge 1 to 8 , with three oil drops shown and one fiducial.

Figure 28. Coefficient of skin friction versus $\mathrm{x} / \mathrm{c}$ location at span location 14 inches. Ridge numbers represent focus distances, with 4 having the best focus and 1 and 8 the worst.

Figure 29. Comparison of the a) $6 \mathrm{~nm}$ Semrock filter illustrating dark ring to the b) $35 \mathrm{~nm}$ Barco filter.

Figure 30. Skin friction coefficient versus $\mathrm{x} / \mathrm{c}$ at a span location of 14 inches for 2 bandpass filters. 
Figure 31. HDR image taken with Semrock $6 \mathrm{~nm}$ bandpass filter with camera reflection clearly visible.

Figure 32. HDR image taken with Barco $35 \mathrm{~nm}$ bandpass filter. Note the high noise region near the center of the image.

Figure 33. Skin friction coefficient data at a span location of 14 inches. The $35 \mathrm{~nm}$ filter without HDR adjust illustrates a consistent variation against the HDR data.

Figure 34. Control experiment for oils of differing viscosity (5 indicating $500 \mathrm{cSt}$ and 1 indicated 100cSt) conducted at $\alpha$ of $0^{\circ}$ and a $q_{\infty}$ of $30.7 \mathrm{psf}$..

Figure 35 . Results of $5.0^{\circ}$ angle of attack test. A sharpie line at $30 \%$ chord differentiates the $100 \mathrm{cSt}$ and $500 \mathrm{cSt}$ oil.

Figure 36. Interpolated oil data points at $x / c$ location of 0.82 illustrating a mean value comparable with Preston's skin friction calibration.

Figure 37. Diagram of the CCR operational methodology with voltage taps shown at A and B locations.

Figure 38. Example of a random function plotted against the same function offset by five seconds.

Figure 39. The function delay of the example random function shown at left, with a clear correlation peak at five seconds.

Figure 40. CFD solution of AMELIA illustrating a plane of contours of Mach number above the flap. The CCR platinum mask is superimposed over the solution. Conditions are at STP, $\alpha$ of $0^{\circ}$, flap $60^{\circ}$, and $M$ of 0.07 .

Figure 41. Schematic of CCR illustrating platinum mask on the quartz substrate and elliptical leading edges.

Figure 42. A schematic (skewed for clarity) of platinum loop resolution, with dimensions in inches.

Figure 43. A photograph of a CCR substrate being shaped. The masking tape protecting the quartz substrate surface is omitted for clarity.

Figure 44. Image of rake base halves separated, illustrating where and how the substrate is held......

Figure 45. Photograph of CCR substrate and base with wiring shown leading out the bottom of the image.

Figure 46. Image of CCR before installation onto the flat plate insert. One o-ring is visible in the lower right side of image which allows for base height adjustment. 
Figure 47. CCR installed onto flat plate insert. Kapton ${ }^{\circledR}$ tape on the non-platinum side is obscuring the lower voltage taps

Figure 48. Electronic schematic of data acquisition setup. Only one voltage differential and six voltage taps are shown for clarity. A total of eight differential amplifiers were required.

Figure 49. Image of the CCR implemented on the flat plate with BLDS in the background.

The flat plate inserts are numbered 1 through 4 , with the CCR on the fourth.

Figure 50. Underside of flat plate illustrating black Mogami cabling, D sub connectors, and grey shielded twisted pair data cable leading out of the tunnel floor.

Figure 51. Boundary Layer Data System with probe locations shown..................................... 71

Figure 52. Second dataset from $170.0 \mathrm{fps}$ run taken at $250 \mathrm{kHz}$ sampling rate........................... 73

Figure 53. Normalization of second dataset from 170.0 fps run taken at $250 \mathrm{kHz}$ sampling rate. Half of each dataset is shown for clarity........................................................................... 73

Figure 54. Cross correlation of normalized second dataset from 170.0 fps run.

Figure 55. Wind tunnel run at $170.0 \mathrm{fps}$ illustrating a) the moving time averaged data and b) the cross correlation of Channel 0 and Channel 7.

Figure 56. Data from Channel 0 of 170.0 fps run with $x$-axis scaled to a) 0.02 seconds and b) 0.001 seconds .76

Figure 57. Discrete Fourier transform of Channel 0 from 170.0 fps run. .77

Figure 58. Data taken while the variable frequency drive is on (noise on left) and off (clean data on right).

Figure 59. Image of flow bench with flat plate insert held up against test section wall.

Figure 60. CCR installed on flat plate insert with vice in background. The arrow indicates

flow direction.

Figure 61. Slope correction in data from flow bench test.

Figure 62. The raw data shown in a) is slope corrected, normalized, and 2,000 point moving time averaged. The correlation in b) shows the velocity of $94.7 \mathrm{fps}$.

Figure 63 . Velocity variation as the number of moving time averaged points increases. 82

Figure 64. Rendering of a possible diffuse reflector for AMELIA. Lighting would be placed below the wing pointing upward to illuminate the reflector surface.

Figure 65. Image illustrating painted fiducials crosshairs and fringes on Imron surface. 87 
Figure 66. Isometric view of the CCR applied to a rendering of the AMELIA wing.

Figure 67. Isometric view of CCR substrate (dark grey), half of CCR base (green), flap (blue) and bottom of wing plenum (red). The platinum mask is shown overlaid on the rake. The light grey circular tube carries the CCR wiring inboard. Two white curved lines represent the flap upper surface relative to the platinum mask. 


\section{Nomenclature}

\section{English Symbols}

$$
\begin{array}{ll}
C & =\text { oil calibration constant } \\
C_{f} & =\text { coefficient of skin friction } \\
f & =\text { camera relative aperture } \\
g & =\text { function } \\
d & =\text { function offset } \\
h & =\text { oil height } \\
M & =\text { Mach number } \\
n & =\text { index of refraction } \\
N & =\text { number of data points } \\
p & =\text { pressure } \\
q & =\text { dynamic pressure } \\
r & =\text { delay function } \\
R & =\text { resistor } \\
t & =\text { time } \\
T & =\text { temperature } \\
u, v, w & =\text { velocity components } \\
V & =\text { voltage } \\
x, y, z & =\text { Cartesian coordinate system } \\
X, Y, Z & =\text { model Cartesian coordinates }
\end{array}
$$

\section{Greek}

$$
\begin{array}{ll}
\alpha & =\text { angle of attack } \\
\Delta s & =\text { fringe spacing } \\
\theta & =\text { skin friction direction } \\
\theta_{r} & =\text { refraction angle } \\
\lambda & =\text { wavelength of light } \\
\mu & =\text { dynamic viscosity } \\
v & =\text { kinematic viscosity } \\
\rho & =\text { density } \\
\tau & =\text { time delay } \\
\tau_{w} & =\text { wall shear stress }
\end{array}
$$

\section{Subscripts}

$$
\begin{array}{ll}
a & =\text { air } \\
o & =\text { oil } \\
s & =\text { voltage source } \\
s & =\text { surface } \\
x, y, z & =\text { Cartesian coordinate system }
\end{array}
$$




\section{Chapter I: Introduction}

With rising environmental and economical concerns, airlines are attempting to both limit the effect airliners have on the environment while maximizing their profitability. While many potential technologies exist, aircraft manufacturers are reluctant to incorporate them into their aircraft due to low Technology Readiness Levels (TRLs). To help bridge this gap between technology and manufacturability, NASA has made NASA Research Announcements (NRA) available under the Subsonic Fixed Wing Project. These NRAs provide grants for universities and private contractors to aid in research tool development.

In October of 2007, David Marshall was awarded an NRA titled "The Integrated Modeling and Verification of Hybrid Wing-Body, Low Noise CESTOL Aircraft.” Phase I was to develop the acoustic and aerodynamic predictive capabilities of a Cruise Efficient, Short Take-Off and Landing (CESTOL) subsonic aircraft and to complete a preliminary design of a wind tunnel model and test which would serve to validate newly developed codes. Upon receiving Phase II, continued progress is being completed towards predictive code development. A large scale wind tunnel model is being built and will be tested with the purpose to aid in the final validation of the predictive capabilities. The wind tunnel test will also create an open collection of data for future code validation efforts. The project has been titled the Advanced Model for Extreme Lift and Improved Aeroacoustics (AMELIA). Currently, the NRA is in year three of four with the wind tunnel test slated for the summer of 2011. A more complete summary of the project is available in Marshall. ${ }^{1}$

\section{NASA N+2 Goals}

NASA developed goals for future aircraft noise, $\mathrm{NO}_{\mathrm{x}}$ emissions, fuel burn, and field length. These goals are defined by $\mathrm{N}+1, \mathrm{~N}+2$, and $\mathrm{N}+3$ whose timeframes range from 2015, 2020, and 2025, respectively. The $\mathrm{N}+2$ goals are defined as a $-42 \mathrm{~dB}$ (cumulative 
below Stage 4), $-75 \%$ landing and takeoff $\mathrm{NO}_{\mathrm{x}}$ emissions (below Committee on Aviation Environmental Protection (CAEP) 6), $-40 \%$ aircraft fuel burn, and $-50 \%$ reduction in field length. TRLs of 4-6 were the main focus of this study, with the aim of implementing an aircraft with similar design and technologies by 2020 .

\section{AMELIA Configuration}

The AMELIA design stemmed specifically from $\mathrm{N}+2$ technologies. A three view of the resulting design is shown in Figure 1. The main goal of the NRA was to make progress towards improved noise and field length performance; these were the focal points of the design configuration. Improving field length performance increases the number of airports out of which an aircraft can operate. Larger aircraft easily operate from locations which are already equipped to handle longer field requirements. However, a burgeoning market which airlines are more interested in are regional flights. These are short, direct, hub to spoke flights. Most of the flight time is spent during climb and descent. With this in mind, the first constraint placed on AMELIA was a 100 passenger, regional aircraft. To improve field performance, various high lift devices were investigated. The final decision was the implementation of a circulation control (CC) wing. The goal of utilizing CC is to drastically increase lift with a limited drag rise during takeoff or landing. This is achieved by blowing high speed air tangentially from slots onto the wing surface at the leading and trailing edges of the wing. An example airfoil section from AMELIA is illustrated in Figure 2. 

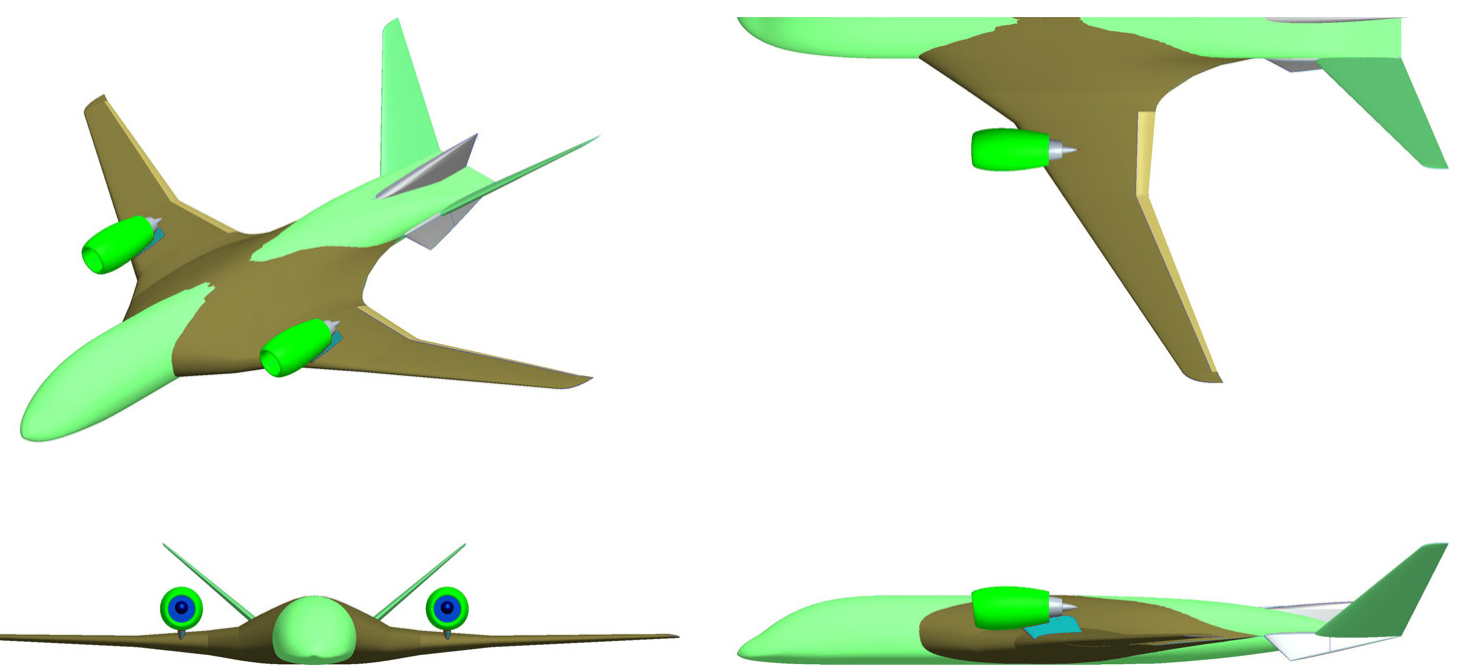

Figure 1. A rendering of AMELIA shown in three view orientation illustrating the engine over the wing, hybrid wing body, v-tail, and circulation control configuration choices. ${ }^{2}$

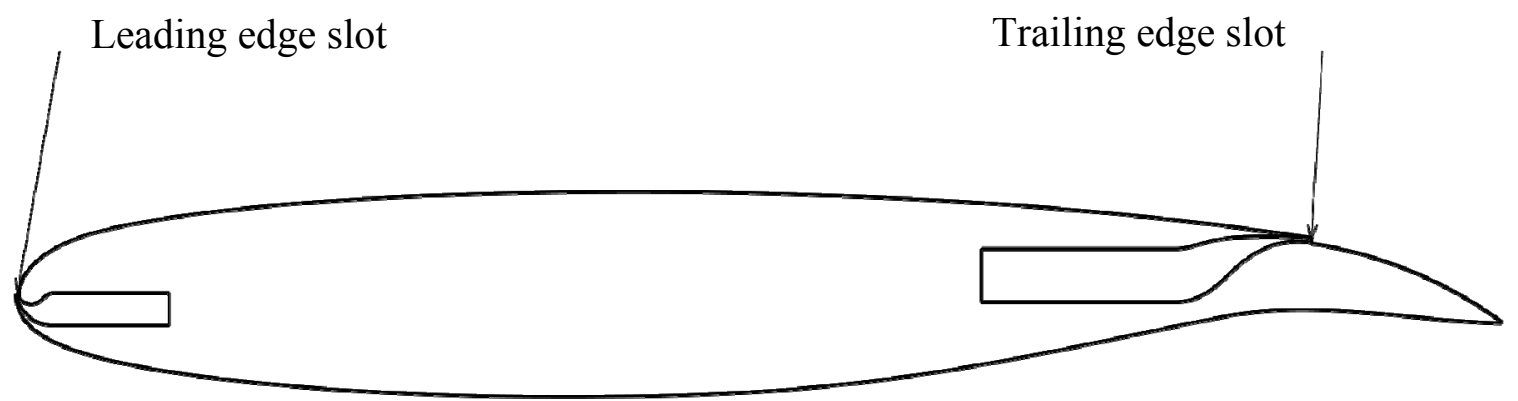

Figure 2. CC configuration utilized for AMELIA wind tunnel model. Note both leading and trailing edge slots implemented on a supercritical airfoil. ${ }^{2}$

A CC configuration has numerous advantages. By utilizing a design represented by the airfoil in Figure 2, there is a vast reduction in mechanical parts compared to a traditional double/triple slotted flap configuration with leading edge slats. The moving parts reduce to the dual radius flap which has a single pivot point and covers for the slots during cruise portions of the mission. A large portion of the noise from aircraft today comes from high energy vortices being shed from the flap configuration. In theory, CC reduces the noise by simplifying the high lift components on the wing. However, the slot velocity is near sonic, causing high noise levels as well. $\mathrm{CC}$ also has the disadvantage of requiring bleed air from the engines which can drastically reduce engine performance. 
With many engines going towards bleedless, all electric designs, electric compressors could be utilized. Dedicated gas generators are another option to supply the slot airflow. While CC technology has a high TRL having successfully been implemented on an A- $6^{3}$ and BD-4 general aviation aircraft, ${ }^{4}$ the technology is difficult to model and further research in this area is highly desirable.

To continue to reduce community noise, the aircraft engines were placed over the wing to shield turbine and exhaust noise. Georgia Tech Research Institute (GTRI) completed a study ${ }^{5,6}$ to determine the best location aerodynamically and acoustically for the engine. From these results, an engine location was selected to be implemented on the AMELIA model. With the engines placed over the wings, a V-tail configuration was chosen to avoid jet wash over the horizontal control surfaces. A secondary advantage of the engine placement combined with the $\mathrm{CC}$ wing is the ability to entrain the engine flow. $^{7}$ The significant turning from the $\mathrm{CC}$ has been shown in Computation Fluid Dynamics (CFD) solutions to entrain the engine flow downward, thereby further increasing the aircraft lift. The AMELIA test will help validate this phenomena's occurrence.

Lastly, a hybrid-wing body was applied to AMELIA. While not focusing on cruise efficiency, the hybrid-wing body possibly improves the lift to drag ratio by improving wing-body aerodynamics. As an additional configuration benefit, the extra wing volume encloses the landing gear, removing the need for landing gear fairings, further reducing drag. Additional storage space for baggage or subsystems is also obtained by utilizing the hybrid-wing, allowing the fuselage design to stray from the traditional double bubble or cylindrical configurations. 


\section{AMELIA Wind Tunnel Model Description}

Due to the nature of the technologies being tested, the wind tunnel model design required for successful testing is quite complicated. AMELIA is being constructed by Patersonlabs, Inc., located in Kent, Washington. The model configuration has four flap deflections, two engine locations, variable slot and engine mass flow rates, an Imron ${ }^{\circledR}$ coating, and various Kulite and static pressure port locations. Turbine Propulsion Simulators (TPS) units were chosen over a flow through nacelle or clean configuration to more accurately model turbine noise and effects of engine flow entrainment.

The flaps are removable with deflections at $0^{\circ}, 30^{\circ}, 60^{\circ}$, and $80^{\circ}$. From research completed by Englar ${ }^{5}$ and Gaeta, ${ }^{6}$ two ideal engine pylon heights were chosen for further investigation. A summary of the model instrumentation is shown in Figure 3. In total, there are 280 static pressure ports at five chordwise and one spanwise location. There are three unsteady pressure transducers on the wing blend at passenger level, three inline behind the engine, and two within the nacelle. There is a slot in the flap which allows for the application of a boundary layer rake.

The high and low pressure air system is illustrated in Figure 4. High pressure air is required to run the TPS units. The high pressure air enters through an eight inch flow through balance and is controlled with two, independent actuators located near the nose of the model. The low pressure air system passes through two bellows before entering a plenum. From the plenum, the flow is led to two leading edge and two trailing edge plenums for the slots. Again, each slot mass flow rate is independently controlled. The high and low pressure air system is mirrored on the right side of the model. 


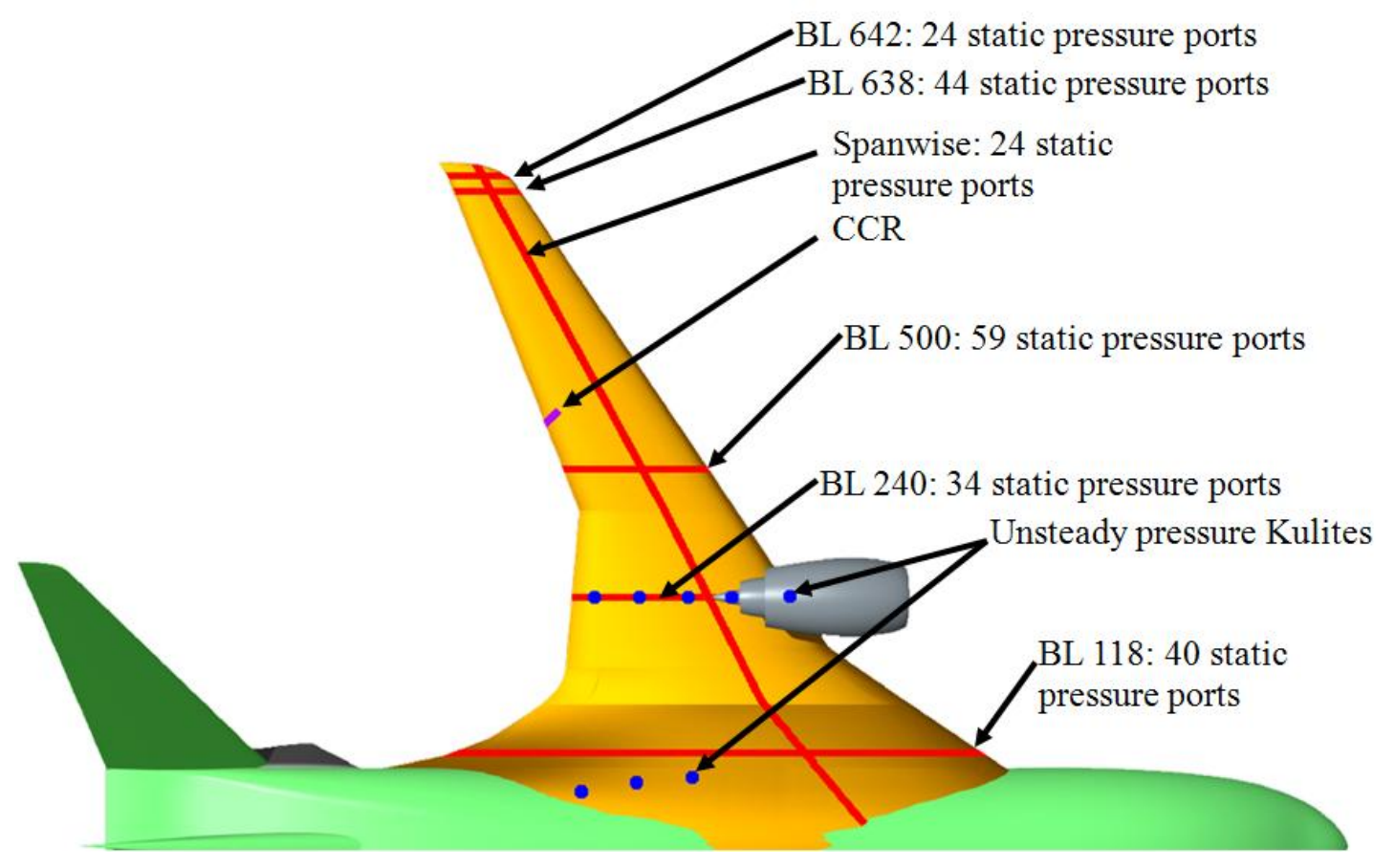

Figure 3. Semi-span layout of AMELIA instrumentation indicating the static pressure port, CCR, and unsteady pressure transducer locations. ${ }^{8}$

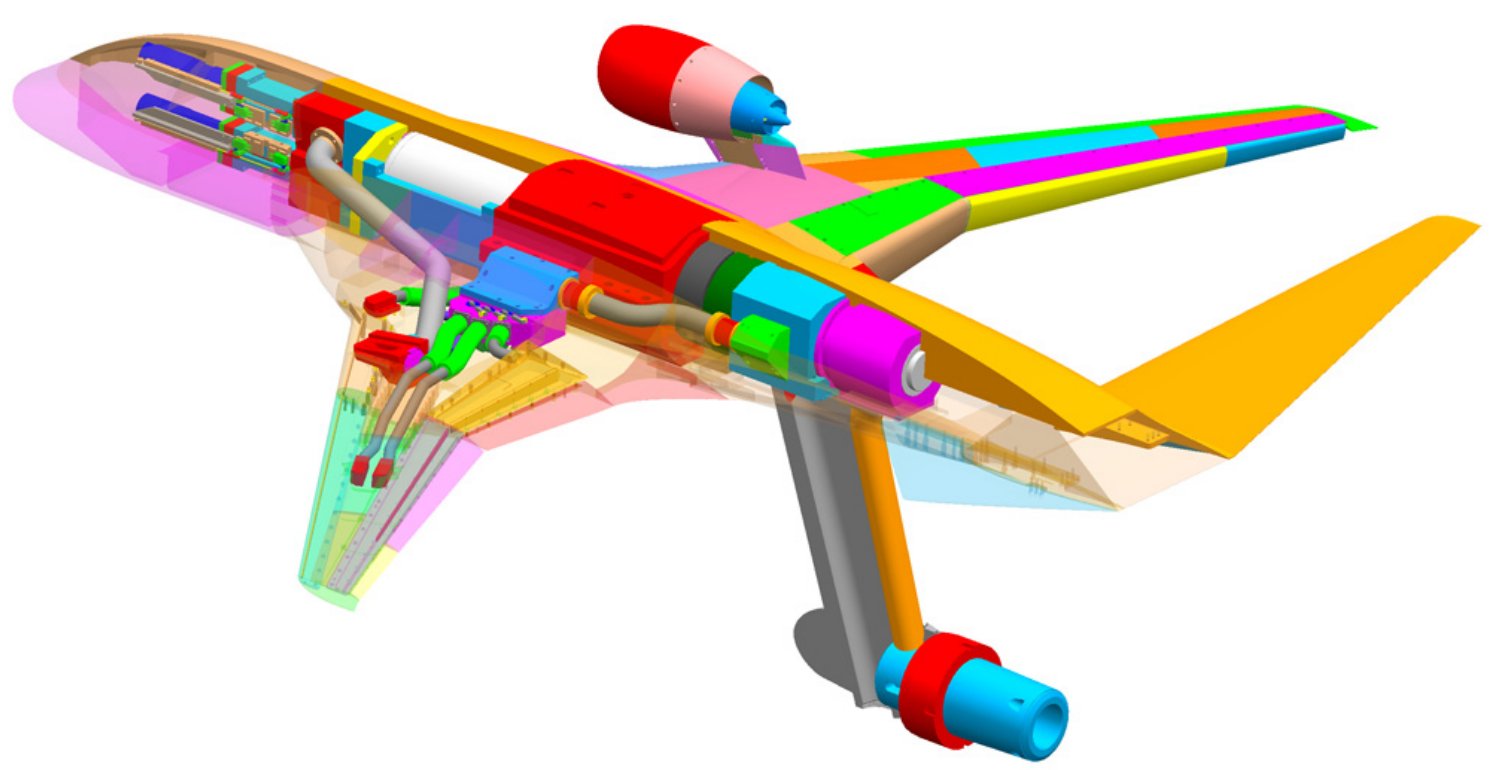

Figure 4. Isometric cutaway view of the internal instrumentation within AMELIA, illustrating the high pressure and low pressure air systems within the model. ${ }^{8}$ 


\section{AMELIA Wind Tunnel Test}

Since the NRA's goal is to create better predictive capabilities, a dataset is needed to validate the improved capabilities. There is a wide range of $2 \mathrm{D} \mathrm{CC}$ data in existence

today. ${ }^{9-14}$ With the advent of new measurement techniques such as PIV, large regions of the flow field can be measured and compared to 2D CFD solutions. ${ }^{14,15}$ These techniques have been implemented to create databases for $2 \mathrm{D} \mathrm{CC}$ comparisons. While progress is being made towards $2 \mathrm{D} \mathrm{CC}$ validation efforts, less has been made in $3 \mathrm{D}$ validation. Collins ${ }^{16}$ and Lui ${ }^{17}$ completed a 3D test of a hybrid wing body model with blown flaps in 2008. Force, moment, static pressure ports, Pressure Sensitive Paint (PSP), and wake measurements were completed yielding a large dataset for validation efforts. However, still lacking were skin friction and acoustic measurements. Beyond that, no full aircraft, aerodynamic and acoustic open source testing has been completed to date. AMELIA offers a unique opportunity to have a full dataset for validation efforts.

As with any wind tunnel test, care must be taken to determine what measurements are to be made. A list of all the desired measurements for a complete CFD validation was compiled. Due to facility and monetary limitations, there is a range of feasible measurements which dictates the final test plan. It was decided that force, moment, pressure distributions, surface unsteady pressure measurements, and farfield unsteady pressure measurements were required for the test to be successful. Highly desirable measurements included global skin friction, flap velocity profiles, PIV, wake survey, phased microphone array, and PSP. For a complete validation effort, CFD needs a minimum of pressure distributions and skin friction direction and magnitude. 
Measurement capabilities vary greatly depending on wind tunnel. Since the test has goals for validation in both aerodynamic and acoustic regimes, it is important to utilize a tunnel with an acoustic liner. Most wind tunnels have temporary acoustic treatments that are applied specifically for acoustic measurements. Usually these treatments result in decreased flow quality and changes to the test section size. For example, the Langley 14 foot by 22 foot tunnel is closed circuit for aerodynamic measurements and open jet with an acoustic liner for acoustic measurements. This is undesirable since aerodynamic and acoustic measurements become difficult to link in a useful way. A second requirement was a tunnel sufficiently large to make wall effects negligible. Utilizing preliminary CFD solutions, ${ }^{7}$ it was determined that a minimum distance of one span is required above and below the model. The model was specified to have a minimum span of eight feet in the NRA. To avoid slot scaling issues a 10 foot span was chosen. Lastly, the high pressure air requirements are large due to the blown leading and trailing edge slots and TPS units. These requirements severely limit the wind tunnel availability. However, the National Full-Scale Aerodynamics Complex (NFAC) 40 by 80 foot tunnel proved to meet the above requirements. With the model suspended in the center of the tunnel, there are two spans above and below the model. The tunnel has a 3.5 foot thick acoustic liner applied to the walls, allowing for very low to ultrasonic frequencies to be measured while maintaining good flow quality. ${ }^{18}$ A rendering of AMELIA in the NFAC is shown in Figure 5. 


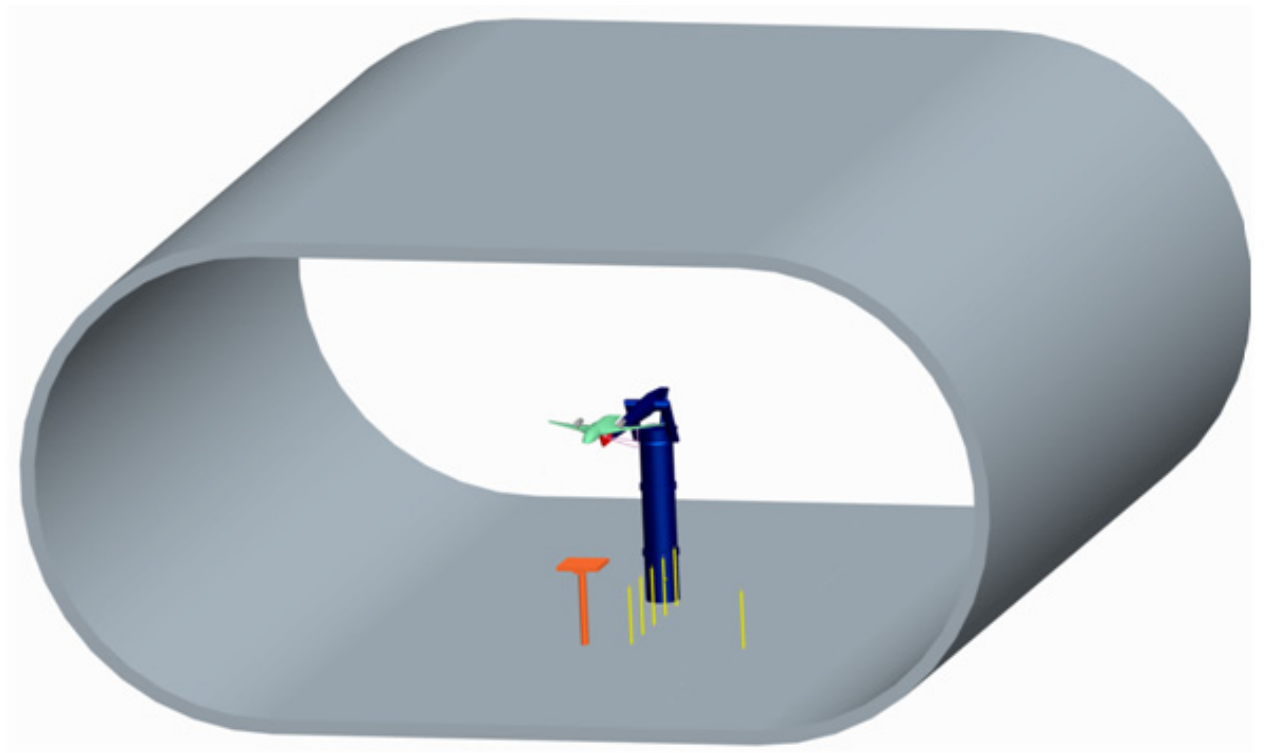

Figure 5. Isometric view of AMELIA in a section of the NFAC. Farfield microphones and acoustic array are shown. ${ }^{8}$

Static pressure ports yield sufficient pressure data for CFD validation so PSP is not vital. PSP also requires visual access to the model. The NFAC is not equipped with many viewing locations and the distance between the model and the camera is at a minimum 20 feet, making visual access difficult. Similar issues arise with PIV. Currently, a rotorcraft test is making PIV wake measurements in the NFAC. In correspondence with Alan Wadcock, a NASA employ who implemented PIV for the rotorcraft test, ${ }^{19}$ applying PIV in the NFAC would be a costly and time consuming endeavor. Due to budget constraints, PIV was deemed beyond the project scope. A wake survey with a traversing system would be a second option to PIV. However, many of the old traversing systems have been misplaced, requiring new systems to be developed. A system designed to traverse from 10 to 20 feet while maintaining accurate location would be extremely difficult to manufacture. Between the system and the 7-hole probe required, the cost would be prohibitively large. The phased microphone array hardware was available for use, reducing the cost of utilizing the equipment. ${ }^{20}$ It will therefore be employed. It was 
decided that global skin friction measurements were a necessity for the validation effort, as little skin friction data exists for CC airfoils. The Fringe-Imaging Skin Friction (FISF) technique will be applied on AMELIA, with further discussion in Chapter II. Lastly, slot jet and shear layer measurements above the flap were desired. These measurements will be made with micro boundary layer measurement device known as a Cross Correlation Rake (CCR), discussed further in Chapter III.

Because FISF measurements require long tunnel runs and yield one dataset per tunnel run, only 10 test points will be investigated. Since the CCR is an invasive measurement device, it will only be utilized on these 10 test points. A phased array, $30^{\circ}$ sideline, and polar microphone measurements will be implemented throughout the test. Following this, the AMELIA test will run as a standard wind tunnel test, with $\alpha$ sweeps from $-5^{\circ}$ to $22^{\circ}$ by $3^{\circ}$ increments, $\beta$ sweeps from $0^{\circ}$ to $20^{\circ}$ by $4^{\circ}$ increments, two engine locations, four flap deflections, three Reynolds numbers, and three CC slot mass flow rates. During the entire test, static pressure port, force, and moment data will be taken. Jameson ${ }^{8}$ further outlines the AMELIA test.

Skin friction and shear layer velocity profiles are important measurements for this particular test since the goal is CFD validation. Local measurements (static pressure and skin friction) are of much greater use than integral measurements (force and moment). Lift and drag can be somewhat easy for CFD to match with reasonable accuracy due to cancelling inaccuracies which can occur during the integration of pressures and skin friction. Therefore, local measurements provide a more realistic dataset to validate CFD. Skin friction is somewhat unique in that global measurements are only recently becoming 
common place in large scale tests. ${ }^{21,22}$ The FISF application to AMELIA will yield an extremely useful dataset to the CFD community.

One particular area that turbulence models fail to accurately predict is shear layers. $^{23,24}$ Over the flap, there is a high speed jet mixing with the high speed jet layer from the leading edge slot (which has decelerated at this location). This flow field is illustrated in a CFD solution in Figure 6 below. Measurements in the jet boundary layer and shear layer are desired for CFD validation. In some cases, separation can occur, which would be of great importance to CFD. Measurements in such a small, localized location are difficult to make. A new device was developed by NASA Glenn and will be implemented on the AMELIA flap with the goal of making these measurements.

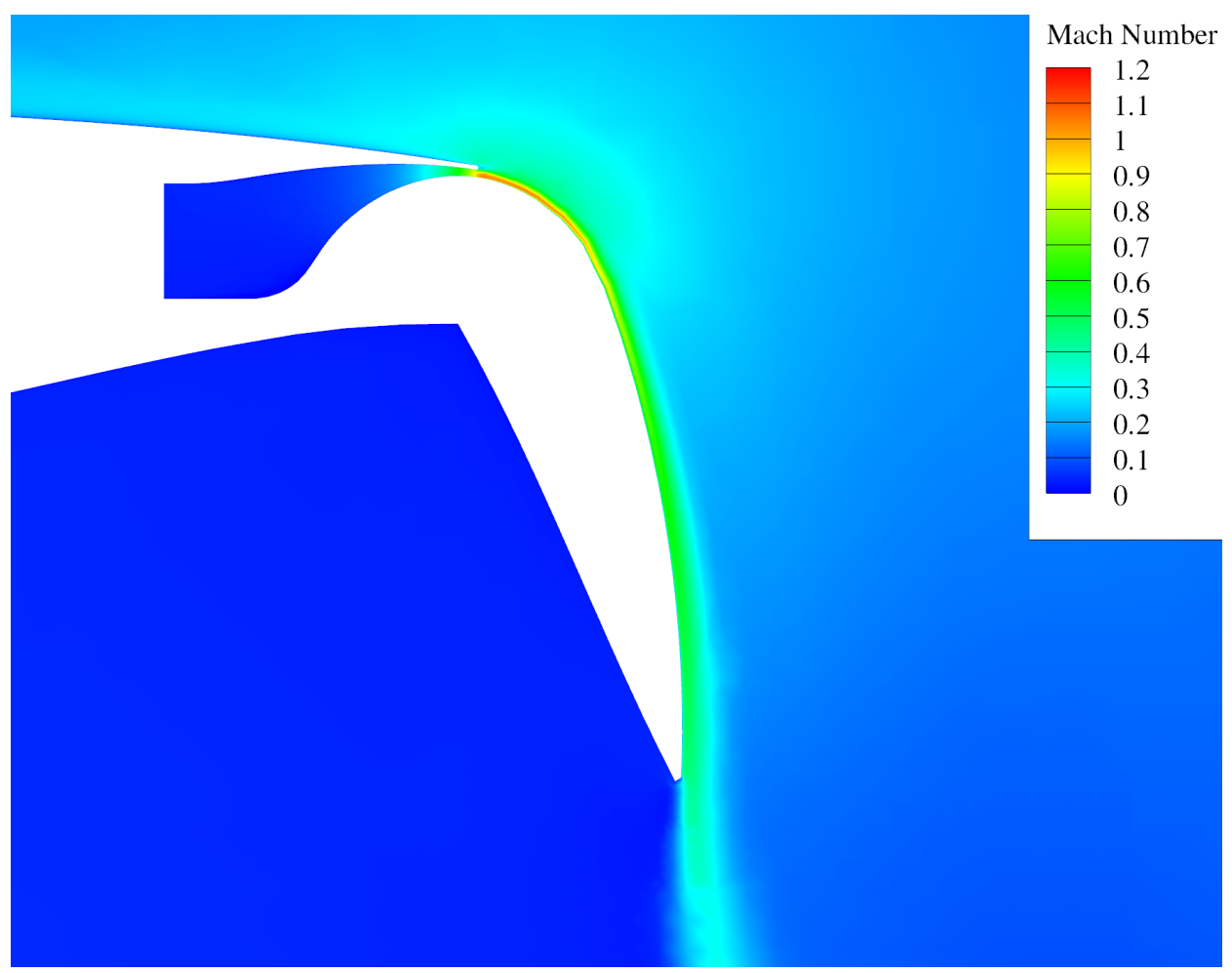

Figure 6. CFD solution above slot, illustrating the jet boundary layer and shear layer above the flap.

${ }^{*}$ Lichtwardt, J. A., Private communication, June 2010. 


\section{Chapter II: Uncertainty of Fringe-Imaging Skin Friction Technique}

\section{Introduction}

There are several methods of measuring skin friction including Preston tubes, Stanton gauges, hot films, Microelectromechanical Systems (MEMS), liquid crystals, and FringeImaging Skin Friction (FISF) techniques, each having their own advantages and disadvantages. ${ }^{25-29}$ Preston tubes and Stanton tubes are classical devices proven to yield excellent results for 2D, developed boundary layers. Probes must be carefully positioned to avoid adverse changes in flow features, limiting global measurement capability. Hot films are useful in detecting transition; however, the extension of this technique to skin friction magnitude is challenging requiring calibration with a Preston tube. ${ }^{30}$ MEMS have the unique capability to measure fluctuations in the shear stress. The integration of multiple MEMS into a model would prove impractical and monetarily prohibitive for most applications. Liquid crystals are most effective on flat surfaces and offer global direction and magnitude measurements. Difficulties arise due to visual access, accuracies, and calibration. ${ }^{31}$ Due to the high curvature of the model and visual requirements, liquid crystals were deemed inappropriate. The FISF technique is non-intrusive and allows for global skin friction direction and magnitude measurements. FISF is a low cost method, but can rapidly become expensive depending on the price of tunnel time and the desired dataset. The technique yields results with accuracy ranging from $\pm 2.8 \%$ to $\pm 13.6 \%$ for skin friction magnitude and $\pm 1^{\circ}$ for skin friction vector direction. ${ }^{32}$ To date, only liquid crystals and FISF offer global measurements. A surface stress sensitive film is currently 
under development and shows promise as a future global skin friction magnitude and direction measurement technique. ${ }^{33}$

The FISF technique is a proven method and has been successfully applied to a wide range of tests, from subsonic channel flows to supersonic laminar and turbulent flows. ${ }^{32,34}$ Large scale wind tunnels have additional testing difficulties due to model access, lighting requirements, visual access, and long tunnel transients. Driver ${ }^{35}$ investigates these issues and summarizes various FISF tests in large wind tunnels. These difficulties must be addressed in the application of FISF in the AMELIA test. In preparation for the test, a two foot wing section was manufactured to further explore uncertainties related to the FISF technique. While the majority of skin friction uncertainties have been well described by Zilliac ${ }^{32}$ and Naughton, ${ }^{36}$ developments in digital photography and concerns with the AMELIA test have fostered interest in a more detailed uncertainty analysis.

Skin friction magnitude and direction will be measured on a two foot wing blend section with the goal of better characterizing uncertainties. The results will be directly applied to the AMELIA test, further improving the measurement quality. This investigation will address the uncertainties listed below:

- Human repeatability in fringe spacing measurement and direction

- Photogrammetry calibration due to fiducial misplacement and/or model inaccuracies

- Experimental repeatability

- Varying image focus

- Filter variation and effect on light wavelength measurement 
- Utilization of High Dynamic Range (HDR) images and additional postprocessing techniques

- Application of oils of varying viscosity

- Validation against Preston tube with full error propagation

\section{Fringe-Imaging Skin Friction Technique}

The FISF technique was developed by Monson, et al. ${ }^{37}$ The essential theory behind the technique is that a simple expression can relate the thickness of the oil at a single point to the skin friction magnitude and direction. The oil thickness is measured via interferometry, illustrated in Figure 7. Data reduction is completed with CXWIN5G, a PC application developed by Gregory Zilliac. ${ }^{38}$ The technique itself has recently become a popular method for measuring skin friction on large scale wind tunnel models. ${ }^{39}$

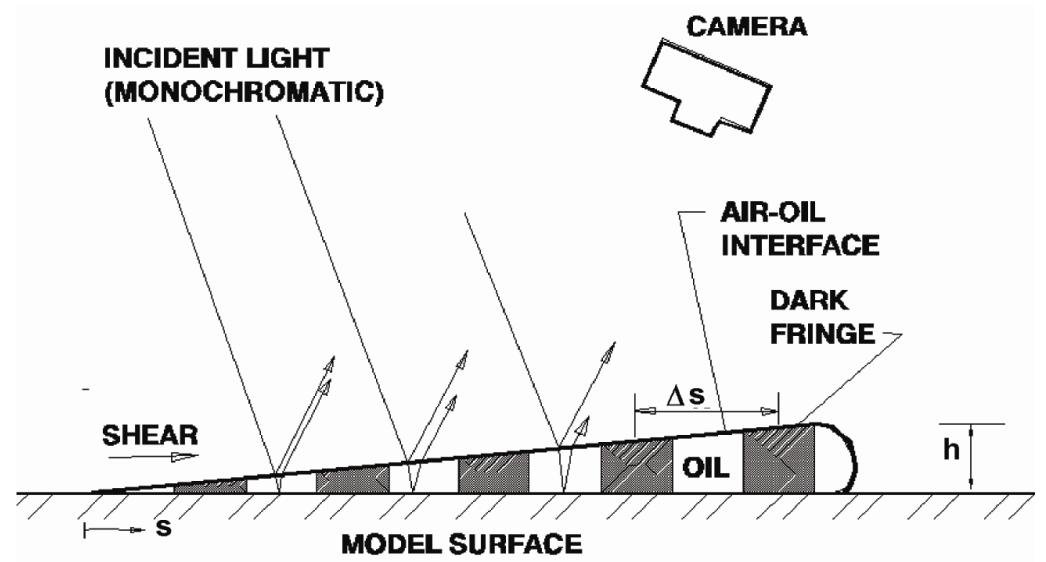

Figure 7. A schematic of the basic FISF setup highlighting the oil flow and fringe pattern on a droplet of oil. ${ }^{39}$

The FISF process is as follows: A drop of silicone oil of known viscosity is placed on the model surface. Once the oil is applied to the surface, the air flow is started, causing the oil to spread and thin. The air continues to flow for a given time, continually thinning the oil. When sufficient time has elapsed (5 to 20 minutes, depending on oil viscosity and 
desired fringe spacing), the air flow is turned off. A quasi-monochromatic light source is then indirectly applied to the surface by use of a large diffuse reflector. The light from the reflector is reflected from the both the surface of the model and oil, separated by the thickness of the oil, shown in Figure 7. The assumed linearly varying oil height creates an interference pattern from the constructive and destructive interference between the light reflecting from the model and oil surface. This interference pattern can be utilized to measure the fringe spacing which is correlated to the skin friction.

\section{Theory}

Squire ${ }^{40}$ derived Equation (1) by applying a control volume approach to an oil film on a surface with an external shear (air flow) applied as the upper boundary condition.

$$
\frac{\partial h}{\partial t}=\frac{1}{\mu_{o}}\left\{\frac{1}{3} \frac{\partial}{\partial x}\left(h^{3} \frac{\partial p}{\partial x}\right)-\frac{\mu_{a}}{2} \frac{\partial}{\partial x}\left[h^{2}\left(\frac{\partial u_{a}}{\partial y}\right)_{y=h}\right]+\frac{1}{3} \frac{\partial}{\partial z}\left(h^{3} \frac{\partial p}{\partial z}\right)-\frac{\mu_{a}}{2} \frac{\partial}{\partial z}\left[h^{2}\left(\frac{\partial w_{a}}{\partial y}\right)_{y=h}\right]\right\}
$$

where $h$ is the height of the oil, $t$ is time which shear has been applied to the oil, $\mu_{o}$ is the oil viscosity, $\mu_{a}$ is the air viscosity, $u_{a}$ and $w_{a}$ are the velocity components of air, and $p$ is pressure. By assuming the oil flow is $2 \mathrm{D}$, incompressible, and slow and that gravity, pressure gradients, and shear stress gradients are negligible, Equation (1) can be reduced to

$$
\frac{\partial h}{\partial t}=\frac{1}{\mu_{o}}\left\{-\frac{\mu_{a}}{2} \frac{\partial}{\partial x}\left[h^{2}\left(\frac{\partial u_{a}}{\partial y}\right)_{y=h}\right]-\frac{\mu_{a}}{2} \frac{\partial}{\partial z}\left[h^{2}\left(\frac{\partial w_{a}}{\partial y}\right)_{y=h}\right]\right\}
$$

If the air velocity is much larger than the oil velocity, then $\left(\partial u_{a} / \partial y\right)_{y=h}$ and $\left(\partial w_{a} / \partial y\right)_{y=h}$ are approximately $\tau_{x y} / \mu_{a}$ and $\tau_{y z} / \mu_{a}$ where $\tau_{x y}$ and $\tau_{y z}$ are the wall shear forces, reducing Equation (2) to

$$
\frac{\partial h}{\partial t}=\frac{1}{\mu_{o}}\left\{-\frac{\mu_{a}}{2} \frac{\partial}{\partial x}\left[h^{2} \frac{\tau_{x y}}{\mu_{a}}\right]-\frac{\mu_{a}}{2} \frac{\partial}{\partial z}\left[h^{2} \frac{\tau_{y z}}{\mu_{a}}\right]\right\}
$$


By aligning the skin friction with the $x$ axis, the differential equation becomes

$$
\frac{\partial h}{\partial t}=-\frac{1}{\mu_{o}} \frac{\partial}{\partial x}\left[\frac{h^{2}}{2} \tau_{w}\right]
$$

A linearized solution can be found by assuming that $\tau_{w}$, the wall shear stress, is constant in both the $x$ direction and in time. At a time of zero, the oil height is assumed to be infinite. The result is Equation (5), shown below

$$
\tau_{w}=\frac{\mu_{o} x}{h t}
$$

where the variable $h$ is the oil height at some position $x$ along an oil drop. Utilizing interferometry, the height of the oil can be determined from the light wavelength and oil index of refraction, $n_{0}$. This is illustrated in Figure 8.

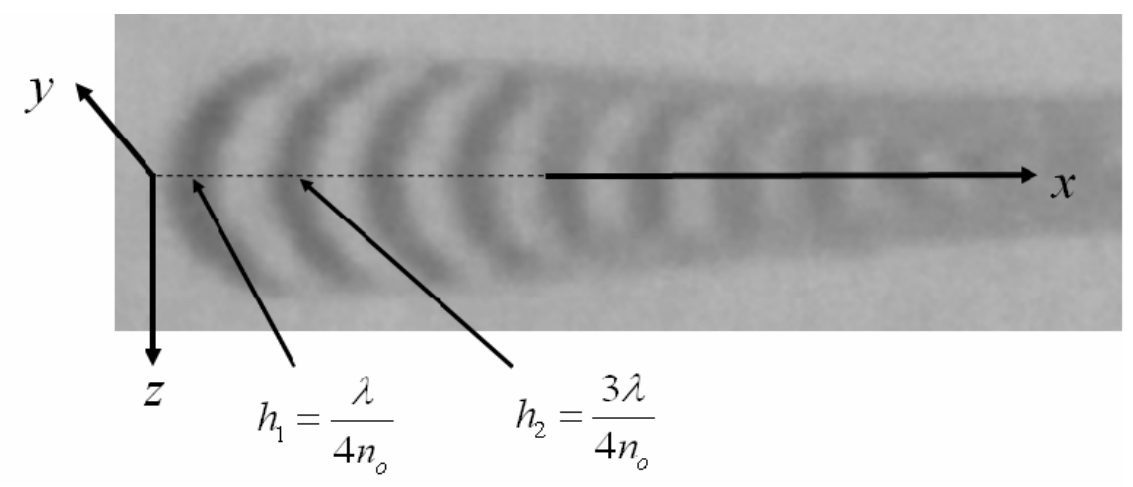

Figure 8. Image of an oil fringe pattern with an axis overlaid. The height is determined from interferometry.

By substituting the heights, the $x$ location for each fringe can be determined. Equation (6) results by subtracting the two fringe locations

$$
C_{f}=\frac{\tau_{w}}{q_{\infty}}=\frac{2 n_{o} \mu_{o} \Delta s}{q_{\infty} \lambda t} \cos \theta_{r}
$$

where $C_{f}$ is the coefficient of skin friction, $q_{\infty}$ is the freestream dynamic pressure, $n_{o}$ is the oil index of refraction, $\mu_{o}$ is the oil viscosity, $\Delta s$ is the fringe spacing, $\lambda$ is the wavelength of the light source, $t$ is the duration of time the oil flow was exposed to air flow, and $\theta_{r}$ is 
the light refraction angle through the air-oil interface. ${ }^{38}$ The fringe spacing distance directly relates to the thickness of the oil, which is proportional to the skin friction. If the fringe spacing is known, then the skin friction can be calculated. When applied to the AMELIA test, care must be taken in the assumption that pressure and shear stress gradients are zero, especially near the circulation control slots.

Monson et al. ${ }^{37}$ originally introduced FISF as an extension of work completed by $T^{T a n n e r}{ }^{41}$ on a photographic version of the laser interferometer skin-friction technique. Since Monson, further developments have been made in the image processing, improving the accuracy of the fringe spacing measurements and the ease of technique implementation.

\section{FISF Requirements}

\section{Surface Finish}

In order to successfully apply the FISF technique, the fringes need to be clearly visible. Fringe visibility is based upon the surface finish and material of the model and is a maximum when the air-oil and specular oil-surface are reflections are matched, and the remaining light is excluded. An ideal surface is extremely smooth, has consistent and durable optical properties, and an index of refraction, $n_{s}$, of 2.0. Zilliac completed a study to determine the ideal material for FISF application. ${ }^{32}$ The best fringes appeared on high flint content SF11 glass manufactured by Schott Glass, with an $n_{s}$ of 1.78 . This is obviously an impractical material for a wind tunnel model. The ideal surface finish is mirror like, with acceptable substitutes including nickel plating, black Imron ${ }^{\circledR}$, polished stainless steel, or black Mylar sheets applied to the model surface. Polished aluminum will yield unacceptable fringes due to an $n_{s} 1.44$, which is too similar to the oil index of refraction to yield sufficient intensity variations. ${ }^{26}$ During sample testing, aluminum 
fringes were hardly visible. Black Mylar sheets offer the most cost effective solution for oil flow testing with an $n_{s}$ of 1.67. However, at higher speeds and long run times, Mylar will begin to peal along the edges, ruining data downstream. The continual Mylar reapplication to the model would prove time consuming and impractical due the model height in the tunnel for the AMELIA test. Mylar is also difficult to apply to a 3D surface, usually resulting in small wrinkles in the Mylar, distorting the skin friction measurements.

Black Imron ${ }^{\circledR}$, polished stainless steel, and nickel plating have the durability that Mylar lacks. The nickel plating $n_{s}$ is 1.6 to 2.0 depending on plating technique. Of the three, a nickel plated surface yields higher fringe intensity variations. Nickel plated aluminum was originally thought to have an overall lower cost and ease of manufacturing than polished stainless steel. For these reasons, a nickel plated aluminum model was chosen for the 2D wing section. However, as Patersonlabs further researched applying nickel plating to the model, additional issues arose. Plating and masking numerous parts would be time consuming and more costly than originally anticipated. The plating requires post polishing, removing any manufacturing benefit over stainless steel. Concern was raised due to the possibility of bending the thin edges on the model during the extensive polishing required of either nickel plating or stainless steel. Portions of the model were excessively large for the nickel plating tanks, so some model parts would go unplated. Interest in a coating of true black $\operatorname{Imron} \AA$, a polyurethane enamel, was discussed with Gregory Zilliac. Patersonlabs found that $\operatorname{Imron}{ }^{\circledR}$ would prove just as durable as stainless steel and nickel plating, but require less working time to yield the surface finish quality desired. The fringe intensity variation of Imron was found to be 
quite good, shown in Figure 9a alongside a comparison to nickel plating, Figure 9b, and stainless steel, Figure 9c. The stainless steel fringes were found to be acceptable, but not as good as the Imron®. The nickel plating yielded the overall best fringe quality. Currently, AMELIA will be painted with Imron ${ }^{\circledR}$ for the application of FISF.

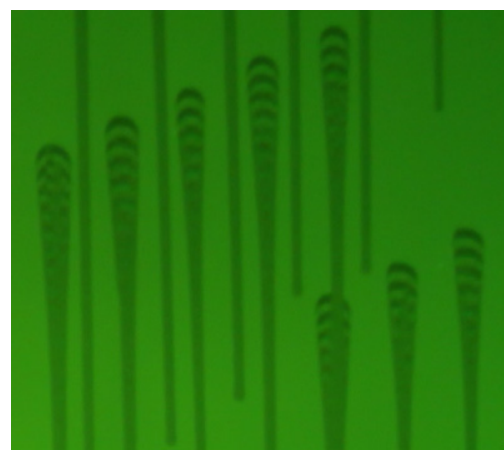

a)

b)

c)

Figure 9. A comparison of fringes on a) black Imron ${ }^{\circledR}$ sample, b) nickel plated wing, and c) stainless steel sample. Each has been polished to a number 2 finish.

\section{Diffuse light source}

In order to properly view the fringes, a monochromatic light source must be reflected off a diffuse reflector. A common method is to build a reflector which encompasses the portion of interest on the model. A small hole is cut in the reflector allowing a camera to capture the area of interest; a similar setup is shown in Figure 10. Another option is to use the tunnel walls as a reflector by covering them with a white material. In large applications, this requires a great deal of lighting and will still fail to illuminate portions of the wing. Once setup however, imaging can be completed in situ, allowing for two datasets per oil run rather than one. To complete this, an initial image is taken once on condition with sufficient fringe spacing. A second image is taken at some time later. The model position is varied. A third image is acquired once on the second condition. A fourth image is taken some time later. By subtracting the fringe spacing between image two and one and image four and three, the change in fringe spacing can be determined for 
the two test conditions. The change in fringe spacing during the time between images can be utilized to yield skin friction for the two test conditions. ${ }^{22}$

Often the FISF technique is applied to models with high curvature as CFD validation test pieces. ${ }^{\dagger, 42}$ The surface curvature causes additional difficulty in imaging, which is illustrated in Figure 11. Due to the mirror finish and curved model surface, the camera will see reflections from a large area of the wind tunnel. Therefore, if the wind tunnel is being used as the diffuse reflector, large areas of wind tunnel must be white and consistent. A diffuse reflector would uniformly light the model with fewer lights, allowing for accurate fringe spacing identification. The diffuse reflector used on the wing blend is shown in Figure 12.

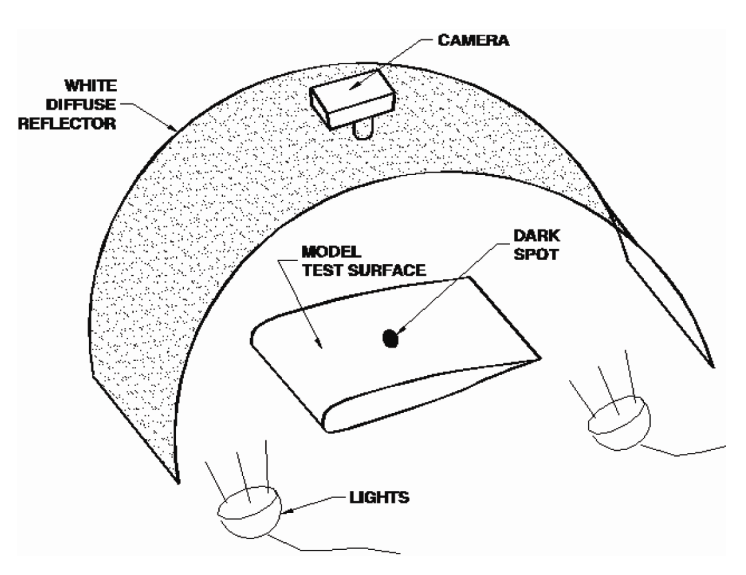

Figure 10. A diffuse reflector being lit from below. The dark spot occurs where the camera lens direction is orthogonal to the model surface. ${ }^{39}$

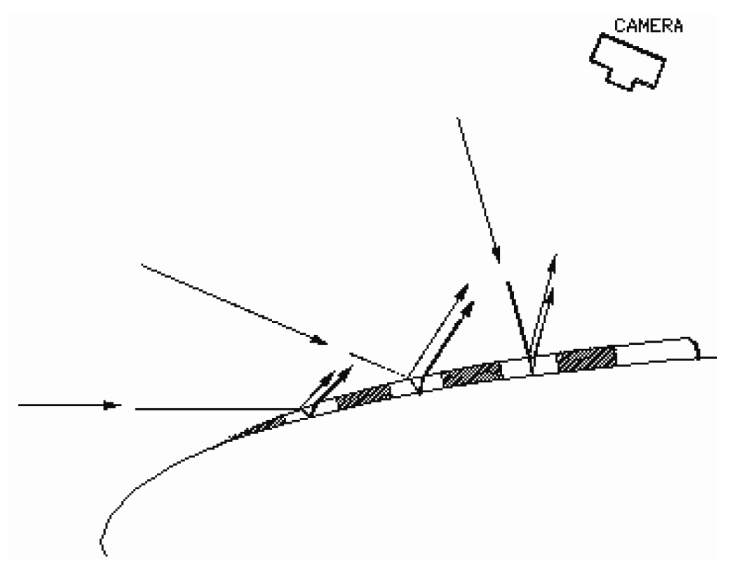

Figure 11. A schematic illustrating that light entering the camera originates from multiple locations, demonstrating the increased lighting difficulty and requirement for model fiducials. ${ }^{39}$

${ }^{\dagger}$ Zilliac, G. G., Private communication, August 2009. 


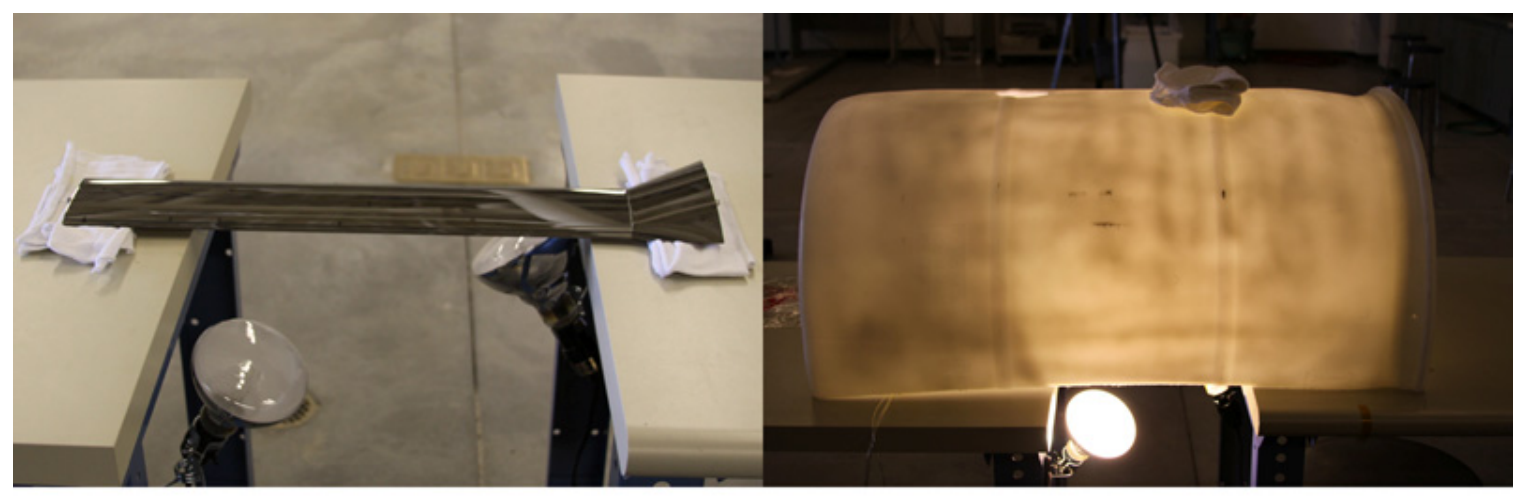

a)

b)

Figure 12. Two photographs of the lighting setup illustrating a) the wing suspended by two tables below and b) a lit white diffuse reflector over the model. The right hole is shown covered with a white cloth.

\section{Fiduciary Markers}

As illustrated by Equation (6), the angle at which the light enters the camera can greatly affect the skin friction measurement, especially at non-orthogonal locations, such as leading edges or the blended wing portion of the model. Zilliac's CXWIN5G software utilizes single camera photogrammetry to determine the light refraction angle between the air-oil interface, $\theta_{r}$. This is made possible by placing fiducial marks over the model surface. The camera captures an image encompassing the entire wing with several fringes over the wing surface. Within that image are multiple fiducial marks with known locations on the model coordinate system. A recommended 12 fiducials yields good results, with a minimum of six required. Zilliac's software completes the photogrammetry by matching the known fiducial marks' locations (both a pixel $x, y$ coordinate system as well as the model $X, Y, Z$ coordinate) to a set of model surface points. This allows the software to calculate the light incident angle at any visible point on the model. The photogrammetry also accurately calculates the fringe spacing on the model, properly converting $x, y$ pixel locations into $X, Y, Z$ locations corresponding to the actual model. ${ }^{38}$ 
Fiducials were not added to the test wing during manufacturing. Initially, fifteen small stickers were utilized as fiducials and applied onto the model somewhat randomly. By placing the wing into a mill, the fiducial's locations were manually determined. Another more reliable method would be to utilize a Faro Arm to determine the fiducials' locations relative to a chosen coordinate system. After initial testing, it was found that the sticker fiducials tripped the laminar flow to turbulent, causing large skin friction gradients on the wing. To remedy this, the stickers were removed and the fiducials were marked with a 0.10 inch circle etched into the model surface with a metal etching pen. The etched fiducials do not trip the flow and are still easily visible. A test was run to illustrate this. Two stars were placed on the model and oil was applied as usual. A normal tunnel run was then completed, with the results in Figure 13.

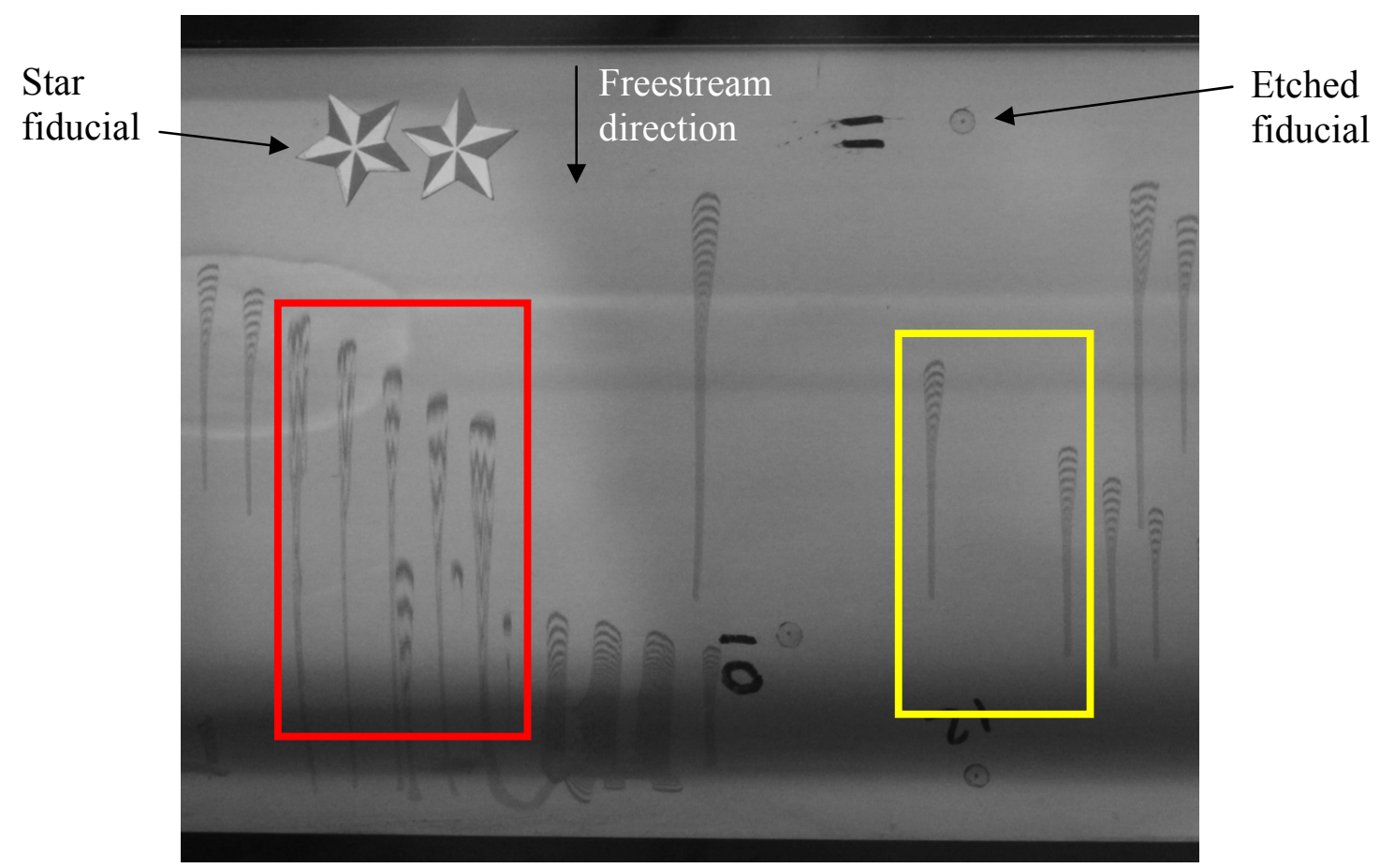

Figure 13. Image of the fiducial test. The fringe spacing behind the stars (boxed in red) is significantly larger than behind etched fiducial (boxed in yellow), indicating the etched fringes do not trip the flow while the star fiducials do. 


\section{Light Source}

The light source needs to be monotonic, allowing an accurate application of interferometry to measure the fringe spacing. Options include fluorescent bulbs, mercury vapor lamps, sodium vapor lamps, or lasers. Sodium has a monotonic wavelength of $589.3 \mathrm{~nm}$. Mercury and fluorescent bulbs both have a strong $546 \mathrm{~nm}$ band which requires filtering to remove any unwanted wavelengths. Self ballasted, 160 watt, mercury vapor lamps from Eye Lighting were chosen to light the model (item number 160WSB/RSP38RF) with manufacturer emission spectra shown in Figure 14. Originally, a $35 \mathrm{~nm}$ full-width half maximum (FWHM) Barco green bandpass filter centered on $546 \mathrm{~nm}$ with greater than $80 \%$ transmittance was chosen. However, upon discussions with Russell Westphal, a more narrow bandpass filter was encouraged to ensure the proper wavelength of light reached the camera. A Semrock FF01-546/6 filter was used, which has a $6 \mathrm{~nm}$ FWHM centered about $546 \mathrm{~nm}$ with greater than $90 \%$ transmittance. The emission lines of the two filters compared to the mercury lamp emission spectra is shown in Figure 15. The Semrock filter has the advantage of allowing the most light in at the desired wavelength. The Barco filter allows a portion of a strong yellow line at $580 \mathrm{~nm}$. This is visible in the images with the Semrock images appearing greener while the Barco images have a yellow tint. However, the extra light from the Barco filter improves the intensity variation of the fringes, shown in Figure 16. The aperture, shutter speed, focal distance, and ISO were held constant between images, with camera held in place with a boom for a true comparison between filters. Utilizing Zilliac's software, the peak to peak fringe intensity variations of the fringes were averaged. Overall, the Barco $35 \mathrm{~nm}$ filter had an intensity variation of 28.5 while the Semrock $6 \mathrm{~nm}$ filter was 23.4, $18 \%$ lower than the Barco filter. 


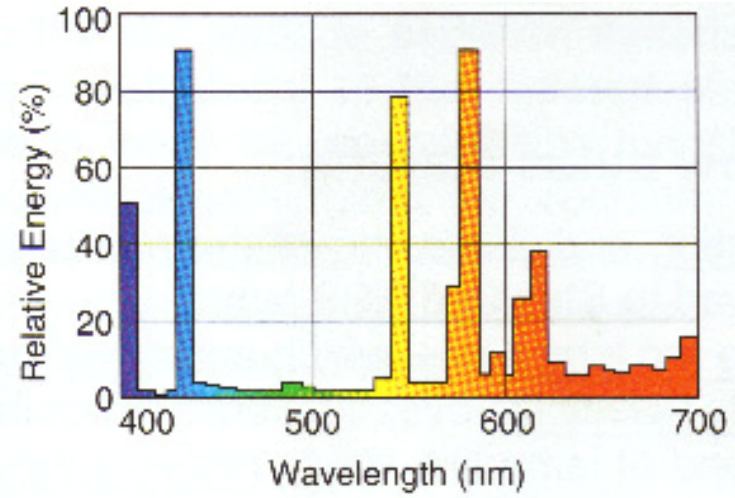

Figure 14. Manufacturer emission spectra of the self ballasted mercury vapor lamps.

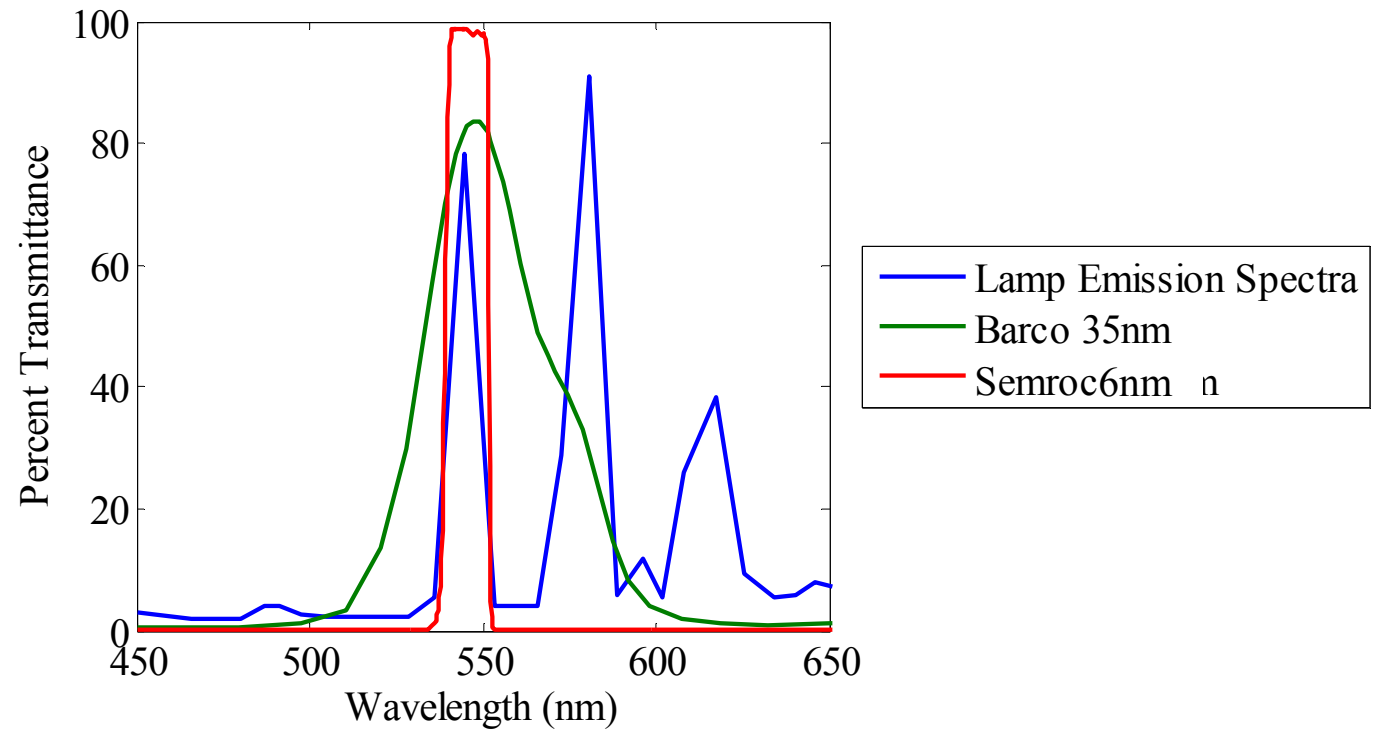

Figure 15. Comparison of the $35 \mathrm{~nm}$ Barco filter and $6 \mathrm{~nm}$ Semrock filter against the mercury vapor lamp emission spectra. Note the mercury lamp spectra is shown with relative scale on the $y$ axis for comparison purposes. 
a)

b)

Figure 16. Comparison of the a) Barco $35 \mathrm{~nm}$ and b) Semrock $6 \mathrm{~nm}$ filter. The aperture, shutter speed, focal distance, and ISO were held constant, with camera held in place with a boom.

\section{Image Acquisition}

Imaging was completed with a Canon 50D Digital SLR. The camera is 15.1 megapixels ( 4,752 by 3,168 pixels), allowing large amounts of data to be obtained from a small number of images. The lens utilized for the data was a Canon EF 28-135mm f/3.5-5.6. To accurately apply the nonlinear curve fitting regression used to measure the fringe spacing, 20 pixels per fringe spacing is recommended. For the frame size, oil viscosity, and run time utilized, the 50D on average yields 25 pixels per fringe for the two foot wing section.

\section{Oil Calibration}

One of the largest sources of uncertainty is oil viscosity due to its strong dependence on temperature. Dow Corning 200 polydimethylsiloxane oil is utilized for skin friction measurements. For the tests completed, nominal $500 \mathrm{cSt}$ and $100 \mathrm{cSt}$ oils were utilized. The oil's viscosity was measured with a calibrated Cannon Fenske Routine Viscometer. The $500 \mathrm{cSt}$ oil was calibrated three times. Throughout the calibration, the temperature remained at $16^{\circ} \mathrm{C}$. 
The oil kinematic viscosity and density are temperature corrected for each run. ${ }^{38}$ The kinematic viscosity is corrected by Equation (7)

$$
v_{o, T}=10^{\wedge}\left[\frac{C_{1}}{T+C_{2}}-\frac{C_{1}}{T_{c a l}+C_{2}}+\log _{10}\left(v_{o, c a l}\right)\right]
$$

where $T$ is a desired temperature in Kelvin, $C_{1}$ is 774.8622 , and $C_{2}$ is 2.6486. The oil's specific gravity can be defined as

$$
\gamma_{o, T}=\frac{\gamma_{o, T=25^{\circ} \mathrm{C}}}{1+\alpha(T-25)}
$$

where $\alpha$ is the coefficient of expansion and $T$ is in degrees Celsius. Properties needed for these calibrations for Dow Corning 200 oil can be found in

Table 1. The oil density is given by

$$
\rho_{o, T}=\gamma_{o, T} \rho_{H_{2} O, T=25^{\circ} \mathrm{C}}
$$

where $\rho$ is the density. This allows for the calculation of $\mu_{o}$ at the run temperature. The uncertainty of the viscometer and oil calibration curve result in a $\pm 1.6 \%$ variability in the 500 cSt oil.

Table 1. Physical properties of Dow Corning 200 oil at $25^{\circ} \mathrm{C}^{43}$

\begin{tabular}{cc|cccccccc}
$v_{\mathrm{o}, \mathrm{nom}}$ & $\mathrm{cSt}$ & 10 & 50 & 100 & 200 & 500 & 1000 & 10,000 & 30,000 \\
\hline$\gamma_{\mathrm{o}}$ & - & 0.934 & 0.96 & 0.964 & 0.967 & 0.969 & 0.970 & - & 0.971 \\
$\mathrm{n}_{\mathrm{o}}$ & - & - & 1.4022 & 1.4030 & 1.4032 & 1.4034 & 1.4035 & 1.4036 & 1.4037 \\
$\alpha_{\mathrm{o}}$ & $\frac{c c}{c c} /{ }^{\circ} \mathrm{C}$ & 0.00108 & 0.00104 & 0.00096 & 0.00096 & 0.00096 & - & 0.00096 & 0.00096
\end{tabular}

\section{Research Model}

A two foot wing section was manufactured by Patersonlabs that is representative of Cal Poly's large scale wind tunnel model for the AMELIA test, shown in Figure 17. A front view of AMELIA is shown in Figure 18 as a comparison to the test article in 
Figure 19. Ideally, a scale model of the AMELIA wing blend would have been made, but based upon material readily available at Patersonlabs, the $2 \mathrm{D}$ wing section was limited to a six inch chord and three inch overall thickness. For this reason, a long 2D section was machined, with a small 3D section on the inboard. The airfoil on the left side of Figure 19 is a NLR-7301. This airfoil blends down to a modified SC2-0414 2D section with a chord of 3.55 inches. The lower surface of the SC2-0414 was modified to simplify the blended portion. This was not critical as the model is intended as a research tool for uncertainty and test methodology development. The SC2-0414 is set to a positive $1.08^{\circ}$ angle of attack relative to the NLR-7301. All angle of attack measurements were with reference to the NLR-7301.

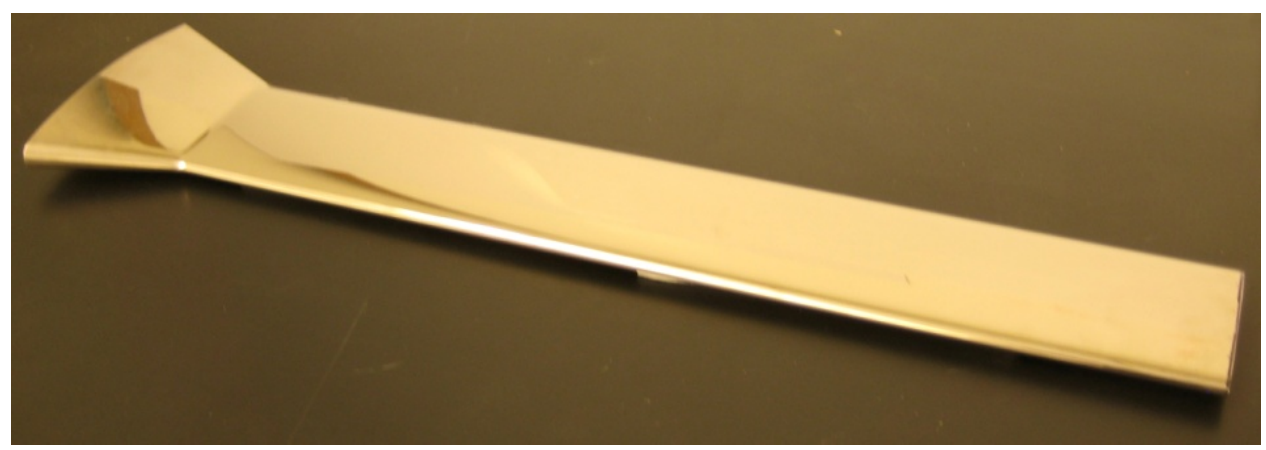

Figure 17. A photograph of the nickel plated test article, showing wing blend at far left.

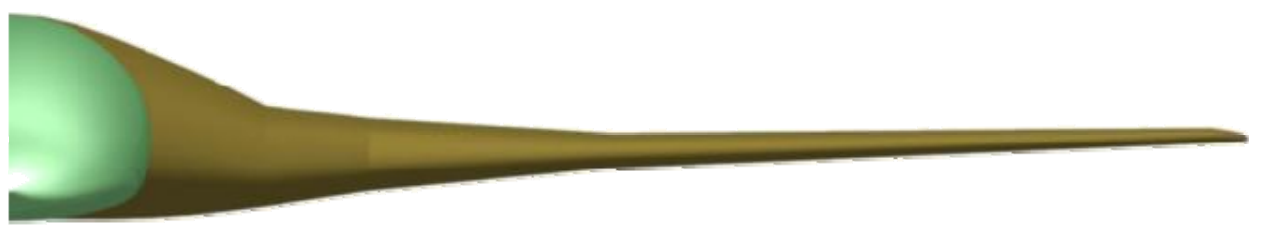

Figure 18. Front view of AMELIA cut on the symmetry plane, illustrating the wing blend to fuselage (shaded green). 


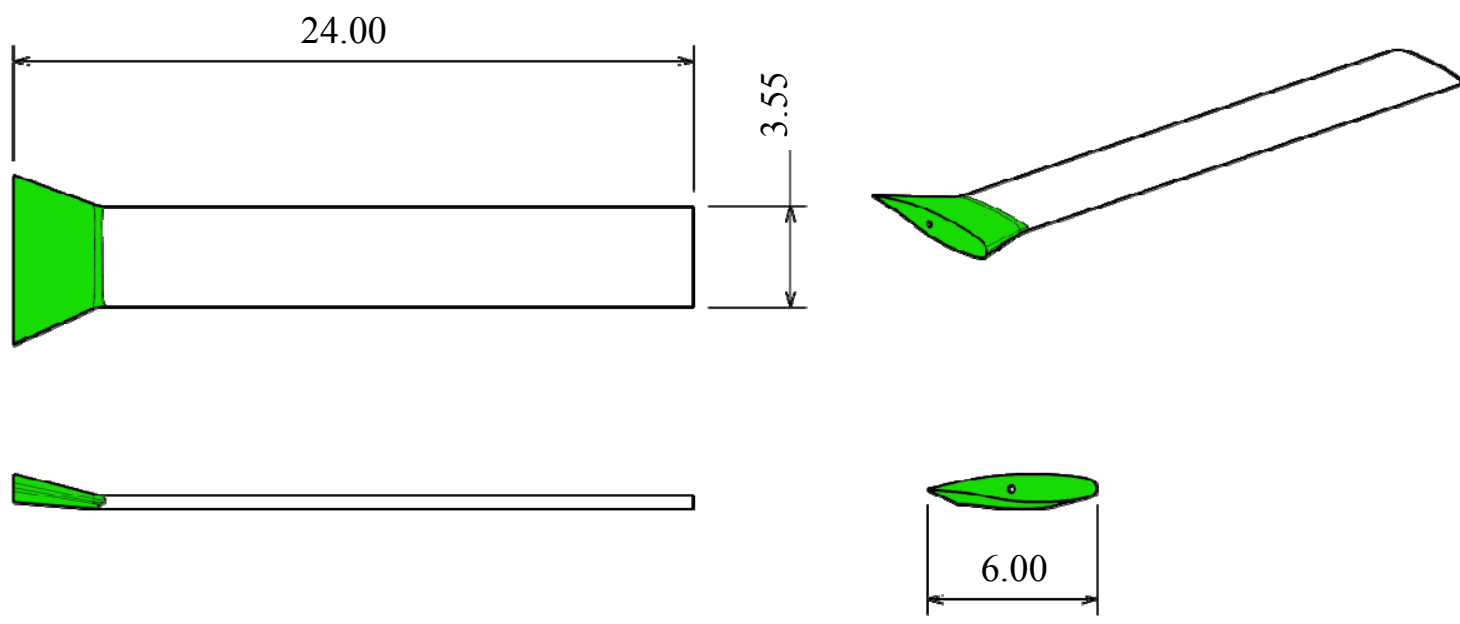

Figure 19. Three view of blended wing where the 3D wing blend section is shaded green and the $2 \mathrm{D}$ section is white (all dimensions are in inches).

\section{Test Procedure}

Testing was completed in the Cal Poly Mechanical Engineering two foot by two foot wind tunnel, shown with model in Figure 20. The tunnel was manufactured by Engineering Laboratory Design, Inc. It is an open circuit Eiffel style tunnel capable of velocities up to 160fps. The test section is four feet long. The maximum Reynolds number for the $2 \mathrm{D}$ test article chord is 285,000 , obtained with a freestream velocity of 163 fps. All tests were run at this Reynolds number. The model is first coated in oil droplets applied with a plastic comb. Care must be taken to ensure the drops are not too large or in line with one another. Large oil drops may flow into one another in the spanwise direction before the wing is tested, creating an oil line. If drops are placed in line with one another, the oil from the upstream drop will flow into the downstream drop, possibly ruining the downstream fringes. Once the drops are applied, the wing is placed in the tunnel at roughly a $30^{\circ}$ angle of attack. As the tunnel starts, the oil slightly shears due to gravity and the recirculation region formed behind the airfoil; the thinning during this time is considered negligible. The airfoil is then set to the desired angle of attack and run for approximately 9 to 16 minutes depending on oil viscosity, laminar or turbulent 
flow conditions, and tunnel speed. The angle of attack is set with a digital level relative to the NLR-7301 airfoil with $\pm 0.1^{\circ}$ accuracy. If the fringes are not allowed to fully develop, the camera cannot capture sufficient resolution to view the fringes properly. Conversely, if the tunnel is run too long, the fringes will become irregular and difficult to accurately image. In situations where fringe spacing cannot be visually observed, Zilliac ${ }^{\ddagger}$ has developed a script to determine the ideal oil viscosity depending on run time, freestream dynamic pressure, and whether the flow is laminar or turbulent. Once the fringes are developed to an appropriate spacing, the airfoil is set back to $30^{\circ}$ angle of attack while the tunnel is shut down. The wing is removed from the tunnel and imaged.

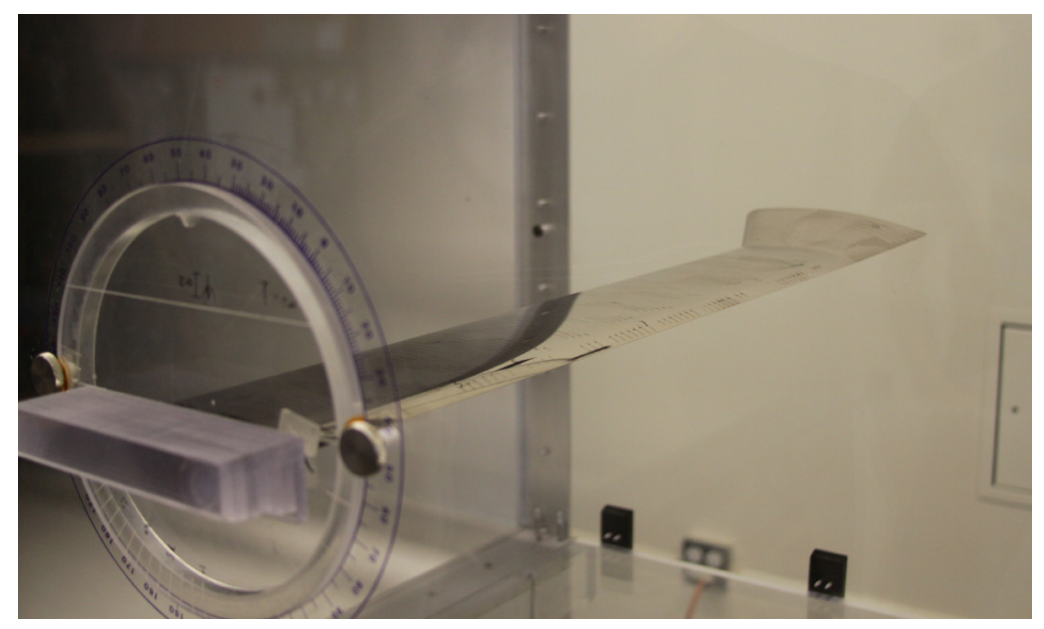

Figure 20. Image of the 2 foot wing section in $2 \times 2$ foot wind tunnel with access plate visible on far left.

This test procedure was utilized to mimic the procedure planned for AMELIA. Due to the long transients in the NFAC (approximately 10 minutes for startup and shutdown combined), extremely long run times were going to be required to sufficiently lower the transient uncertainty. Instead, AMELIA will similarly be pitched to where the flow is separated. Once the desired freestream dynamic pressure is reached, the model will be set to the desired location with the slot flow and turbine propulsion simulators turned on. \$ Zilliac, G. G., Private communication, May 2010. 
Upon test completion, the model will be reset to a separated angle of attack during the tunnel shutdown. Any gravity or recirculation effects are considered negligible due to the high viscosity of the oil and the comparatively low recirculation velocities.

\section{Experimental Uncertainty}

Zilliac extensively outlines uncertainty due to numerous factors, summarized and compared against a completed run in Table $2 .{ }^{32}$ These sources of error are all a certain component of Equation (6). They can be easily combined to estimate the total skin friction coefficient uncertainty by applying the constant-odds uncertainty analysis technique from Kline and McClintock. ${ }^{44}$ The oil initial condition was estimated to be the lowest uncertainty due to a run time exceeding 900 seconds. The observed datasets focused upon the 2D portions of span on the model, thus oil streamline curvature errors can be assumed negligible. The oil viscosity uncertainty is directly dependent upon the freestream temperature and was determined to be $\pm 1.6 \%$ of $v_{o}$. Due to the low velocity flow, the oil temperature was assumed to have greater accuracy than the uncertainty range given by Zilliac. However, since the oil was not calibrated at the test run temperature, a calibration curve was utilized to calculate the corrected viscosity, adding additional error. The freestream dynamic pressure varied $\pm 2.14 \%$ of $q_{\infty}$, which is greater than Zilliac's range due to the quality of the wind tunnel in use. The shear and pressure gradients were assumed to be zero. The regression and imaging errors were considered to be the accuracy of the nonlinear regression curve fitting the fringe intensity data. Utilizing output data from Zilliac's software, the regression error was calculated for ten points and averaged for the entire run. However, the regression error does not directly correlate to error in the measurement. The curve fit may be poor, but the fringe spacing 
measurement can still be extremely accurate. CXWIN5G does not make adjustments for lens distortion; therefore, changes must be made beforehand. Lens distortion errors were found to be negligible. Lastly, due to the procedure described above, the tunnel transient errors were removed. The following uncertainty analysis yields an error bound of $-3.7 \%$ to $+3.6 \%$ of $c_{f}$, ignoring possible filter variations. The direction of the skin friction coefficient vectors is assumed to have $\pm 1.0^{\circ}$ accuracy, as listed by Zilliac. ${ }^{32}$ This calculation covers a broad range of uncertainties within the technique; however, additional uncertainties are developed below.

Table 2. Summary of Zilliac's FISF uncertainty sources. ${ }^{32}$

\begin{tabular}{lcc} 
Error source & Uncertainty range & Wing blend uncertainty \\
\hline Oil initial condition & $-0.03 \%$ to $-0.2 \%$ of $c_{f}$ & $-0.03 \%$ of $c_{f}$ \\
Oil streamline curvature errors & $0 \%$ to $5 \%$ of $c_{f}$ & $0 \%$ of $c_{f}$ \\
Oil viscosity & $\pm 0.2 \%$ to $\pm 5 \%$ of $v_{o}$ & $\pm 1.6 \%$ of $v_{o}$ \\
Temperature & $\pm 0.05 \%$ to $\pm 1 \%$ of $T_{o}$ & $\pm 0.012 \%$ of $T_{o}$ \\
Light source wavelength & $\quad$ very small & filter dependant \\
Oil index of refraction & $\pm 0.015 \%$ of $n_{o}$ & $\pm 0.015 \%$ of $n_{o}$ \\
Freestream dynamic pressure & $\pm 0.25 \%$ to $\pm 1.0 \%$ of $q_{\infty}$ & $\pm 2.14 \%$ of $q_{\infty}$ \\
$d P / d s$ & $\pm 0 \%$ to $\pm 0.14 \%$ of $c_{f}$ & $\pm 0 \%$ of $c_{f}$ \\
$d \tau_{w} / d s$ & $\pm 0 \%$ to $\pm 20 \%$ of $c_{f}$ & $\pm 0 \%$ of $c_{f}$ \\
Regression and imaging errors & $\pm 0.5 \%$ to $\pm 5 \%$ of $\Delta s$ & $\pm 2.5 \%$ of $\Delta s$ \\
Lens distortion errors & $\pm 0 \%$ to $\pm 1.4 \%$ of $\Delta s$ & $\pm 0 \%$ of $\Delta s$ \\
Startup and shutdown $c_{f}$ errors & $0 \%$ to $?$ & $0 \%$ of $c_{f}$
\end{tabular}




\section{Human Repeatability}

In CXWIN5G, the user manually creates lines over oil drops, representing the skin friction direction. The line over the image is the location where data (intensity and pixel location) are interpolated. The data are plotted in the form of intensity versus pixel location, as illustrated in Figure 21. A nonlinear regression is applied to the data. The two blue vertical lines (at pixel locations 18 and 45 in Figure 21) represent the minima of the first two dark fringes. The distance between the two blue lines is the fringe spacing.

Since the oil droplet method is being implemented, care must be taken to place the data line on the oil droplet centerline. Off center placement will yield inaccurate fringe spacing measurements due to 3D spreading effects. Additionally, the oil droplet directional accuracy is dependent upon the user's line placement.
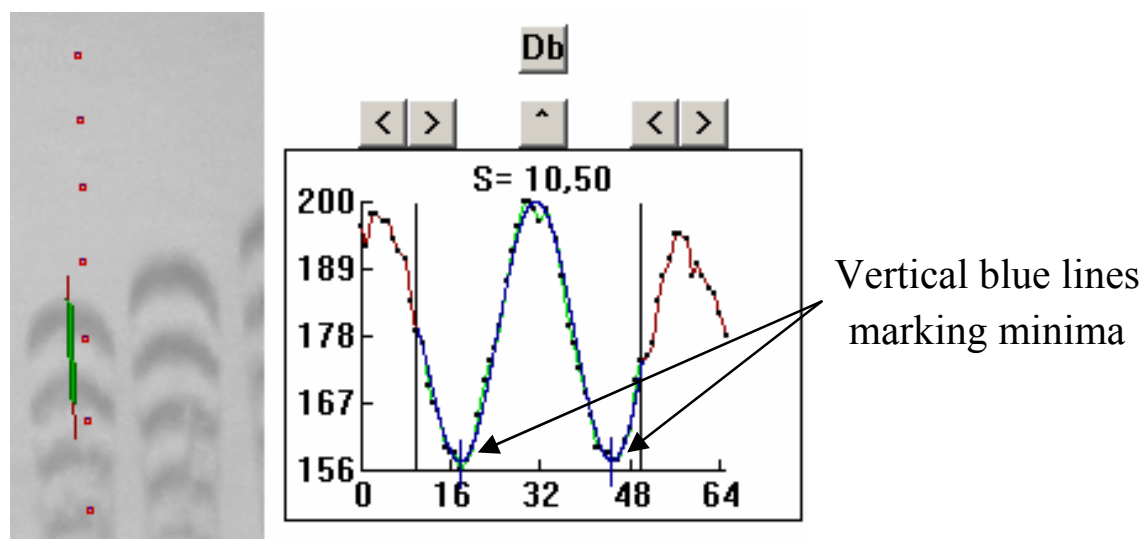

Figure 21. Screenshot of data acquisition software, with green line on the left directly corresponding to fringe intensity versus pixel location graph on the right.

To characterize the uncertainties, ten oil fringe spacings were each measured fifty times over the course of a month. All other variables were held constant, yielding only the human repeatability uncertainty. The findings are reported in Table 3. The fringe spacing varies from $\pm 1.00 \%$ to $\pm 2.34 \%$ while the direction varies from $\pm 1.36^{\circ}$ to $\pm 2.03^{\circ}$. Data point 6 is an outlier with a $\pm 19.36^{\circ} 95 \%$ confidence interval. Human directional accuracy is much higher as indicated from the remaining nine data points. Hence, there 
must be a photogrammetry error occurring which yields the large angle variation. If data points seem unreasonable, the CXWIN5G software easily allows removal or updates of the data.

According to Zilliac, $\mathrm{a} \pm 1^{\circ}$ accuracy is achievable with care, which is slightly lower than the findings presented here. By allowing the fringes to develop longer and increase fringe spacing, the accuracy of the centerline placement would improve. However, as the oil continues to thin, the fringe quality decreases and streamwise streaks begin to appear. The streaks make fringe spacing measurements difficult and inaccurate, illustrated in Figure 22.

Table 3. Summary of uncertainty due to human repeatability. Point 6 is a directional outlier.

\begin{tabular}{ccc} 
Point & $d \Delta s / \Delta s(\%)$ & $\pm 2 \sigma\left(^{\circ}\right)$ \\
\hline 1 & 1.68 & 1.60 \\
2 & 2.03 & 2.03 \\
3 & 1.00 & 1.74 \\
4 & 2.07 & 1.59 \\
5 & 2.09 & 1.36 \\
6 & 2.34 & 19.36 \\
7 & 1.87 & 1.90 \\
8 & 1.82 & 1.55 \\
9 & 1.90 & 1.83 \\
10 & 1.25 & 1.70
\end{tabular}


Figure 22. Image illustrating $500 \mathrm{cSt}$ oil fringes on the left compared against large fringe spacing of 100 cSt oil on right.

Efforts by Naughton ${ }^{45}$ and Zilliac $^{38}$ have been made to automate the fringe measurement technique. Naughton developed an automated fringe detection method utilizing a windowed Fourier transform to locate the fringes and a correlation function to measure the fringe spacing. This software was intended to be applied to Zilliac's CXWIN5G, but to date has not been fully utilized. Zilliac's current version requires the user to place a point near the center of a fringe. The system automatically locates the fringe locations and chooses the most appropriate line representing the fringe spacing and direction. The user is allowed to vary the points utilized in the nonlinear regression and determines whether the automation yields appropriate results. The future implementation of these methods will improve the variation in human measurements.

\section{Photogrammetry}

Photogrammetry allows an $x, y$ pixel location in an image to be correlated to an $X, Y$, $Z$ model location. The accuracy of the photogrammetry is dependent upon the model's tolerances and the placement and number of fiducials. CXWIN5G outputs a root mean square of the residual in pixels. However, a more direct comparison of the photogrammetry error was desired, requiring a different uncertainty methodology. A sinusoidal fringe pattern was created in MATLAB and printed onto paper. These known 
fringes were taped onto the wing blend in various locations. An image of the wing was acquired and post processed utilizing the photogrammetry in CXWIN5G, shown in Figure 23. Since the fringes have an ideal intensity distribution, the nonlinear regression uncertainty is assumed to be negligible. Human repeatability uncertainty was reduced by utilizing fringes with square edges, aiding the user to place the data line as accurately. Also, since the fringe is $2 \mathrm{D}$, the data line horizontal placement has no effect on the fringe spacing.

Of 177 data points (multiple points taken per fringe), the average fringe spacing was determined to be 0.2032 inches, while the known fringe spacing was 0.2000 inches, yielding a positive $1.60 \% \Delta s$ skew, within the uncertainty from human repeatability. The fringe measurement had a $95 \%$ confidence interval of $5.37 \%$ of $\Delta s$, which is larger than the human repeatability error. If the data line is placed non-orthogonal to the oil fringe location, the fringe spacing measurement will be greater than the actual length. This yields a positive skew in the fringe spacing measurement. To determine if the positive skew is due to inaccuracies in the data line placement, the inverse cosine of 0.2032 and 0.2000 inches was taken, resulting in $10.18^{\circ}$. This is much greater than the maximum of $2.03^{\circ}$ due to human repeatability. This illustrates that the majority of positive skew in fringe spacing was not due to non-orthogonal data line placement. The positive skew due to photogrammetry has been observed in data output as well, with leading and trailing edge data locations being skewed forward and backward, respectively. Utilizing a 95\% confidence interval, the fringe spacing has a $-6.9 \%$ to $+3.8 \%$ uncertainty range due to the skew. 
A second way to characterize the photogrammetry uncertainty is to create a contour plot of the uncertainty over the wing surface, as shown in Figure 24. This method is advantageous in that the uncertainty of various locations is shown, not simply the global average. By investigating Figure 24, it is clear that the high uncertainty locations are not outliers, but areas of photogrammetric uncertainty. Regions of high uncertainty occur due to poor fiducial placement. Since there is high curvature at the leading and trailing edges, the fiducial inaccuracies will have the largest effect at these locations.

The photogrammetry uncertainty analysis completed holds true only for images calibrated on the 2D non-blended portion of the model. A similar methodology could be utilized for other models. It is noted that Naughton and Brown ${ }^{36}$ assumed that the photogrammetry error was negligible for accurately resolved models. It is good practice however to determine the accuracy of any photogrammetry calibration before data acquisition occurs.

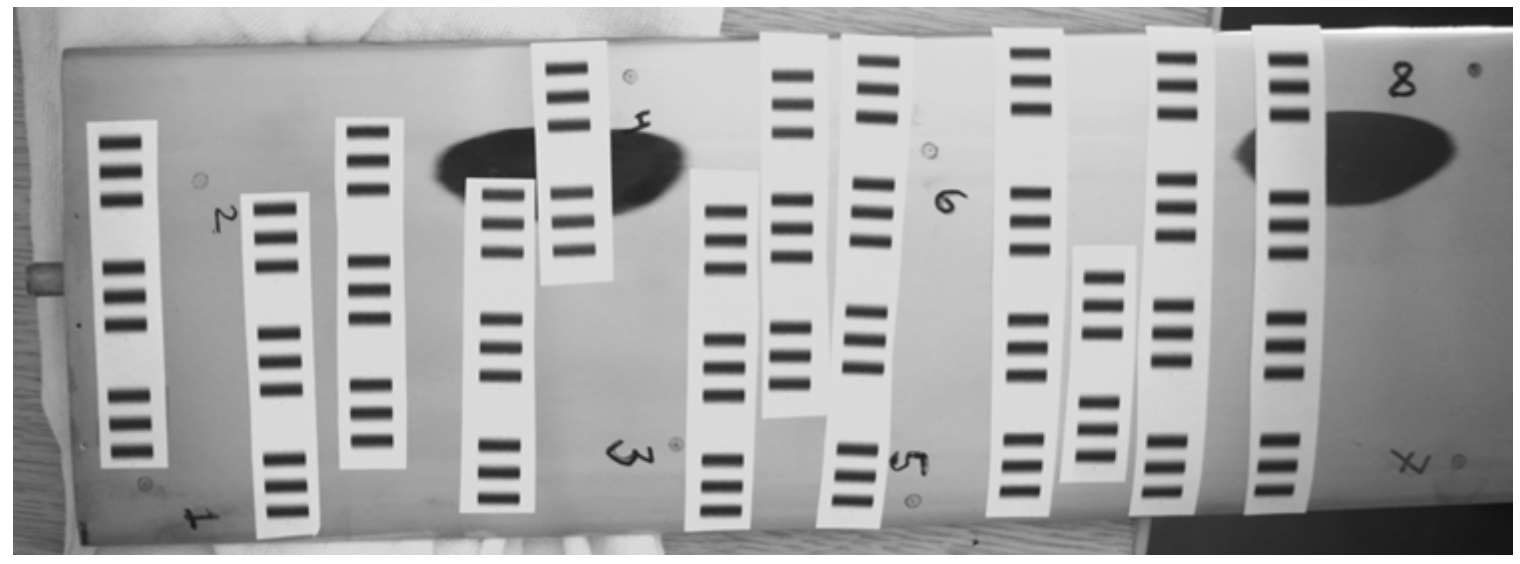

Figure 23. Image of 2D portion of wing blend with known fringes applied to model surface. 


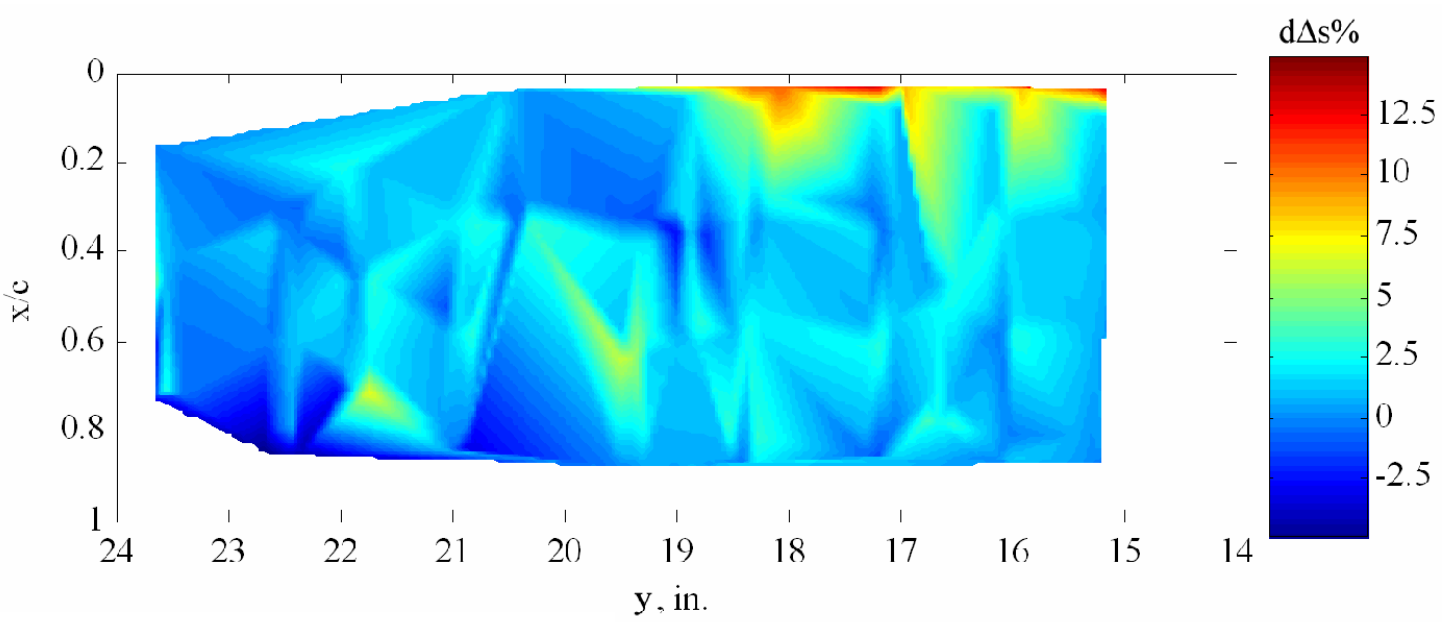

Figure 24. Contour plot of percent fringe spacing error relative to the location on the wing. The leading edge is particularly poor, while the majority of points are more accurate.

\section{Experimental Repeatability}

Since AMELIA will not have the opportunity to complete multiple oil data runs, the experimental repeatability was investigated. An issue arose when comparing two datasets from different days. Since data is only acquired at oil fringe locations, interpolation is utilized to compare the data in a meaningful manner at a given span location. The data does not always compare well if an area lacks oil drops. However, as shown in Figure 25, the data still agrees within the experimental uncertainty with eight days between tests. From 0.7 to $0.9 x / c$, the skin friction drops much lower in Run 2 compared to Run 1 . One possible explanation of the variation is that the flow in Run 1 could have begun to transition at $70 \%$ chord, with a Reynolds number of 200,000. A span location between 20 to 22 inches shows fully turbulent flow at the trailing edge depending on the run, despite utilizing a clean wing. It is likely that transition is occurring at various locations on various wind tunnel runs.

Overall, the data shows good correlation as a test method. However, at $40 \%$ chord, there is an $11.8 \%$ difference between skin friction coefficients. This is most likely due to 
an interpolation error from a lack of data at the location of interest. A trip strip would improve the data comparison by controlling the location of transition.

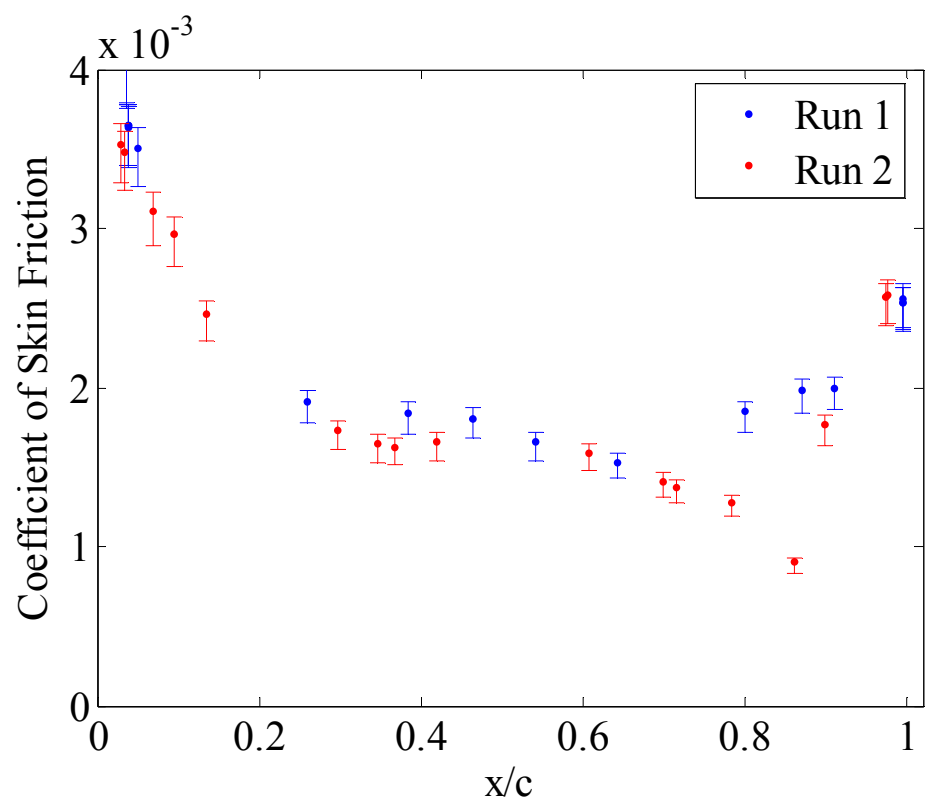

Figure 25. Experimental repeatability of two runs performed eight days apart at a span of 16 inches. A greater variation in data occurred due to interpolating data points at different locations.

\section{Camera Focus Variation}

The optical requirements for the model result in a near mirror surface finish quality. When attempting to image oil fringes, the camera autofocus will focus on what is seen in the reflection, not the actual fringe. Often the camera will not capture an image due to its inability to autofocus. Therefore, manual focus is utilized while imaging fringes. Similarly for the camera autofocus, it is difficult to discern whether the image is in focus or not, since much of the image detail is small. Due to the costly nature of testing in the NFAC, the imaging process must be streamlined to minimize downtime. Therefore, it is important to investigate the degree to which an image's focus varies the measurement accuracy. 
First, the camera's range of focus at differing distances was determined. If a range of acceptable focus distances is known, a more accurate estimate of the correct focus can be made while imaging fringes. This proves valuable if unable to look through the viewfinder while imaging. Focus rings on lenses are ridged to make manual rotation of the ring easier. The ridges were utilized to improve the resolution of the focus variations. However, the focus distance for each ridge is unknown. To quantify the focus variations, the following test was completed. A $28-135 \mathrm{~mm}$ lens was used, with the focal length set to $28 \mathrm{~mm}$. The macro setting on the lens is quoted to focus at a minimum of 19.7 inches. To remove human judgment from the focus determination, the camera's focus indicator was utilized. The nearest image capable of focusing on ridge 1 was 16.4 inches, 3.3 inches closer than the manufacturer specifications. Figure 26 illustrates that as the object distance is moved away from the camera, the acceptable range of focus increases. By fitting a linear approximation to the data, the focus variation per ridge was determined. Roughly, for ridges 1 to 10 , the focus distance changes by one inch per ridge.

To test how focus variation affects fringe quality, multiple images were taken with a varying focus distance, illustrated in Figure 27. The camera was placed on a boom, with a set shutter speed (1/32 s), aperture $(f / 6.4)$, and focal length $(30 \mathrm{~mm})$. The focus distance was set to the shortest length (ridge 1), approximately 17 inches from the CCD plane to the model surface. The focus distance was increased by one ridge increments nine times. The image was most in focus at focus distances of 19 to 20 inches. As the image goes out of focus, the fringe variation blurs. Only the highest intensity variation and longest spaced fringes were acquirable. Figure 28 summarizes the data acquired at a span location of 14 inches. The error bars represent error only due to human repeatability. A 
"ridge" represents approximately one inch of focus distance variation. Ridges 3-6 represent excellent correlation with one another. Ridges 1 and 2 lack in data points, but contain data which matches well with the focused data in general. Ridges 7 and 8 however differ greatly in accuracy; numerous points fail to match within the human repeatability uncertainty. An image with ridge 9 was attempted but the quality was far too low, yielding only data from the high quality leading edge fringes.

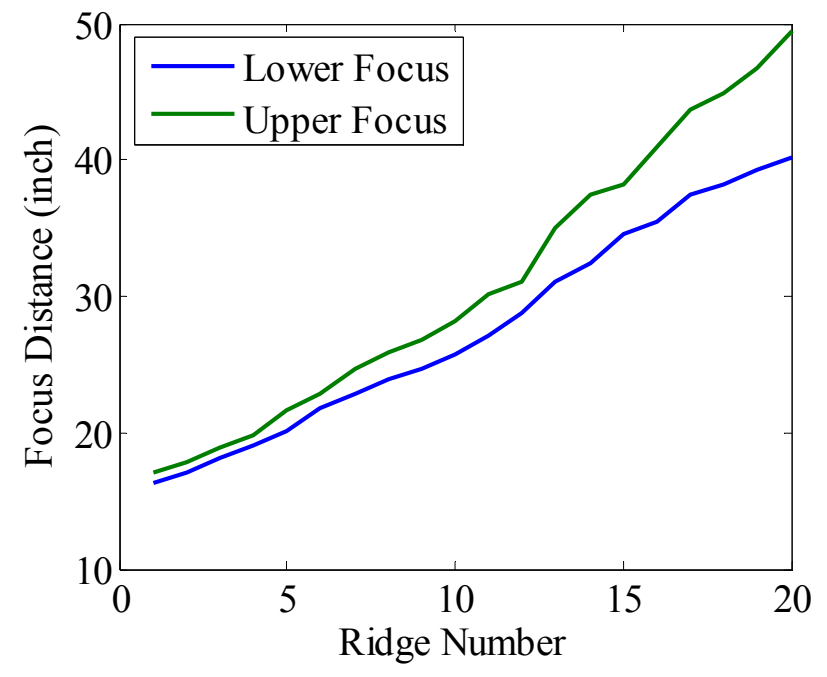

Figure 26. High and low limits of the focus distance for various lens focus positions.

It was found that \pm 2 inches in focus distance yielded data within average human repeatability errors of $\pm 1.8 \%$. At larger focal lengths, the range of acceptable focus distance increases. Beyond this, the data varied greatly. If a few fringes have marginal focus, it is acceptable to acquire data from them. If the vast majority of fringes are not clear, the image should not be utilized for FISF. Figure 27 clearly shows how poor focus effects fringe quality. 
Figure 27. Image focus variation from ridge 1 to 8 , with three oil drops shown and one fiducial.

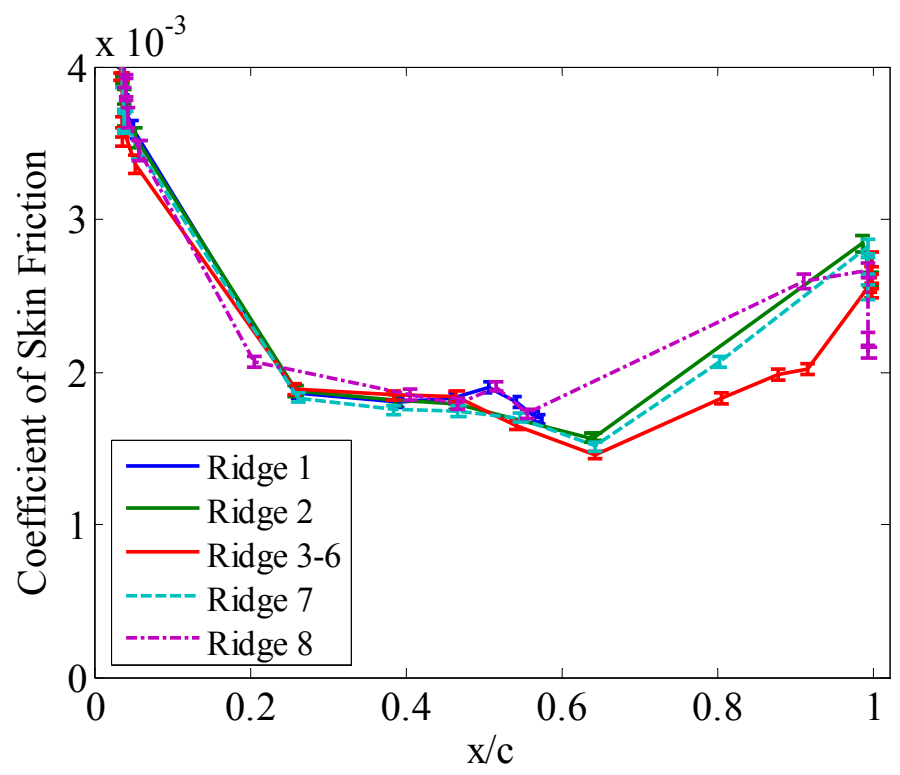

Figure 28. Coefficient of skin friction versus $x / c$ location at span location 14 inches. Ridge numbers represent focus distances, with 4 having the best focus and 1 and 8 the worst.

\section{Filter Bandpass Variation}

In order to calculate the skin friction, the wavelength of light must be known. Quasimonochromatic light sources are utilized to yield strong wavelength bands. A bandpass filter is placed in front of the camera lens to allow only one wavelength of light into the camera. A $6 \mathrm{~nm}$ and $35 \mathrm{~nm}$ FWHM bandpass filter were compared, shown in Figure 29. The $6 \mathrm{~nm}$ is an extremely narrow, high quality filter, allowing more than $90 \%$ transmittance. However, there is an appreciable blue shift from non-orthogonal light entering the filter. Since the light source is quasi monochromatic without a blue band, a dark ring appears around the image limiting the useful data. The $35 \mathrm{~nm}$ filter is lower quality and allows $70 \%$ transmittance. Due to the broader FWHM, a full frame can be 
captured. Note that the shutter speed, aperture, and focal length were held constant for these two images, while the camera position was maintained with a boom. Figure 30 illustrates that the data from the two filters lies within the uncertainty bounds for human repeatability. Other span locations were investigated; no bias was seen in the data. The $6 \mathrm{~nm}$ filter did not consistently yield higher or lower data compared to $35 \mathrm{~nm}$ filter.
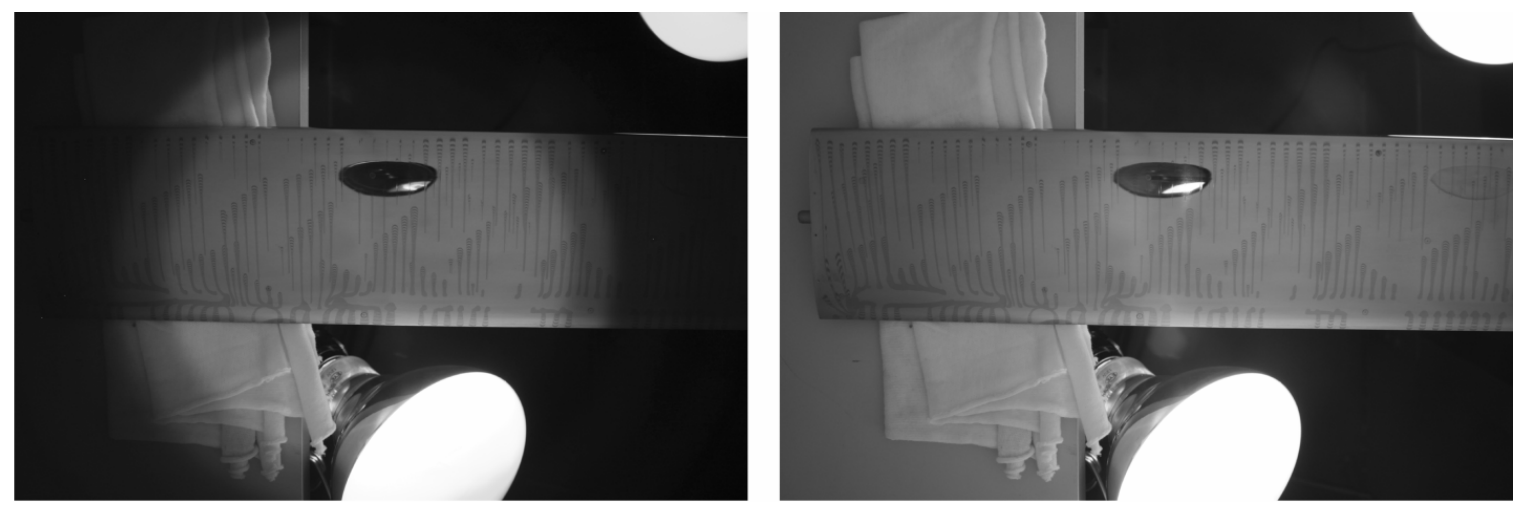

Figure 29. Comparison of the a) $6 \mathrm{~nm}$ Semrock filter illustrating dark ring to the b) $35 \mathrm{~nm}$ Barco filter.

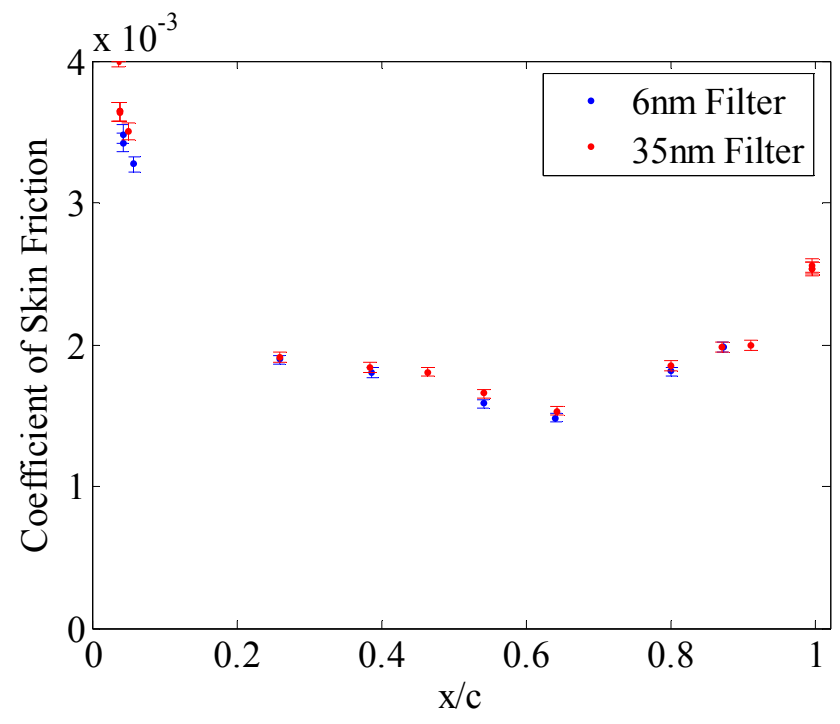

Figure 30. Skin friction coefficient versus $x / c$ at a span location of 14 inches for 2 bandpass filters.

Particular care should be taken while attempting to image the edges of data from the $6 \mathrm{~nm}$ filter. Since a blue shift occurs to $530 \mathrm{~nm}$ in the filtering (attenuating the $546 \mathrm{~nm}$ 
band), only the shifted light is entering the lens at these locations. Since the wavelength is different, a 3\% inaccuracy will occur in the dark ring areas.

\section{High Dynamic Range and Additional Post Processing}

Although generally not encouraged, the possibility in improving the oil data exists through the use of High Dynamic Range (HDR) or additional post processing. To ensure the possible data variation was acceptable, HDR was applied to both the $6 \mathrm{~nm}$ and $35 \mathrm{~nm}$ filter images. The $6 \mathrm{~nm}$ filter would benefit greatly from post processing in that the outboard fringes could possibly be acquired. Again, due to the blue shift, a correction would need to be made for the variation in wavelength, up to a $3 \%$ variation in the data.

Each HDR photo had three images at varying exposures taken. A boom held the camera over the wing to ensure minimal position variation. Adobe Photoshop ${ }^{46}$ was utilized to post process the HDR images. Photoshop imports the photos, aligns them with one another to remove any small inconsistencies in framing, and creates an image with a higher dynamic range, thereby increasing the range of luminance in the image. The results are shown for the two filters in Figure 31 and Figure 32.

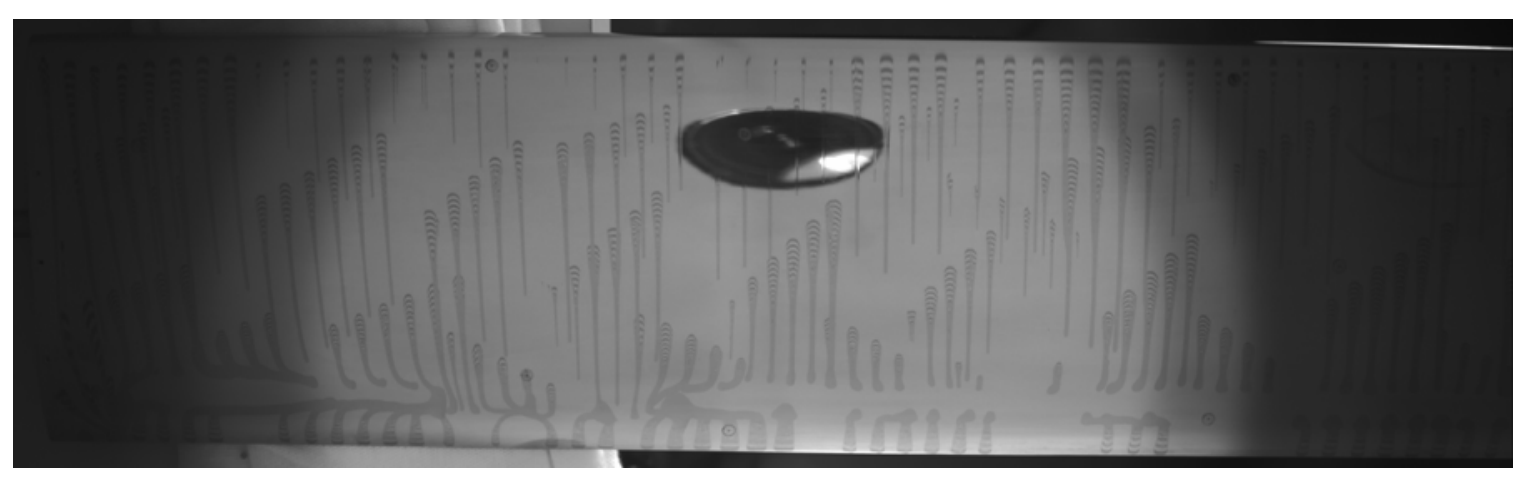

Figure 31. HDR image taken with Semrock $6 \mathrm{~nm}$ bandpass filter with camera reflection clearly visible. 


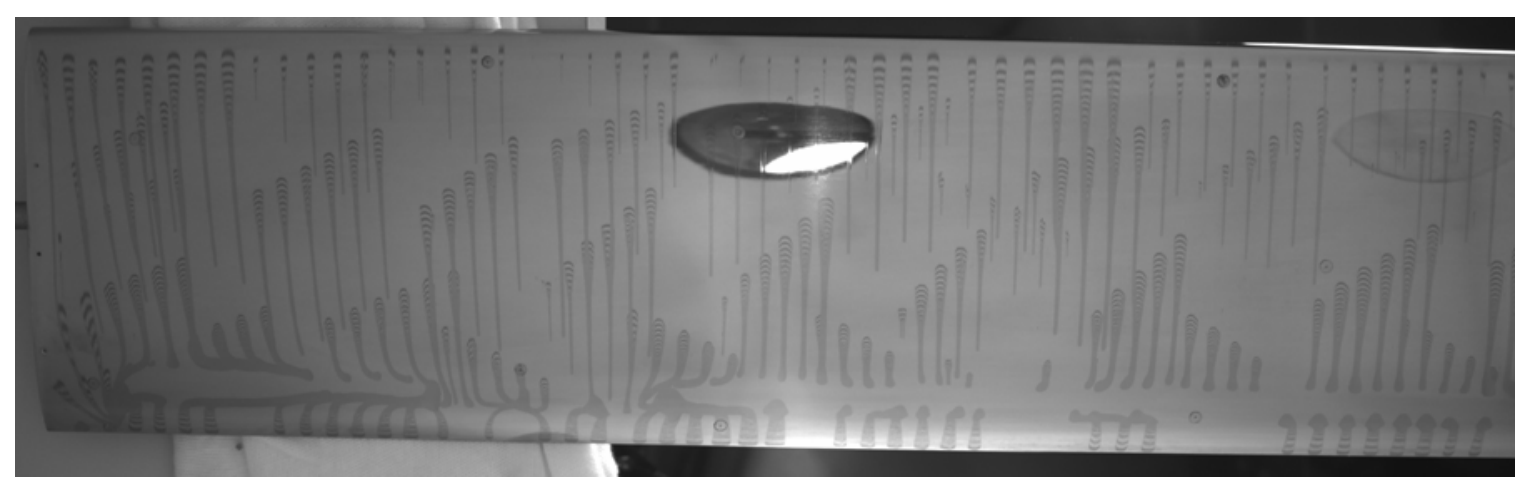

Figure 32. HDR image taken with Barco $35 \mathrm{~nm}$ bandpass filter. Note the high noise region near the center of the image.

It was found that limited improvements could be made with HDR. If the edge fringes were better illuminated, the center image fringes became noisy and beyond use. This is illustrated in Figure 33 with the green, $35 \mathrm{~nm}$ HDR data points clearly offset in the 0.1 to $0.4 x / c$ location (center of the image). However, the data at the leading and trailing edges are closer to the original. HDR images are not recommended.

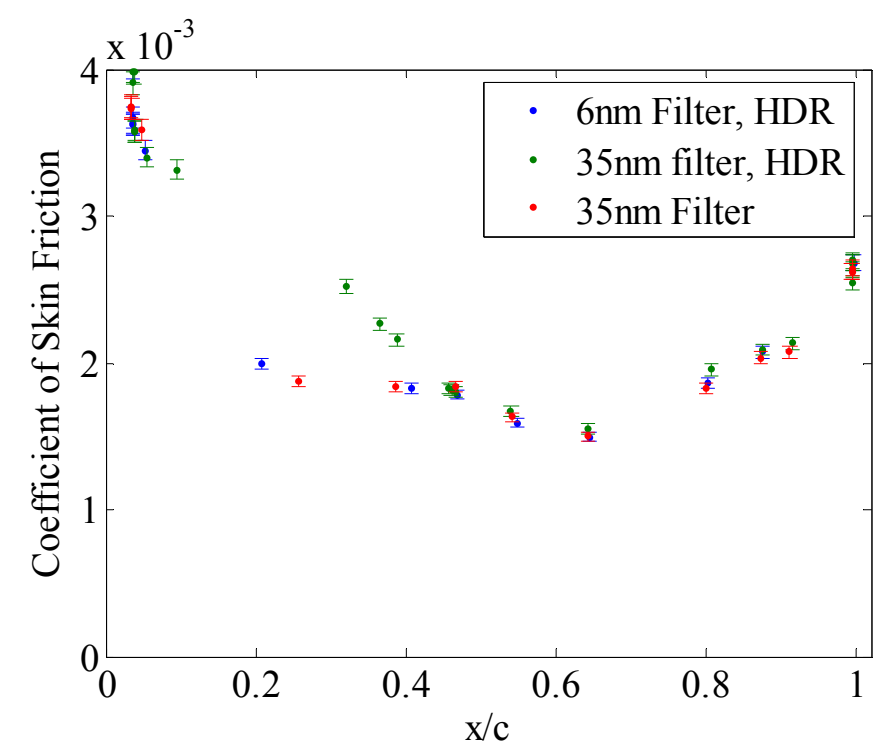

Figure 33. Skin friction coefficient data at a span location of 14 inches. The $35 \mathbf{~ n m}$ filter without HDR adjust illustrates a consistent variation against the HDR data.

\section{Varying Oil Viscosity}

Typically the FISF technique is conducted with oil of a single viscosity. However, oils of differing viscosity can be used simultaneously for instances with a large variation 
in shear stress as long as the viscosity of individual droplets or regions is documented. This is useful in tests where large variations in velocity over the model surface occur. In preparation for AMELIA testing, an investigation of the difficulties and requirements of testing with oils of differing viscosity was conducted.

Early testing of the two foot blended wing section indicated separated flow at angles of attack greater than $5^{\circ}$ for a freestream dynamic pressure of 30.7 psf. This condition provided an opportunity to utilize two oil viscosities simultaneously. As an experimental control, the test article was divided into equally spaced regions along the span, where droplets of $100 \mathrm{cSt}$ and $500 \mathrm{cSt}$ oils were applied at certain span locations. The test was conducted at $0^{\circ}$ angle of attack and a freestream dynamic pressure of $30.7 \mathrm{psf}$, in order to observe the effects of applying lower viscosity oil to areas were separation does not occur. Figure 34 shows the regions of different oil viscosity and the resulting fringes from the control test.

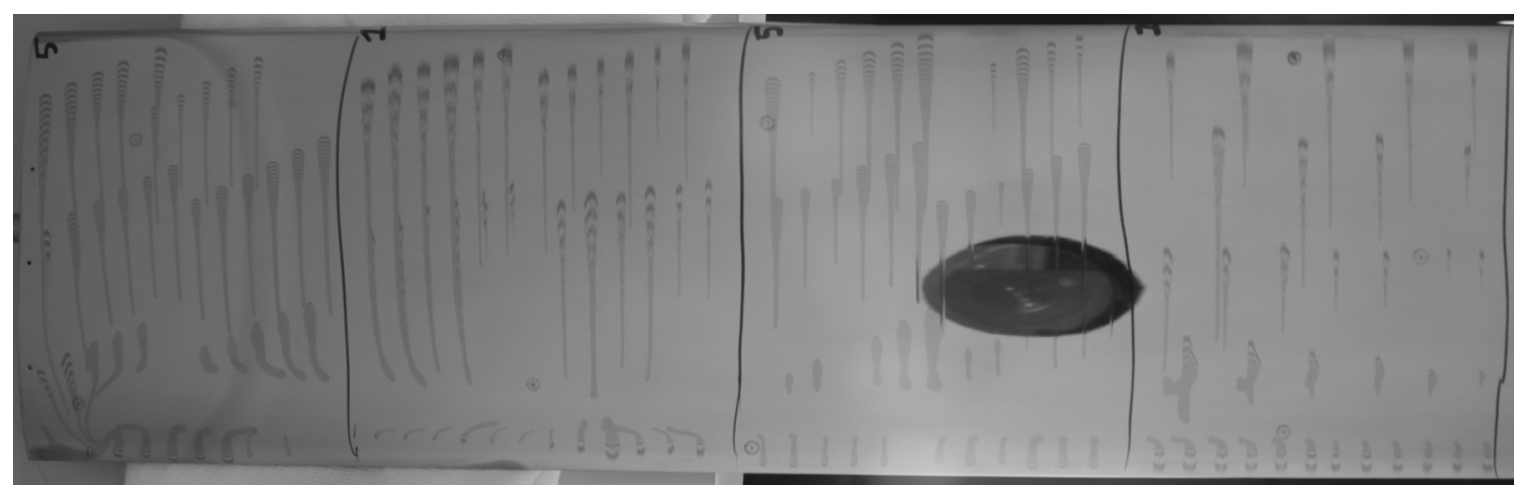

Figure 34. Control experiment for oils of differing viscosity (5 indicating 500cSt and 1 indicated 100cSt) conducted at $\alpha$ of $0^{\circ}$ and a $q_{\infty}$ of $30.7 \mathrm{psf}$.

For cases where identical flow conditions exist and oil viscosities of significant difference are being tested, lower viscosity oil fringes degenerate by the time the higher viscosity oil fringes begin to develop. The $100 \mathrm{cSt}$ fringes at the leading edge have higher noise in their intensity levels, increasing the regression uncertainty, while the $500 \mathrm{cSt}$ 
leading edge fringes are appropriately spaced. However, in regions of lower skin friction, the $500 \mathrm{cSt}$ had insufficient time to develop while the $100 \mathrm{cSt}$ fringes are appropriately spaced.

In order to accurately capture results for the separated regions, subsequent testing using multiple oils was conducted for angles of attack of $5.0^{\circ}, 7.5^{\circ}$, and $10.0^{\circ}$ for a freestream dynamic pressure of $30.7 \mathrm{psf}$. During these tests, $500 \mathrm{cSt}$ oil was used in the attached region from the leading edge to nearly $30 \%$ chord while $100 \mathrm{cSt}$ was used in the separated region from $30 \%$ chord to the trailing edge. Results from the $5.0^{\circ}$ test can be seen in Figure 35.

Figure 35 indicates that the flow actually remained attached despite the angle of attack. A laminar separation bubble can be seen at the leading edge, followed by a region of turbulent flow. Flow separation can be seen at the trailing edge; however velocity in the separated region was too low to create fringes in the $100 \mathrm{cSt}$ oil.

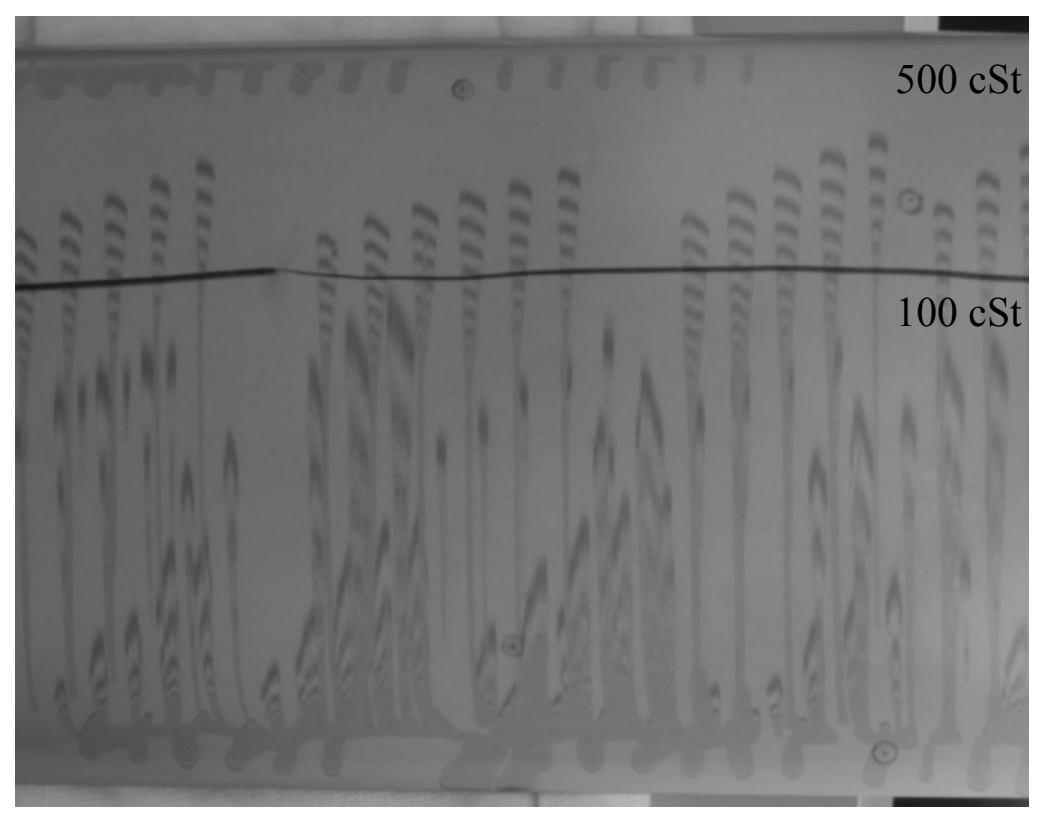

Figure 35. Results of $5.0^{\circ}$ angle of attack test. A sharpie line at $30 \%$ chord differentiates the $100 \mathrm{cSt}$ and $500 \mathrm{cSt}$ oil. 
The test was repeated at an increased angle of attack of $10.0^{\circ}$, resulting in separated flow over the entire upper surface. The last attempt to accurately collect data from the application of multiple oil viscosities was conducted at an angle of attack of $7.5^{\circ}$. Results of this test were similar to the $5.0^{\circ}$ test in that a small laminar separation bubble occurred at the leading edge, tripping the rest of the upper surface to turbulent flow, followed by a separated trailing edge where velocity was too low to create fringes.

Simultaneous use of oils of differing viscosity will be helpful in efficiently capturing data from the separated, turbulent, and varying velocity flow regions during the AMELIA testing. Tests conducted on the two foot blended wing section provided the following guidelines when applying oils of differing viscosity: The accurate prediction of differing flow regions is critical to successfully testing with multiple oils, oil viscosity regions must be clearly distinguished in order for oil viscosity to be identified during data acquisition, and oil droplets must be placed such that resulting fringes do not intersect.

\section{Experimental Validation}

It was desired to validate the FISF technique applied to the wing section against a known skin friction to ensure the technique was being applied correctly. Due to the small Reynolds number and laminar flow, the wing boundary layer is quite thin, approximately 0.03 inches at a point 2.9 inches from the leading edge. A Stanton tube is the ideal skin friction measurement device for these conditions. However, the model is not instrumented with static pressure ports. A Preston tube was the next available choice. For Preston tubes to be accurate, the total pressure probe diameter must be much smaller than the boundary layer. ${ }^{47}$ A Preston Tube Data System ${ }^{48}$ (PTDS) loaned from Russell Westphal was utilized to make skin friction measurements on the wing. A total pressure probe diameter of 0.025 inches was chosen, as smaller diameters yield poor pressure 
response, introducing additional measurement inaccuracies. Utilizing Preston's calibration for turbulent skin friction measurements, the skin friction coefficient was calculated to be 0.00128 at a freestream dynamic pressure of 30.8 psf. Preston's calibration was chosen since the trailing edge of the airfoil was shown to have turbulent airflow for the validation case shown here. The Preston tube accuracy was calculated to be $2.3 \%$ but is known to have a rough $5 \%$ accuracy. ${ }^{\S}$ Figure 36 illustrates interpolated skin friction data points at a constant chord location of 13.55 inches. The data matches well near the location of the Preston tube, with the nearest data point having $1.8 \%$ difference. Since the data points are interpolated along a particular location, the dataset has a large variation. This test validates the methodology being utilized in the current testing.

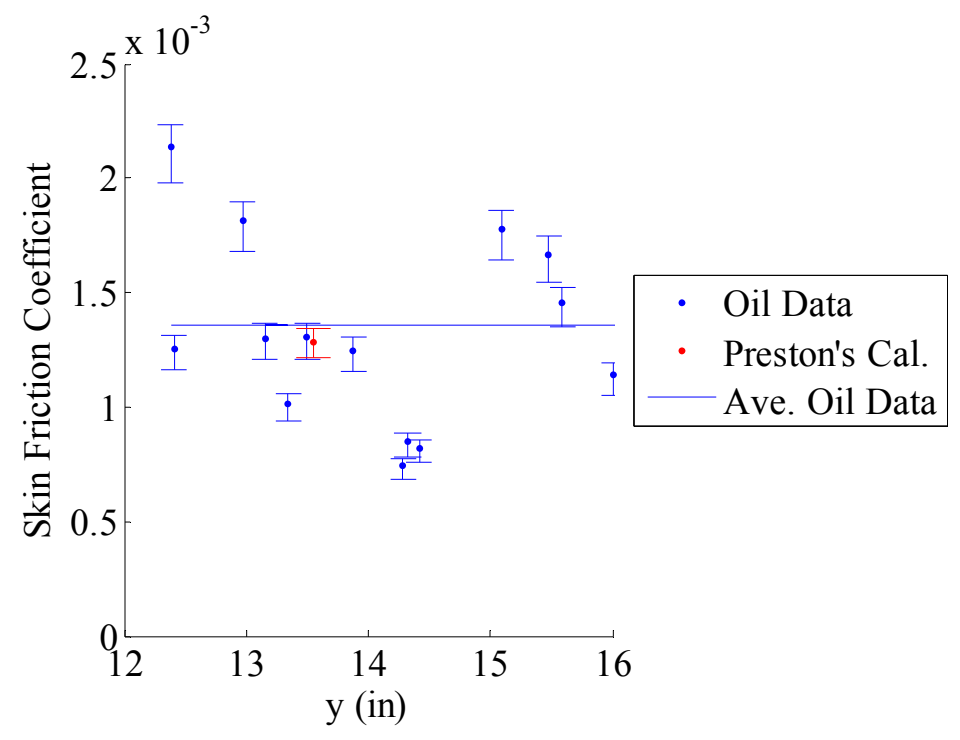

Figure 36. Interpolated oil data points at $x / c$ location of 0.82 illustrating a mean value comparable with Preston's skin friction calibration.

An attempt to increase the accuracy of the measurement was made by decreasing the freestream velocity to increase the boundary layer thickness. Despite utilizing $100 \mathrm{cSt}$ oil,

$\S$ Westphal, R. Private communication., July 2010. 
fringes failed to develop in the area of interest. At higher freestream velocities (110 fps to $150 \mathrm{fps}$ ), the boundary layer does not thin appreciably so the case for a freestream velocity of $163 \mathrm{fps}$ is shown.

\section{Experimental Uncertainty Summary}

The main source of uncertainty previously considered was due to oil viscosity variations with temperature. However, as shown in Table 4, additional errors must be accounted for when utilizing Zilliac's processing software. Human repeatability is on the same order as calibrated oil, being quite significant in the overall uncertainty. Photogrammetry uncertainty for this particular test was found to be high. However with accurately placed fiducials, photogrammetry error should be negligible. The experiment was found to be repeatable except due to variations in transition. A range of acceptable focus lengths were identified for a $28 \mathrm{~mm}$ zoom. Data taken from blurry fringes is unacceptable. Utilizing HDR to enhance images with extreme exposure variations is discouraged due to an increase in image noise. Lastly, the filters were found to have insignificant influence on the skin friction coefficients. 
Table 4. Summary of additional error sources investigated.

Error Source

Human Repeatability

Photogrammetry

Experimental Repeatability

Camera Focus

High Dynamic Range

Filter Variation

\section{Uncertainty range}

$\pm 1.0 \%$ to $\pm 2.3 \%$ of $\Delta s$

$\pm 1.4^{\circ}$ to $\pm 2.0^{\circ}$ of $\theta$

$-6.9 \%$ to $+3.8 \%$ of $\Delta s$

very small

very small with clear fringes

up to $\pm 25 \%$ of $c_{f}$

$0 \%$ to $\pm 3 \%$ of $c_{f}$ 


\section{Chapter III: Validation of a Micro Flow Measurement Device}

\section{Introduction}

Boundary layer measurement devices have improved over the years, continually yielding data closer to the wall surface. Measurement options include hot wire anemometers, Pitot probes, and Pitot-static probes. At extremely close wall positions, hot wires and hot films incur inaccuracies from the rapid change in heat convection, thereby limiting their usefulness. The physical size of total pressure probes has limited the nearest wall velocity measurement to 0.010 inches. ${ }^{49}$ Pitot-static probes will never achieve data closer than Pitot probes due to their dual tube construction.

In 2001, Hwang ${ }^{49}$ developed a thermocouple boundary layer rake which has the capability to measure 0.0025 inches from the surface, four times closer than any state-ofthe-art measurement before. This device is made up of a quartz substrate which protrudes into the flow, a platinum and gold thermocouple mask, and an aluminum base which holds the substrate in place. Beneath the base surface, wires lead to a data acquisition system. From the base to the top of the strut, there are several pairs of thermocouples. The device functions based on the theory that for a given height above the base, the velocity is equal to the velocity of the flow as if there were no strut present.

At the center of the strut, there is a platinum loop which is heated to an arbitrary temperature above ambient. As air flows over the loop, the air downstream is heated. The voltage difference between the upstream and downstream thermocouples is then related to known velocity values obtained from a total pressure rake setup next to the 
thermocouple rake at exactly the same axial position. A calibration is required at every Reynolds number to be tested since not all the upstream voltages could be maintained at the same voltage. Further information can be found in Hwang. ${ }^{49}$

Upon discussions with Gustave Fralick, one of the thermocouple rake's designers, the use of a Cross Correlation Rake (CCR) was suggested. The CCR operates on time of flight theory and does not require a lengthy in situ calibration since raw voltage differentials along the loop are being compared. To date, a CCR proof of concept has only been validated for shop air being blown over a prototype. ${ }^{50}$ The CCR therefore requires validation before being applied to a costly wind tunnel test.

Once validated, the CCR will be implemented on the AMELIA test in summer of $2011 .^{8}$ The CCR is a part of the validation effort to acquire velocity profiles above the blown flap. This region is of particular interest because of the jet's boundary layer as well as the shear layer acting above the flap.

The design, construction, and testing of a micro boundary layer measurement device designed for transonic speeds will be completed. The substrate will be constructed at $\mathrm{Cal}$ Poly, with the platinum applied to the substrate at NASA Glenn. Once constructed, the device will be validated subsonically on a flat plate.

\section{CCR Operational Theory}

The theory behind the CCR originates from the use of time of flight to measure velocity. If the time required for an object to travel from one location to another is known, the velocity of that object can be computed.

The CCR contains a conductive platinum loop located at the center of the substrate, shown in Figure 37. This loop is heated to a temperature above ambient conditions. 
Instantaneous voltage differences on the loop's leading and trailing edge are measured by voltage taps along the loop. Upstream taps in Figure 37 are A and B while downstream taps are labeled $\mathrm{C}$ and $\mathrm{D}$. The differential voltage between tap A and B is amplified before being recorded by the data acquisition system.

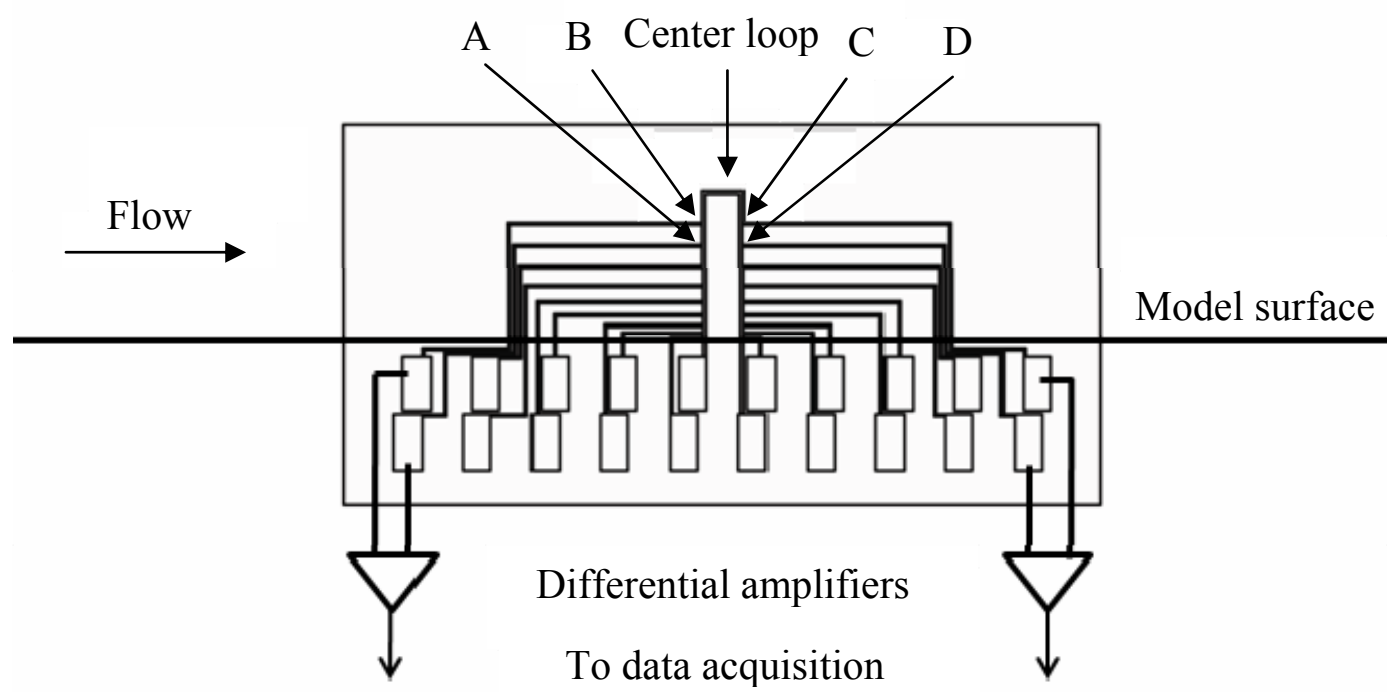

Figure 37. Diagram of the CCR operational methodology with voltage taps shown at $A$ and B locations.

Voltage fluctuations in the loop are caused by fluctuations in the local resistance due to flow variations. The resistance relates to the loop temperature, which relates directly to velocity at that location. ${ }^{50}$ As fluctuating fluid flows by the upstream loop, small changes in the voltage can be measured. The same fluid then flows over the downstream loop, creating similar voltage changes. Utilizing a cross correlation function from MATLAB (discussed further below), ${ }^{51}$ the time between voltage fluctuations on the upstream and downstream voltage taps can be calculated. Since the distance between the upstream and downstream edges of the loop is known, the flow velocity can be calculated.

Cross correlation is the measure of similarity between two random functions. ${ }^{52} \mathrm{~A}$ delay between functions can be computed, occurring where the functions are most 
similar. This is completed by integrating the product of the two functions, one of which is offset by some time step, $\tau$. The time step for which the integral is a maximum corresponds to the lag of the second function. This is represented by Equation (10)

$$
r(\tau)=\frac{1}{N} \sum_{t=0}^{N-1} g(t) d(t+\tau)
$$

where $r$ is the sum of the product of the functions, $g$ is a function at time $t$, and $d$ is an offset function at time $t$ plus some delay $\tau$. The delay ranges from zero to the complete duration of the dataset. Figure 38 illustrates two random, identical functions, one offset five seconds from the other while Figure 39 shows MATLAB's cross correlation function, with a peak occurring at a five second offset. It should be noted that MATLAB's cross correlation function computes the $r$ function at $2 N-1$ points, calculating positive and negative delays. In theory, this allows the CCR to indicate flow reversal.

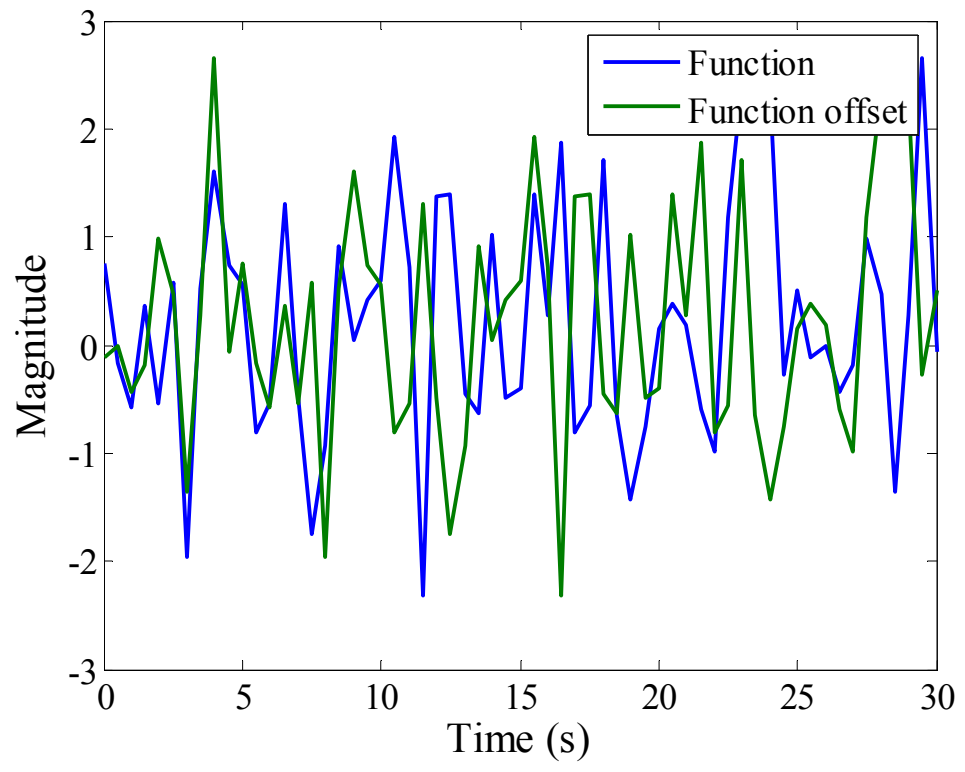

Figure 38. Example of a random function plotted against the same function offset by five seconds. 


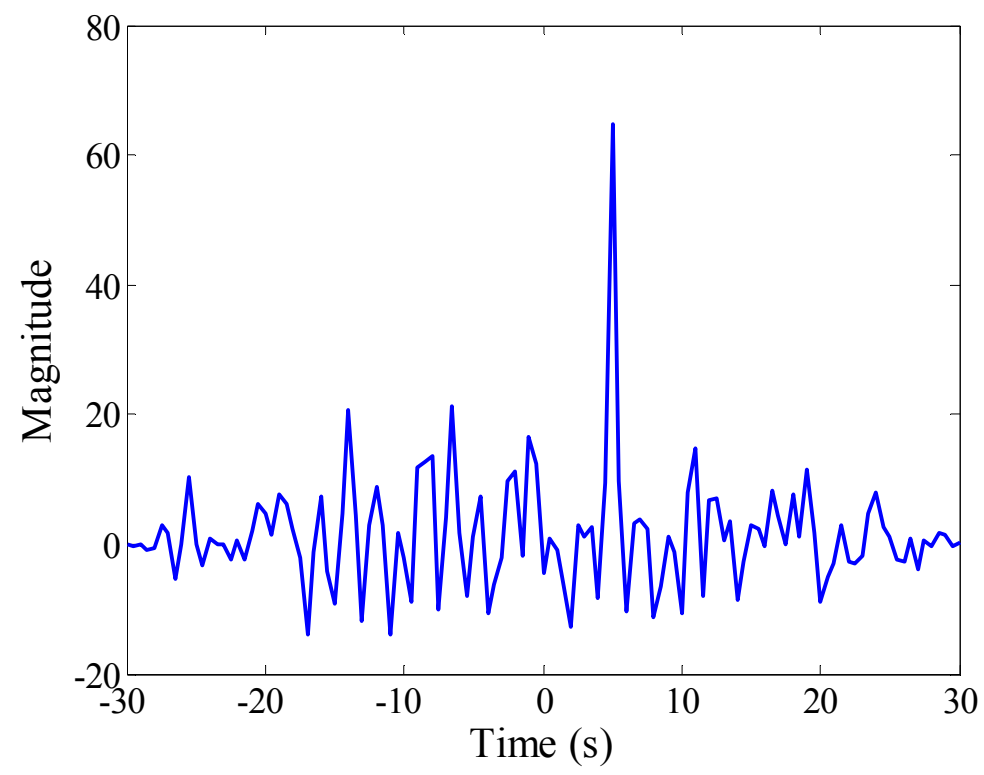

Figure 39. The function delay of the example random function shown at left, with a clear correlation peak at five seconds.

\section{CCR Design and Fabrication}

The CCR was designed for application on the AMELIA flap as shown in Figure 40. The design and application to AMELIA drove the various requirements for the CCR, as discussed below.

\section{Platinum Mask}

The platinum mask design is defined by the desired locations of the velocity measurements. Its specifications are also determined by the data acquisition rate and wiring requirements within the flap. The mask layout is shown in Figure 41 and is manufactured to $10^{-5}$ inch accuracy. Velocity measurements on the rake range from 0.00400 to 0.13594 inches from the surface, which was chosen based on preliminary CFD solutions, ${ }^{7}$ shown in Figure 40. A total of eight velocity measurements are made, requiring nine voltage taps. This number was chosen due to finite data acquisition channels and limited wiring space. The voltage tap leads are 0.003 inches wide while the center loop is 0.005 inches wide. The effective velocity measurement location is assumed 
to be at the center of the voltage taps. A hyperbolic spacing function was utilized to space the voltage taps to better resolve the jet boundary layer measurements. ${ }^{53}$ The first tap is at the level of the flap surface with the second defined to be 0.008 inches above the surface. From there, the tap locations are defined by the hyperbolic spacing function and the number of desired taps. Table 5 summarizes the tap and velocity measurement locations.

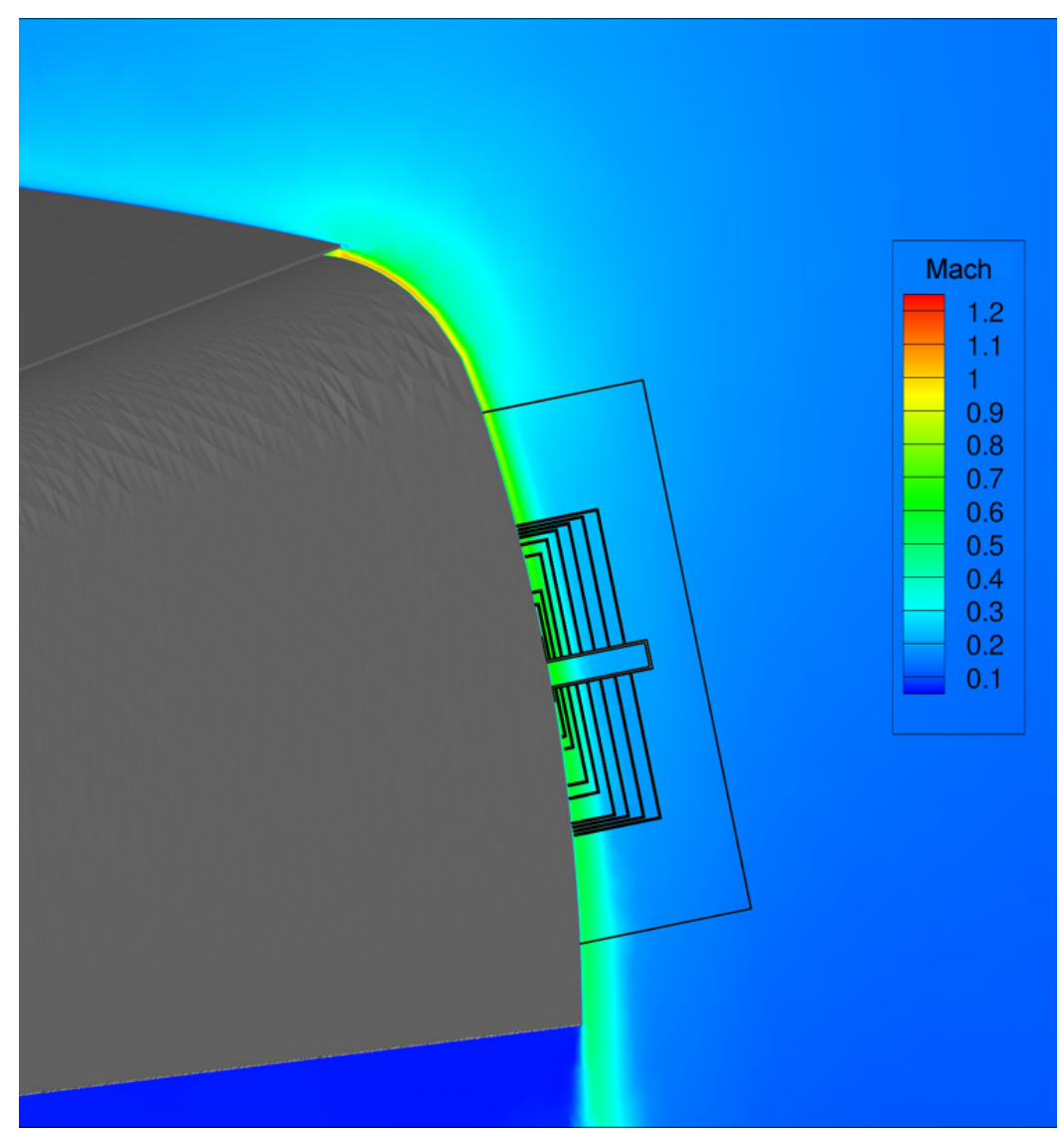

Figure 40. CFD solution of AMELIA illustrating a plane of contours of Mach number above the flap. The CCR platinum mask is superimposed over the solution. Conditions are at STP, $\alpha$ of $0^{\circ}$, flap $60^{\circ}$, and $M$ of $0.07 .^{7}$ 


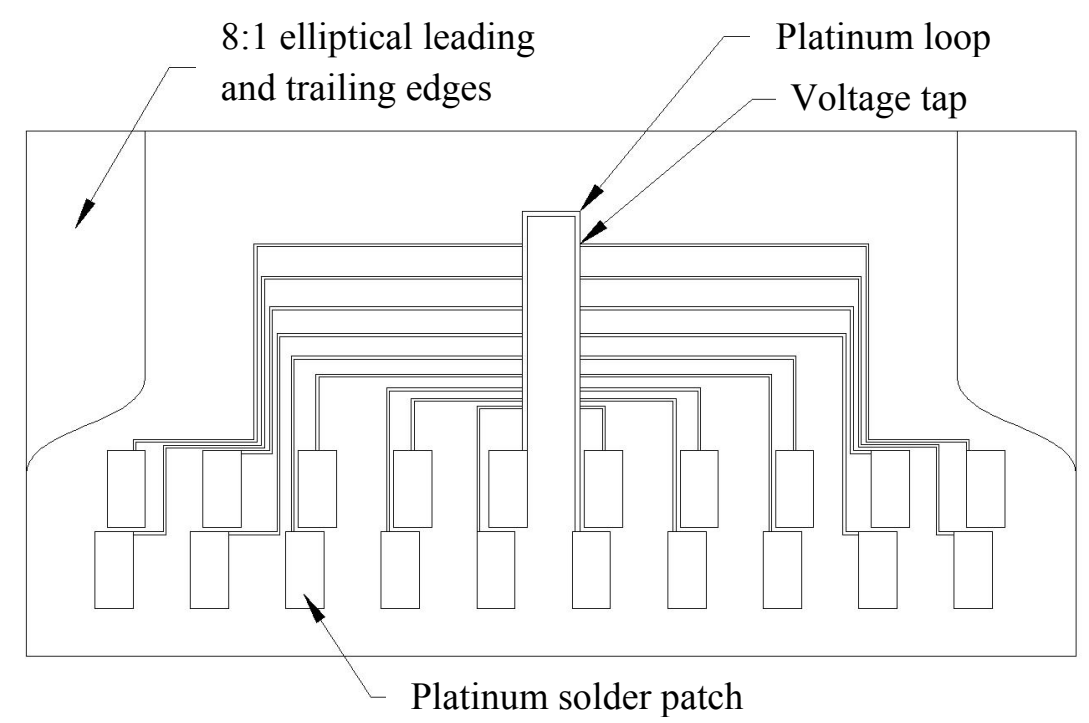

Figure 41. Schematic of CCR illustrating platinum mask on the quartz substrate and elliptical leading edges.

Table 5. Summary of voltage tap and velocity locations.

\begin{tabular}{cc}
$\begin{array}{c}\text { Tap Location } \\
\text { (in) }\end{array}$ & $\begin{array}{c}\text { Velocity Location } \\
\text { (in) }\end{array}$ \\
\hline 0.00000 & 0.00400 \\
0.00800 & 0.01345 \\
0.01890 & 0.02616 \\
0.03343 & 0.04285 \\
0.05227 & 0.06405 \\
0.07584 & 0.08993 \\
0.10402 & 0.11998 \\
0.13594 & 0.15297 \\
0.17000 & \\
\hline
\end{tabular}

The platinum loop spacing was determined by a trade between surface curvature accuracy and data acquisition rate. Ideally, the loop edges would be infinitely close to one another. However, the required data rate could not be achieved (discussed further in Data Acquisition). A spacing of 0.050 inches between the inner edges on the platinum loop 
was deemed acceptable in terms of surface curvature and required data acquisition rate. The platinum loop, like a CFD mesh, makes a linear approximation to the flap surface curvature. The maximum inaccuracy perpendicular to the linear approximation made by the platinum loop is 0.000326 inches, yielding a surface curvature resolved to $0.65 \%$. This surface resolution is represented in Figure 42.

There are 20 platinum solder patches at the bottom of the substrate allowing for a clean area to solder wires which does not disturb the flow. Of the 20 patches, 18 lead to voltage taps (nine on each side) with the remaining two patches for applying voltage through the platinum loop. Standard 0.10 inch spacing was utilized to ensure sufficient area for soldering. These locations are represented by the white rectangles at the bottom of Figure 41. Due to the limited substrate length, the platinum leads will be offset from one another, allowing multiple wires to be soldered in a smaller area.

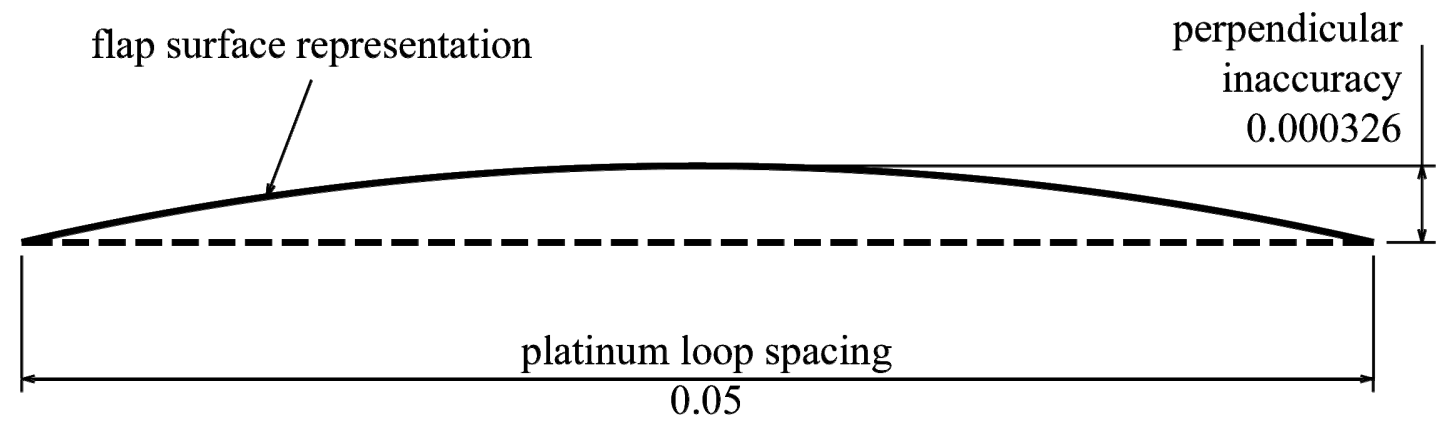

Figure 42. A schematic (skewed for clarity) of platinum loop resolution, with dimensions in inches.

The platinum mask is only a few microns thick, having little disturbing effect to the flow over the surface. The platinum is applied via a photolithographic process which NASA Glenn specifically developed for the thermocouple boundary layer rake. ${ }^{49}$ Plating issues arose due to scratches on the substrate surface causing discontinuities in the 
platinum mask. The scratches were caused by the original substrate manufacturing process discussed in Quartz Substrate.

Care must be taken when heating is applied to the platinum. Quartz has a coefficient of expansion of $0.55 \cdot 10^{-9} /{ }^{\circ} \mathrm{C}$ while pure platinum is much higher at $8.9 \cdot 10^{-6} /{ }^{\circ} \mathrm{C} .{ }^{54,55}$ Due to quartz's insulative properties, the platinum would heat much faster than the quartz, leading to further differential in the expansion. While prototyping the CCR wiring, varying voltages were applied to the platinum loop. Near 15 volts, the platinum loop peeled off of the quartz substrate. This corresponds to 22.5 watts released across the platinum loop. If flow were applied to the platinum, the convective cooling could possibly make the high power output acceptable. In testing, three volts across the platinum loop was found to yield sufficient heating for cross correlation functionality.

\section{Quartz Substrate}

Quartz was chosen as the substrate material for its insulative and low thermal expansion properties compared to glass. The substrate is of constant thickness and therefore analogous to a flat plate. In order to make accurate velocity measurements, there must be a large region on the substrate where the pressure coefficient is zero. An elliptical leading edge was utilized to encourage flow uniformity over the substrate. Since the jet flow from the slot on AMELIA will be at transonic velocities, transonic flat plate design was investigated to determine an appropriate ratio for major to minor axis on the leading edge. Saric ${ }^{56}$ used a 14:1 ratio for a flat plate in the National Transonic Facility. This would be ideal, but impractical due to the scale of the substrate. The leading edge and trailing edges would have a 0.294 inch long blend, which together would be over half the length of the substrate. Though there is a smaller suction peak, the constant thickness portion of the airfoil would have insufficient length. The smallest ratio used in other 
research was found to be $5: 1 .^{57}$ A compromise of $8: 1$ was instead chosen to maximize the constant pressure region over the substrate while avoiding a large suction peak. Trailing edge corner separation is expected, ${ }^{58}$ but should not adversely affect the data.

To ensure constant pressure over the central portion of the substrate surface, the substrate needed to be as long as possible. However, it could not be too near to the trailing edge slot on AMELIA to avoid blockage, or the trailing edge due to manufacturing limitations. The resulting length was 1.10 inches. The height was based upon the desired height above the flap to measure velocity profiles and required space below the flap to solder wires to. The total height of the rake is 0.55 inches, with the top half above the flap and the remaining lower half below the flap. Quartz microscope slides from Ted Pella, Inc. were utilized as the substrate and have a nominal thickness of 0.042 inches.

The substrate leading edge was shaped with an aluminum guide block. The aluminum block had half of the elliptical leading edge profile machined into it. The quartz substrate sat in the aluminum block while the extraneous quartz material was removed by sanding, illustrated in Figure 43. The substrate was then flipped over and the remaining side was sanded. This was repeated for the trailing edge. Scratches on the face of the substrate from sanding caused discontinuities in the platinum mask. Tape was placed over the flat portions to protect the substrate during manufacturing. However of five bases manufactured, none had fully successful platinum applications. New substrates will have the elliptical edges machined rather than sanded, but issues may arise from chipping on the substrate edge. To date, the best manufacturing process is still unknown. 


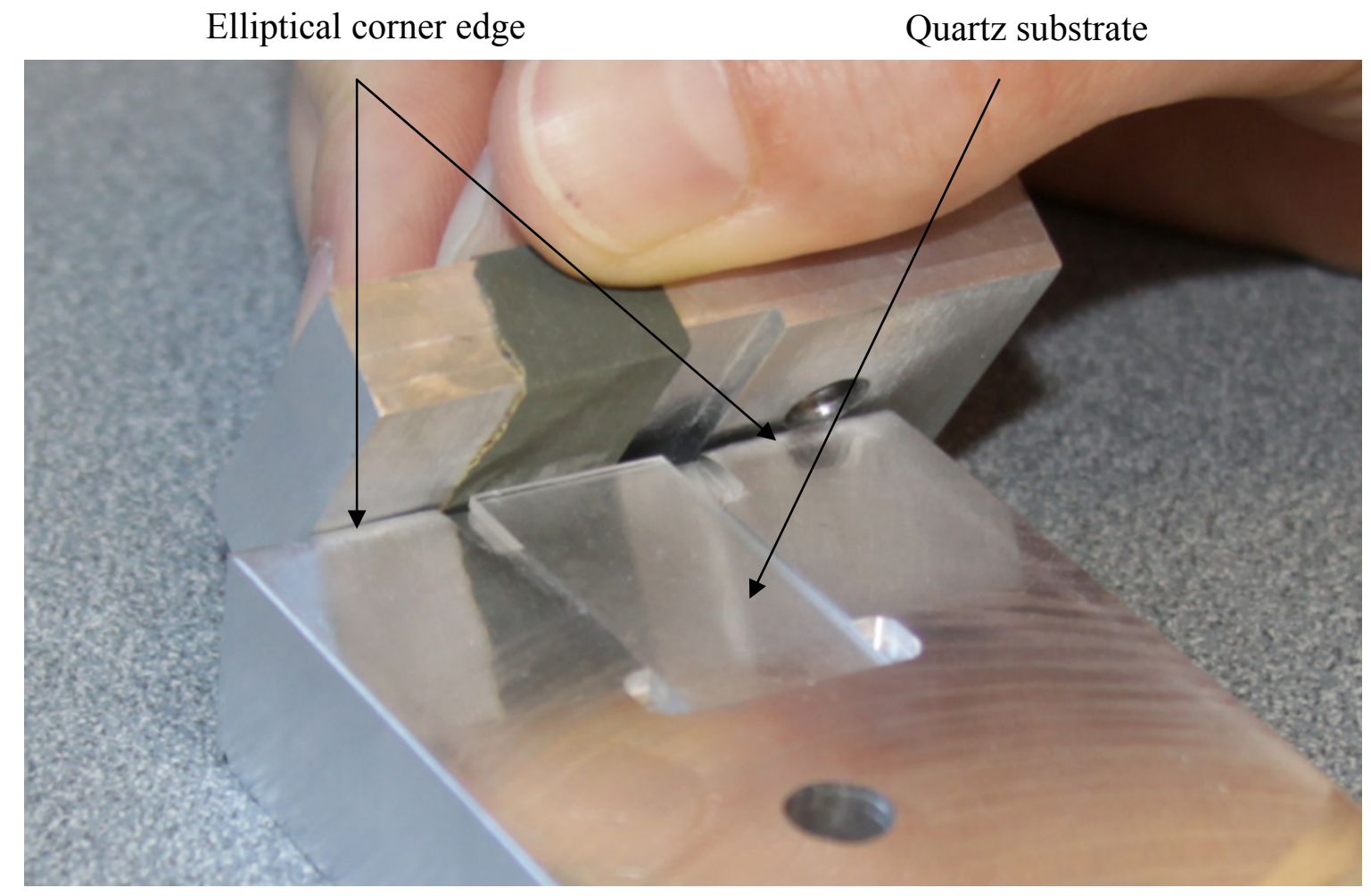

Figure 43. A photograph of a CCR substrate being shaped. The masking tape protecting the quartz substrate surface is omitted for clarity.

\section{Substrate Base and Flat Plate Insert}

The substrate will be held in place with an aluminum base, similar to NASA Glenn's thermocouple boundary layer rake. When the substrate is placed in the base, the platinum loop's vertical edges are perpendicular to the model surface. The substrate fits into the base and is wrapped with one mil Kapton ${ }^{\circledR}$ polyimide tape that will seal the interface between the base and the substrate ensuring a flush, smooth transition from the substrate to the base.

The base itself is composed of two asymmetric halves, shown in Figure 44. The halves join together with countersunk bolts on one side, allowing the substrate to be held in place by friction between the Kapton ${ }^{\circledR}$ and base. A CCR installed in the base is shown in Figure 45. The base slides into an insert for a flat plate. Bolts from underneath the 
insert hold the base in place, thus ensuring clean flow quality on the base surface. Also, o-rings placed between the base and insert allow for base height adjustments relative to the insert, illustrated in Figure 46. A complete installation is shown in Figure 47.

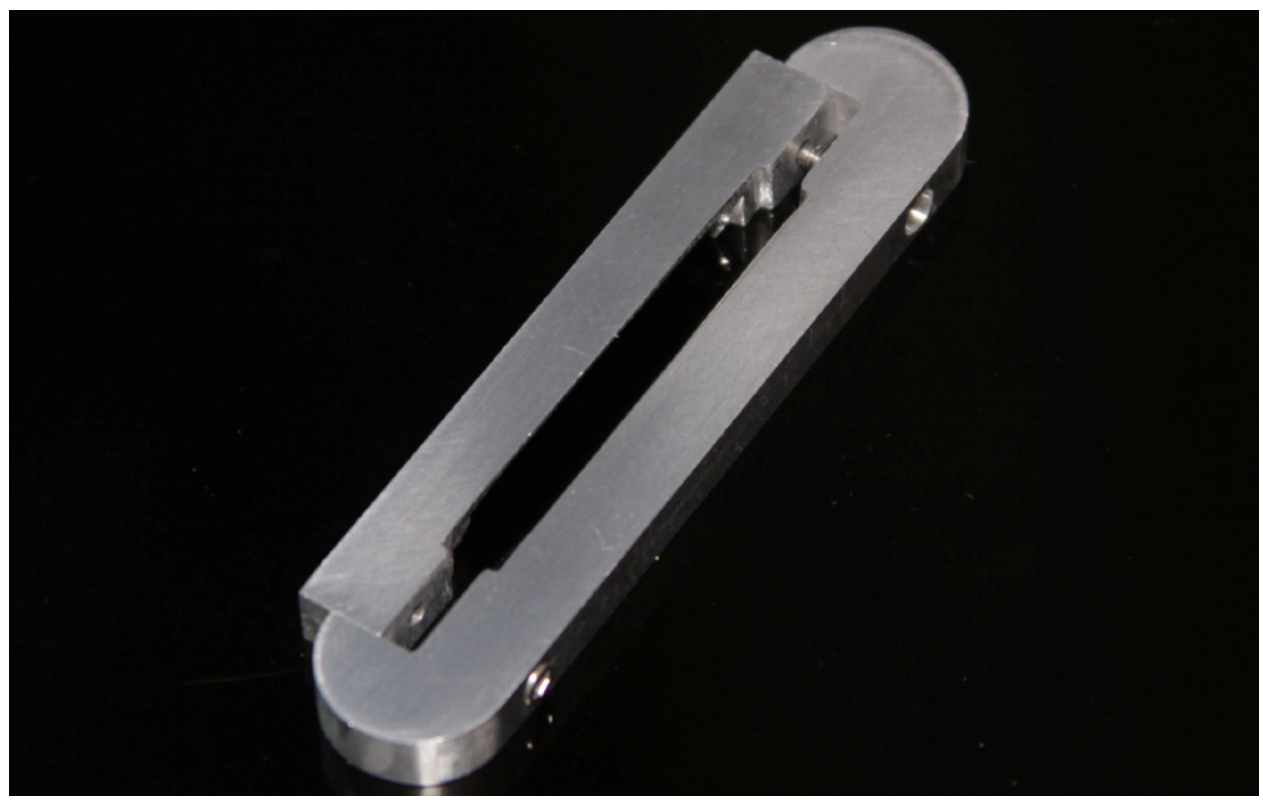

Figure 44. Image of rake base halves separated, illustrating where and how the substrate is held.

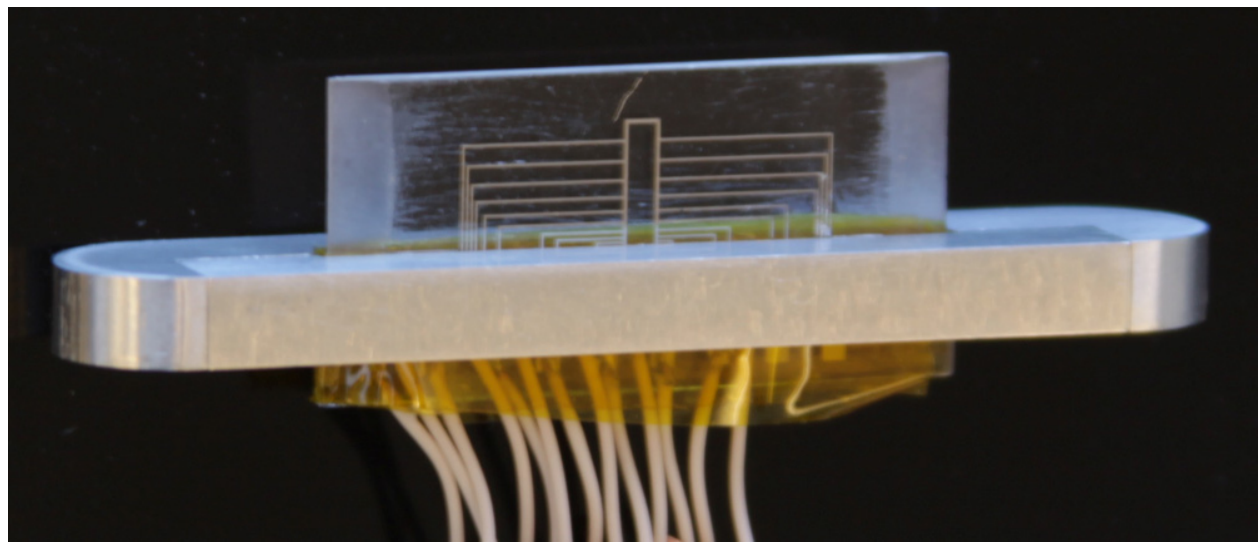

Figure 45. Photograph of CCR substrate and base with wiring shown leading out the bottom of the image. 


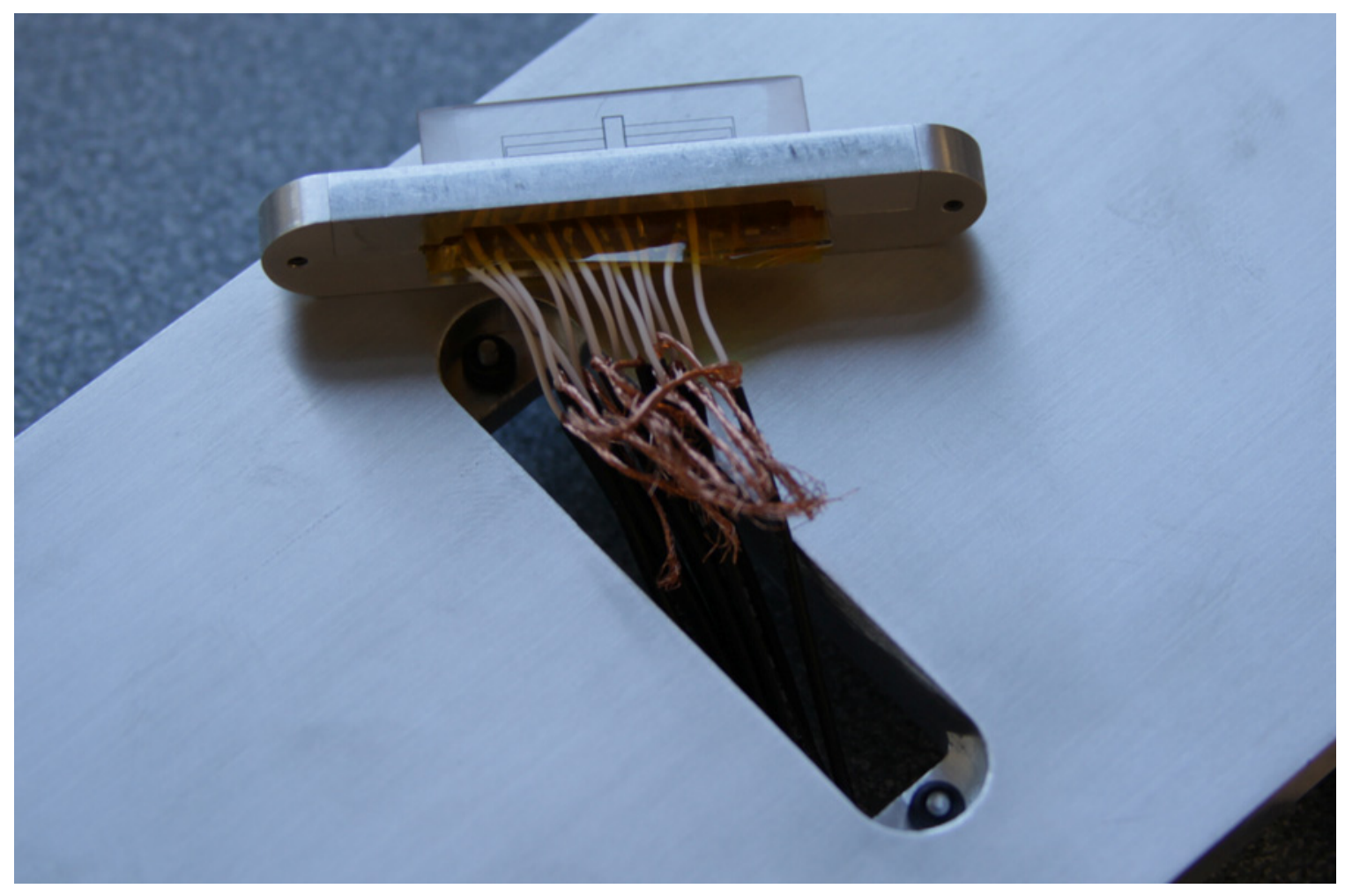

Figure 46. Image of CCR before installation onto the flat plate insert. One o-ring is visible in the lower right side of image which allows for base height adjustment.

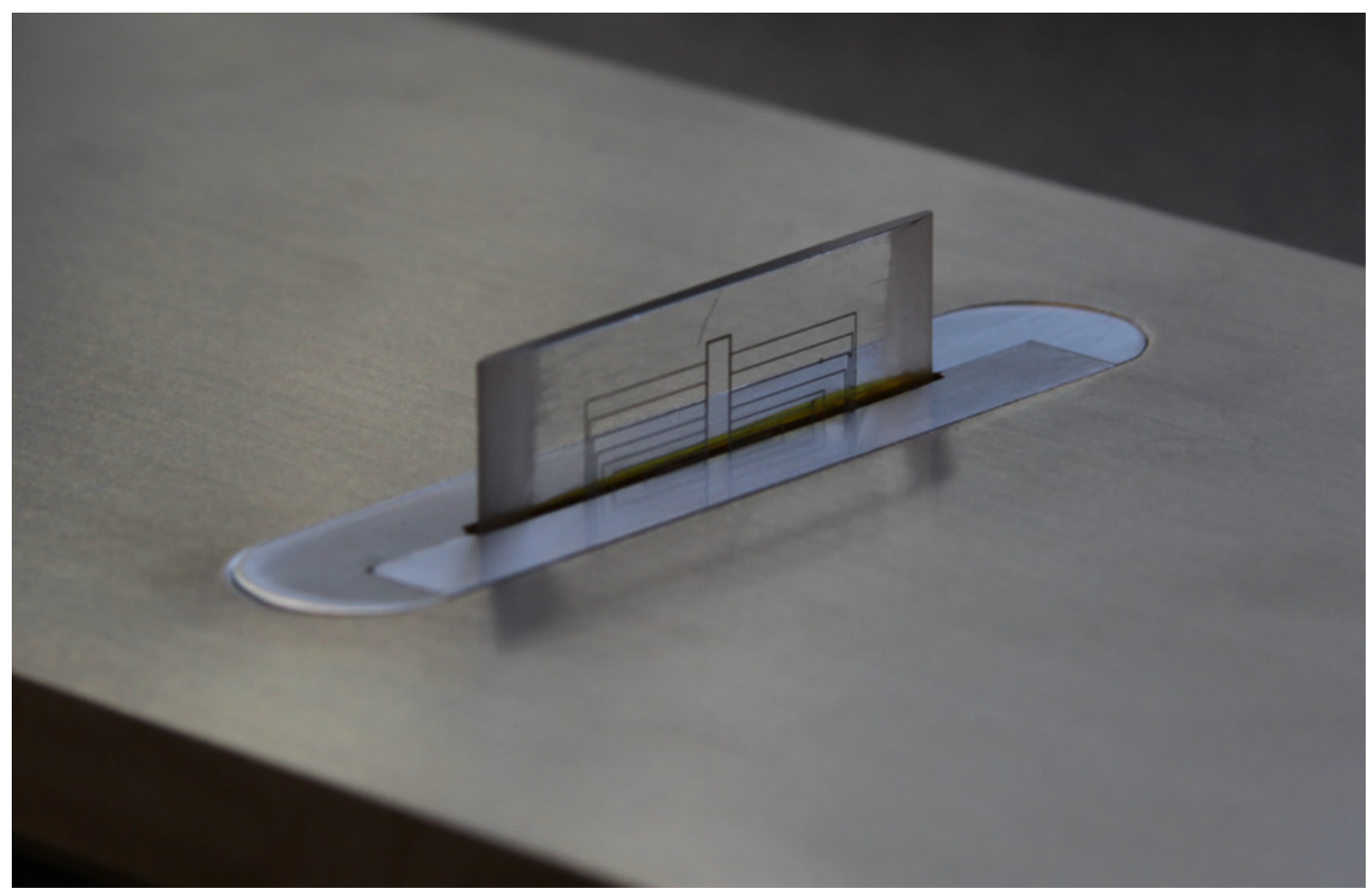

Figure 47. CCR installed onto flat plate insert. Kapton ${ }^{\circledR}$ tape on the non-platinum side is obscuring the lower voltage taps. 


\section{CCR Robustness}

Numerous issues arose with the robustness of the CCR and should be noted for future use. First, the quartz substrate was found to be more fragile than expected. Before sent to NASA Glenn, no cracks were present in the substrates. Upon receiving the returned substrates, multiple cracks and chips were present in the quartz. Small fractures may have occurred during substrate shaping which propagated during the manufacturing process completed at NASA Glenn. It is possible that the damage occurred during transport. Care must be taken while installing the substrate onto the CCR base. If the base is tightened too much, the quartz will crack.

Second, the platinum did not remain adhered to the quartz as well as desired. Multiple solder patches would detach during the handling of the CCR in everyday use. One detached over night directly after soldering. A likely explanation is that the platinum heating during soldering could weaken the bond between the platinum and quartz due to their differing material properties (described in Platinum Mask). Another possibility is that scratching on the quartz surface from sanding the edges caused poor platinum adhesion. Slightly roughening the quartz surface would increase the adhesion surface area, possibly improving the platinum-quartz bond.

Of the six substrates originally sent to Glenn, four were somewhat successfully plated. Each of the four had at least one, if not more, discontinuities along the voltage taps. Of the 20 solder patches on the first substrate, seven on the right side did not adhere well and quickly separated, leaving no remaining useful measurement locations (the left side did not lose any however). The second CCR's platinum loop was damaged from overheating the platinum, causing it to peel from the quartz. The loop peeling severed all the voltage taps along the loop. The third CCR was continually slipping from the CCR 
base, so additional Kapton ${ }^{\circledR}$ was applied to the substrate surface for an improved fit. When installed into the CCR base, the sides slightly bowed due to the additional Kapton ${ }^{\circledR}$. The CCR base and insert have a 0.001 inch tolerance. When the CCR base was installed into the insert, the CCR base bowing was forced inward, causing a crack to propagate along the substrate. In prior experiments, broken quartz held together well with cyanoacrylate. Therefore, an attempt to reattach the broken quartz half and solder the broken platinum leads was made. While removing the Kapton ${ }^{\circledR}$ tape, many usable taps were broken off, despite having successfully utilized the same removal method on previous CCRs. To avoid similar voltage tap damage, the back, non-sticky side of the Kapton ${ }^{\circledR}$ tape was applied over the fourth CCR voltage taps. While allowing for easy removal, there was less protection from wire movement on the individual taps. Many solder patches were removed during handling. The left side of the fourth CCR lost seven voltage tap locations while the right side only lost one. To protect the solder patches in the future, a nonconductive thermal paste or epoxy will be applied over the soldered wires and quartz. This should increase the robustness of the solder locations.

Due to the issues with the platinum solder patches loosing contact, only one data point was available to make readings from on the fourth CCR. The remaining taps were at 0.07584 inches and 0.13594 inches, yielding a velocity measurement at 0.10589 inches.

\section{Data Acquisition}

\section{Wiring}

Due to the limited space on the AMELIA flap, extremely small wires needed to be chosen. To reduce noise in the measurements, Mogami No. 32 AWG coaxial cable with a 0.0394 inch jacket outer diameter was utilized until there was sufficient space for 
shielded twisted pairs. The shielded twisted pair wiring has $12 \frac{1}{2}$ wire pairs with a single foil shield around the pairs as a whole. The wire is data and communication cable from McMaster-Carr. A 25 pin D sub connector was utilized on the flat plate test to transfer from the Mogami wiring to the shielded twisted pairs.

\section{Data Amplification}

The data is amplified using differential amplifiers, with setup shown in Figure 48. Differential amplifiers can be built with one operational amplifier (op amp) and resistors, two op amps and resistors, or three op amps and resistors (shown below). ${ }^{59}$ A three op amp with resistors setup was chosen since it has variable voltage gain control with a single resistor and good common mode rejection, allowing for poor matching of resistors. In the configuration shown in Figure 48, the gain is described by

$$
V_{\text {out }}=\frac{R_{1}}{R_{3}}\left(1+\frac{2 R_{2}}{R}\right)\left(V_{2+}-V_{1+}\right)
$$

where $V_{\text {out }}$ is the op amp output voltage, $R_{1}, R_{2}$, and $R_{3}$ are resistors, $R$ is a variable resistor, $V_{2+}$ is the high voltage, and $V_{l+}$ is the low voltage. In order to yield a three volt drop across the platinum loop, a $10 \Omega 10 \mathrm{~W}$ resistor was employed to control the voltage. A high power resistor was required due to the high current draw from the car battery. With three volts across the $10 \Omega$ platinum loop, voltage differences along the loop varied between $20 \mathrm{mV}$ to $80 \mathrm{mV}$. A standard current was 0.6 amps. Since the voltage varies according to position, each differential amplifier required individual design to yield an appropriate gain. 


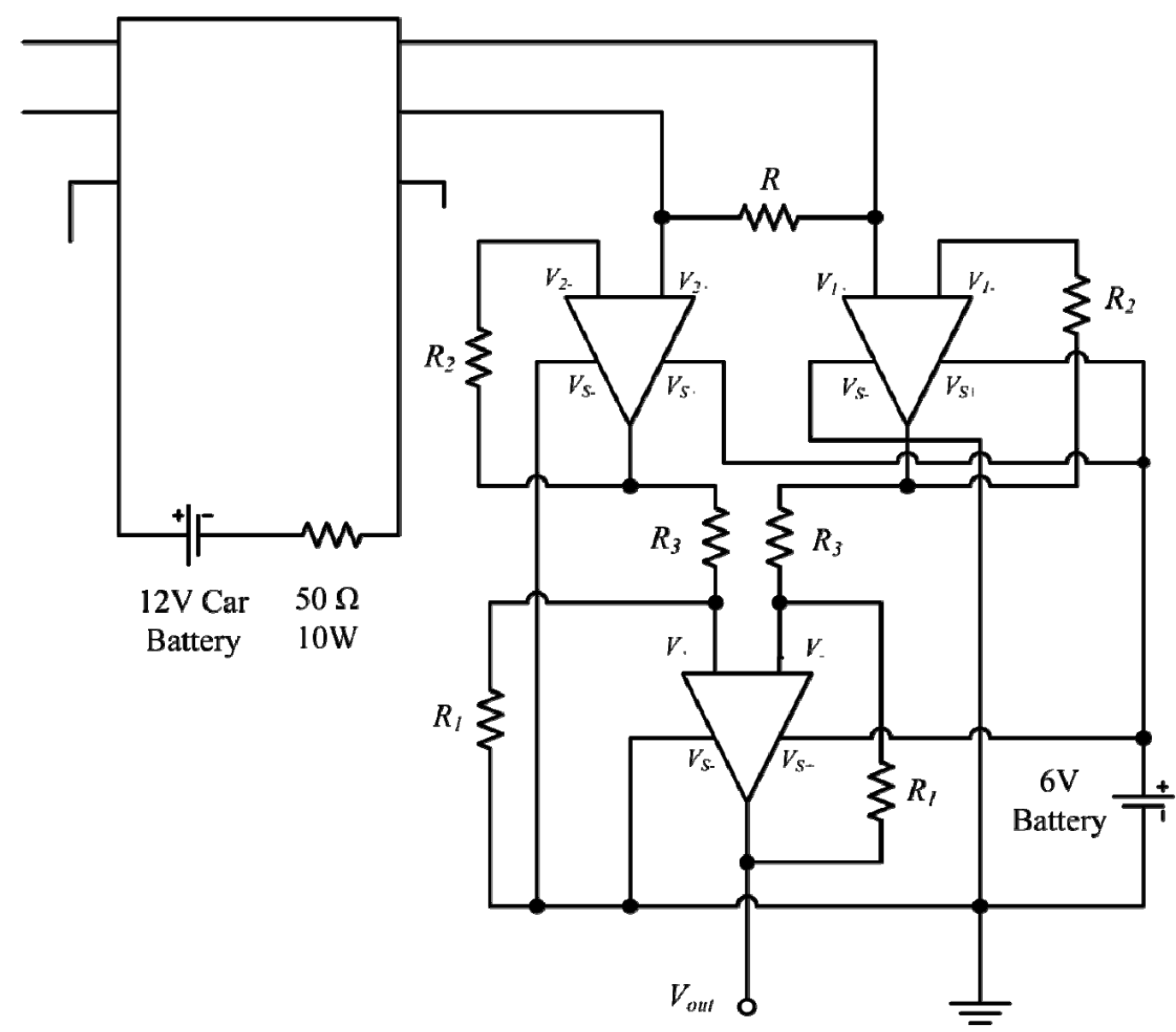

Figure 48. Electronic schematic of data acquisition setup. Only one voltage differential and six voltage taps are shown for clarity. A total of eight differential amplifiers were required.

The data card (discussed further below) has a variable range of $\pm 1.25 \mathrm{~V}$ to $\pm 10.00 \mathrm{~V}$. If a higher voltage is input into the data card, the card can be permanently damaged. A six volt battery was used as the op amp power supply, ensuring the input voltage would not be amplified beyond six volts. The variable range on the data card allows for a final gain to be applied to the voltage reading.

\section{Data Acquisition}

Two National Instruments PCI-6123 data cards will be utilized to acquire the voltage differentials. The data card has 16 bit resolution. Each has eight, $500 \mathrm{kHz}$ simultaneous sampling channels, significant in that there is a dedicated analog to digital converter for each channel. Simultaneous sampling is required to be able to correlate the data. If 
multiplexing is employed, there are inherent offsets in the time measurements which would yield incorrect correlations. The second card's timing will be controlled by the first to ensure simultaneous sampling between data cards. The required data rate was determined from Niquist sampling theorem which dictates that a minimum of two samples per period must be acquired to adequately sample the data. ${ }^{60}$ As mentioned above, the card has an input voltage range of $\pm 1.25 \mathrm{~V}$ to $\pm 10.00 \mathrm{~V}$. If a lower range is selected, the card's sensitivity is increased. At the $\pm 10.00 \mathrm{~V}$ range, the card's accuracy is $0.31 \mathrm{mV}$ while at the $\pm 1.25 \mathrm{~V}$ range, the accuracy is increased to $0.038 \mathrm{mV}$. All measurements were taken in the $\pm 5.00 \mathrm{~V}$ range, with an accuracy of $0.15 \mathrm{mV}$.

To minimize flow angularity effects, the platinum loop was spaced with 0.050 inches between the inner edges. The slot jet flow velocity is slightly less than Mach 1 and was used as the maximum design velocity. Assuming flow travelling at $1117 \mathrm{fps}$ (worst case scenario) with a spacing of 0.050 inches, a sampling frequency of $536,160 \mathrm{~Hz}$ is required. Since the flow decelerates rapidly from the slot to Mach 0.7, see Figure 40, a $500 \mathrm{kHz}$ sampling rate will be sufficient for AMELIA. For the flat plate test, 250,000 samples at $250 \mathrm{kHz}$ were utilized as the sample number and sample rate. By Niquist theory, if the maximum flow velocity is $150 \mathrm{fps}$, the minimum sampling rate is $72 \mathrm{kHz}$. Additional data is useful in making good correlations, so the data rate was doubled to roughly $125 \mathrm{kHz}$. When filtering is applied, cutoff frequencies cannot be applied above half the sample rate because of Niquist theory. To yield the desired $125 \mathrm{kHz}$ unfiltered, a $250 \mathrm{kHz}$ rate is required.

\section{Noise Considerations}

The initial amplified data was noisy with a $1.5 \mathrm{mV}$ variation in the reading. Originally, a conventional benchtop power supply was utilized as the voltage source 
rather than a car battery. However, it was found that the power supply added a minor amount of noise to the measurements. This noise added an additional correlation spike at a time delay of zero since it was read by both upstream and downstream locations at the same instance. Figure 48 shows that the car battery was used as a floating voltage source. It was determined that referencing the car battery to ground yielded no improvement in data quality or noise. A ground from the power supply (building ground) was compared against the data card ground. The data card ground is also grounded to the building ground, but it was thought that more noise would exist due to the ground running through the computer. However, there was no difference in measurement noise levels between the two grounds. For convenience, the data card ground was utilized. An attempted was made at attenuating the noise by applying aluminum foil shielding to all the cables and amplification system. This yielded no change however. The computer monitor was turned off during data acquisition, which caused no difference in the noise. Lastly, the car battery was directly attached to the data acquisition blocks. Measurements showed the same $\pm 1.5 \mathrm{mV}$ noise level. It was determined that despite the quoted accuracy of $0.31 \mathrm{mV}, \pm 1.5 \mathrm{mV}$ noise would be the baseline reading from the data cards.

\section{Subsonic Flat Plate Validation}

\section{Apparatus}

A two foot wide, 0.75 inch thick, elliptical leading edge flat plate was utilized in the Cal Poly Mechanical Engineering two foot by two foot wind tunnel to validate the operational ability of the CCR. The flat plate contains four inserts following the 3.5 inch leading edge. The CCR was installed on the fourth insert, with the platinum loop centered 15.00 inches from the plate leading edge. The flat plate allows four inserts to be installed, shown in Figure 49. The inserts are bolted into the flat plate from the bottom of the plate. 
There is a 0.05 inch gap between the insert and flat plate. O-rings in this gap allow for insert height adjustment, ensuring a flush installation. Beneath the inserts, there are multiple holes allowing for cabling to be led to the underside of the flat plate. The CCR cabling was directed towards the back of the test section and then out through the sting balance hole on the tunnel floor. This setup is illustrated in Figure 50.

A Boundary Layer Data System (BLDS) ${ }^{48}$ was implemented 15 inches from the flat plate leading edge to serve as a velocity validation. The BLDS contains a static pressure, dynamic pressure, and traversing total pressure probe shown in Figure 51. The traversing probe allows boundary layer profiles to be measured. The total pressure probe has a diameter of 0.032 inches. Figure 49 illustrates the installation of both the CCR and BLDS on the flat plate before testing.

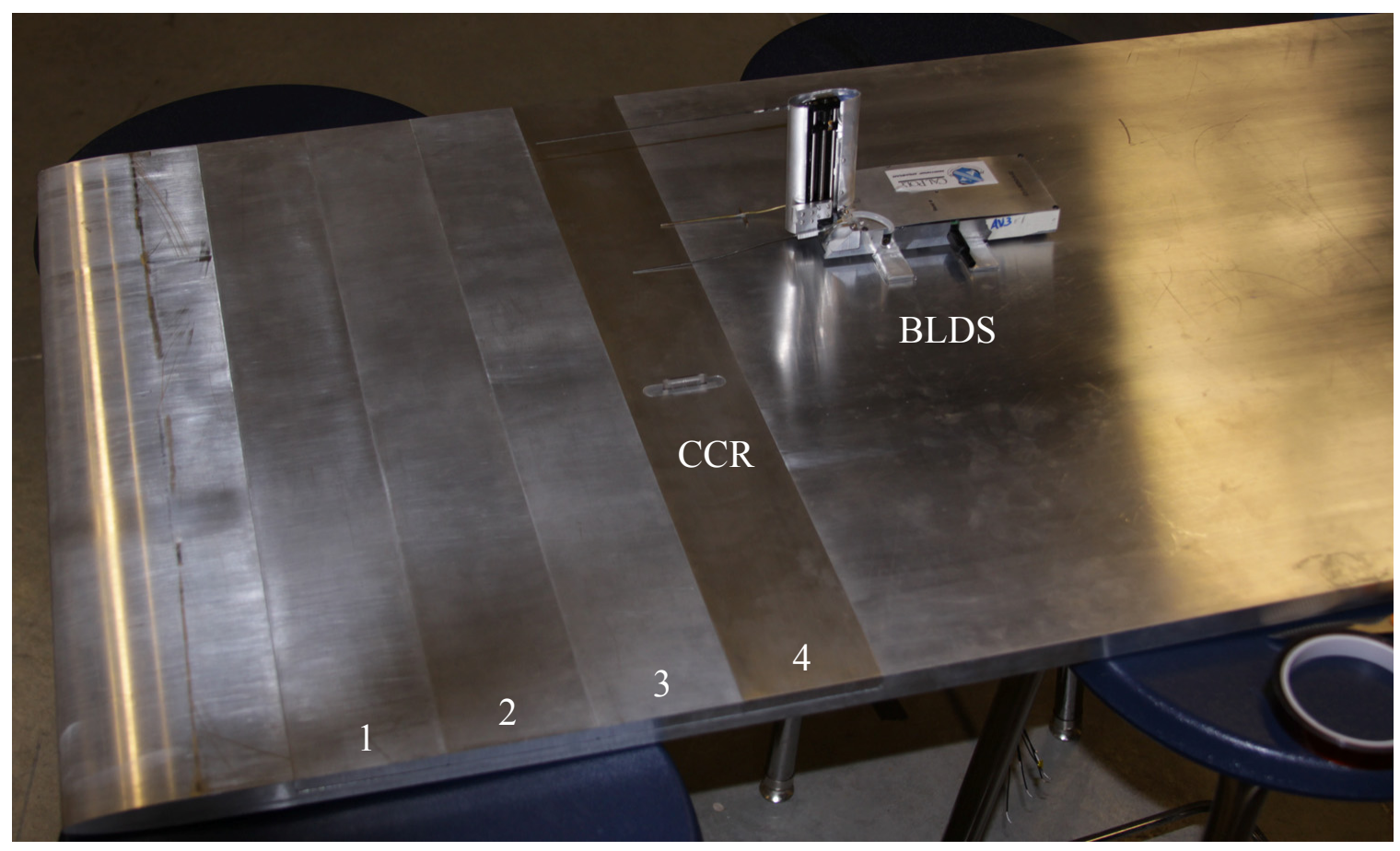

Figure 49. Image of the CCR implemented on the flat plate with BLDS in the background. The flat plate inserts are numbered 1 through 4, with the CCR on the fourth. 


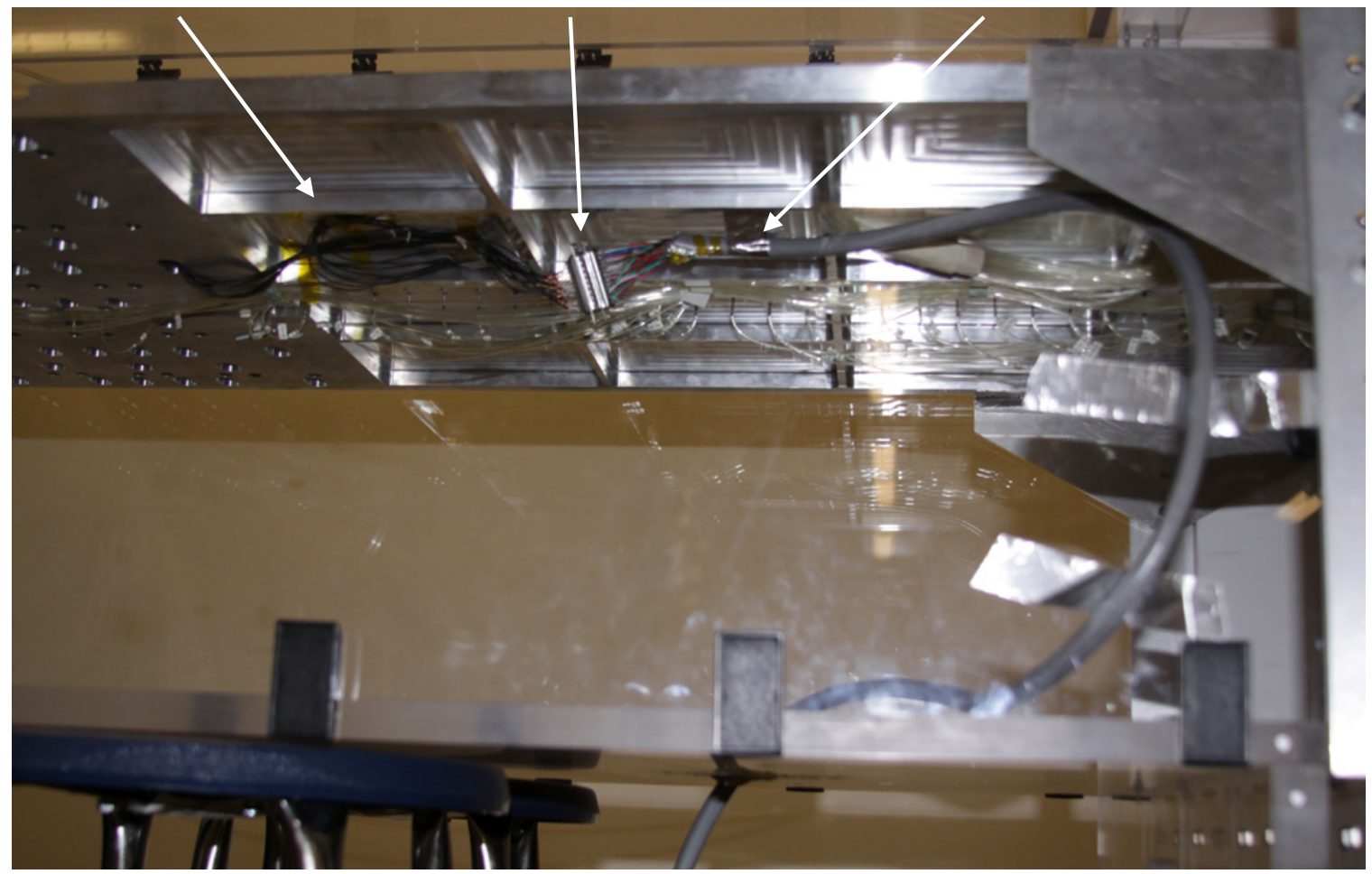

Figure 50. Underside of flat plate illustrating black Mogami cabling, D sub connectors, and grey shielded twisted pair data cable leading out of the tunnel floor.

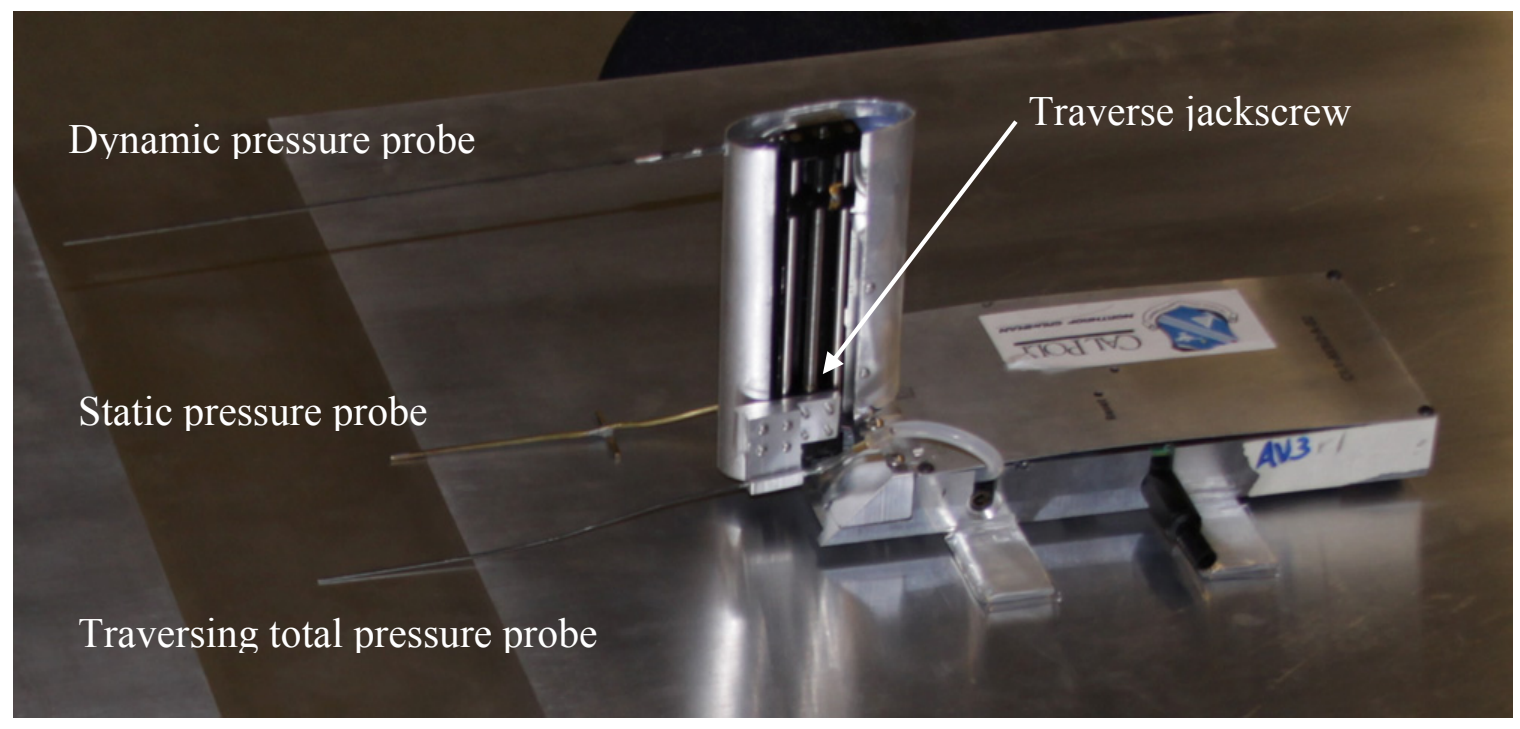

Figure 51. Boundary Layer Data System with probe locations shown. 


\section{BLDS Data Reduction}

For the test, the BLDS was programmed to make 0.010 inch steps from 0.000 inches to 0.22 inches in order to generate a velocity profile for the CCR to validate against. It is assumed that the velocity is at the probe center. With a 0.032 inch outer diameter probe, the data ranges from 0.016 inches to 0.236 inches. Data was gathered at freestream velocities of $21.9 \mathrm{fps}, 51.4 \mathrm{fps}, 81.1 \mathrm{fps}, 110.7 \mathrm{fps}, 140.3 \mathrm{fps}$, and $170.0 \mathrm{fps}$. The

maximum freestream velocity was increased due to the flat plate blockage. Issues arose with noise in the lowest freestream velocity, so this data was ignored. Velocities at or above 81.1 fps had Reynolds numbers greater than 500,000, suggesting transition may be occurring which is consistent with the data. By applying a curve fit to the BLDS data, the velocity at the CCR data location was determined to be $146.3 \mathrm{fps}$ for the $170.0 \mathrm{fps}$ freestream velocity.

\section{CCR Flat Plate Data Reduction}

Data was acquired twice at each velocity at $250,000 \mathrm{~Hz}$ for one second. An example of the raw data is shown in Figure 52. To yield proper correlations, the data was normalized by the mean of the respective channel, centering the data about zero. Once normalized, Channel 7 was inverted. As seen in Figure 52, Channel 0 has fluctuations in the positive direction while Channel 7 is in the negative direction. Figure 53 shows the normalized dataset. The cross correlation completed with MATLAB is shown in Figure 54. This correlation yields a velocity of $0.25 \mathrm{fps}$ which is much lower than the anticipated $146.3 \mathrm{fps}$. The correlation shows a distinct pattern which suggests the noise is the main source of the actual correlation. Attempts to remove this noise from the dataset are discussed below. 


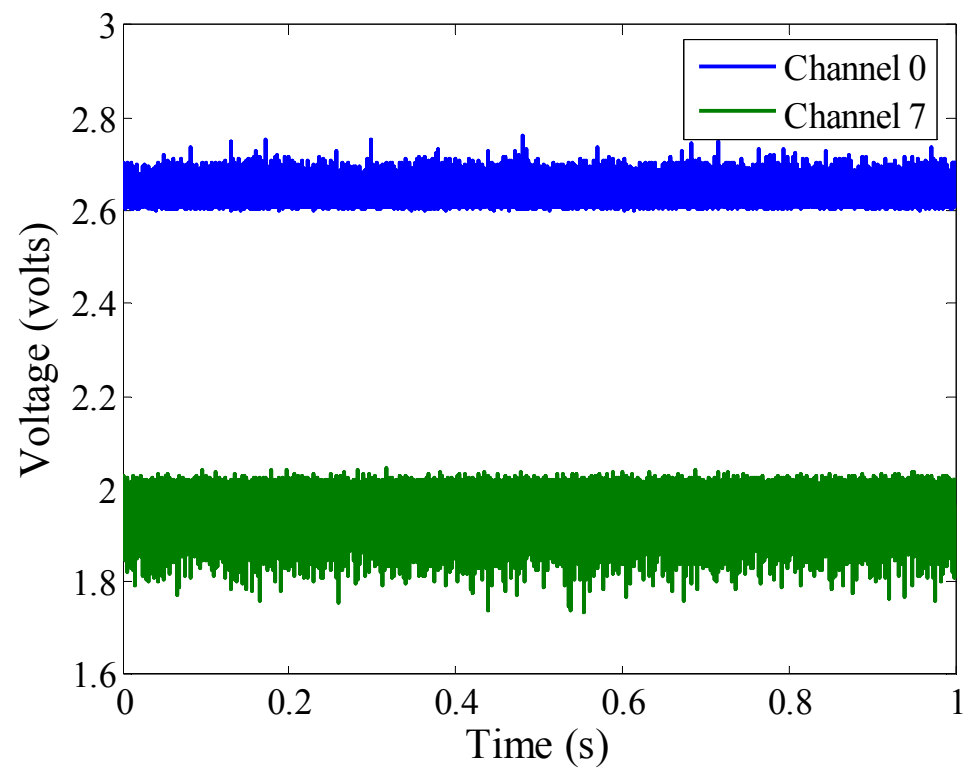

Figure 52. Second dataset from 170.0 fps run taken at $250 \mathrm{kHz}$ sampling rate.

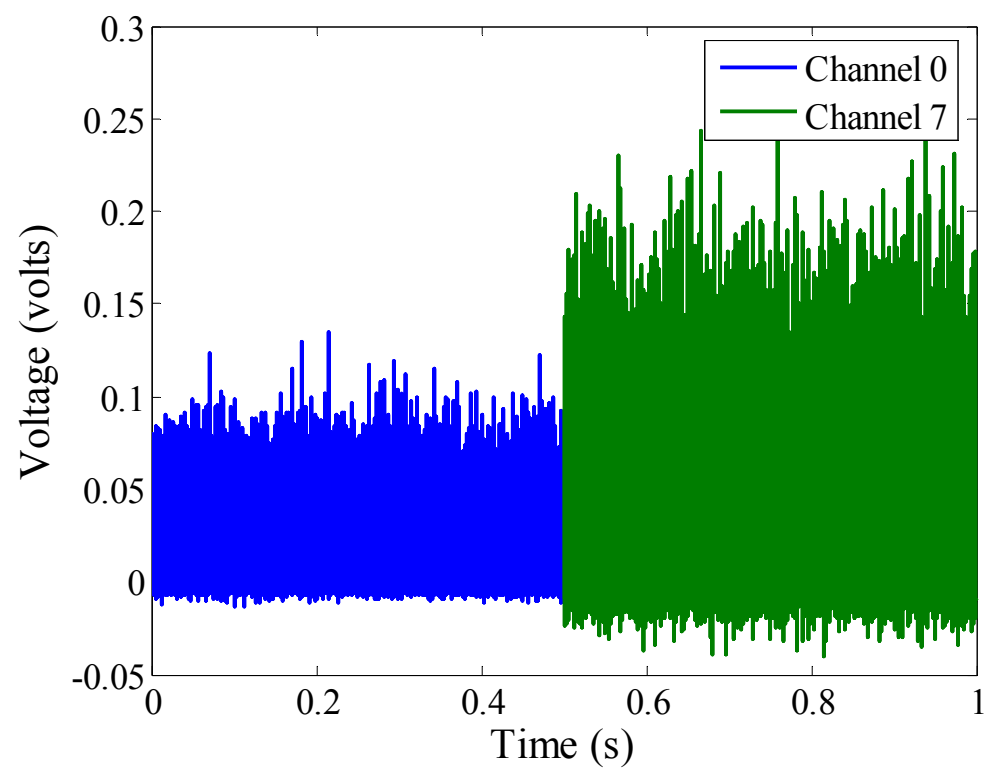

Figure 53. Normalization of second dataset from $170.0 \mathrm{fps}$ run taken at $250 \mathrm{kHz}$ sampling rate. Half of each dataset is shown for clarity. 


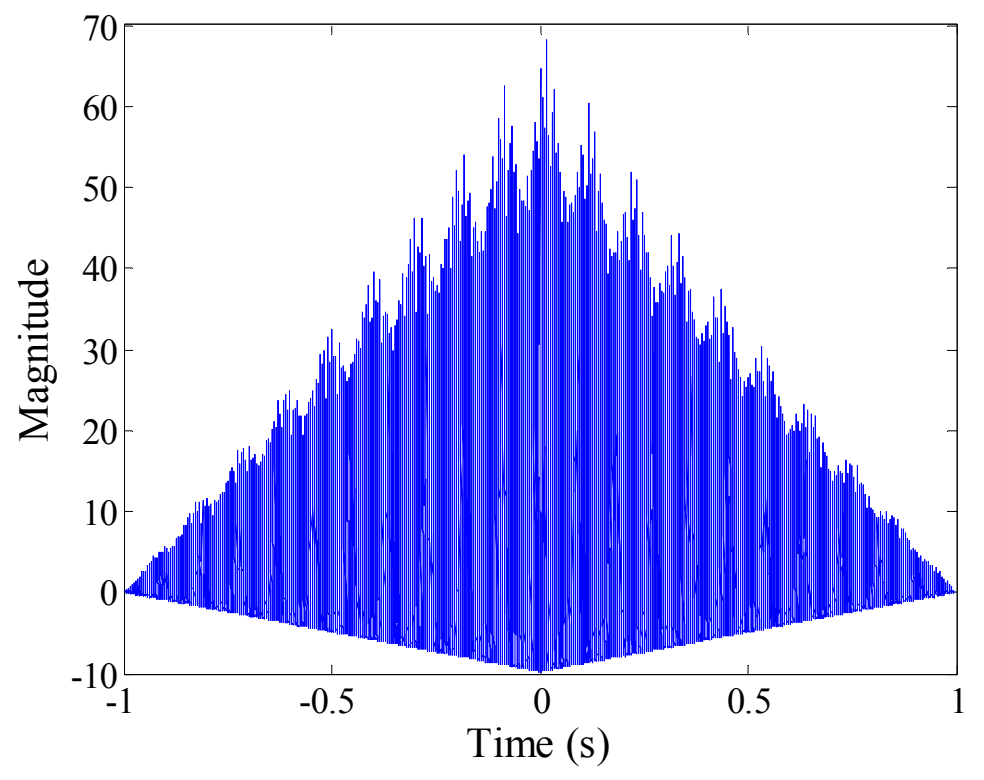

Figure 54. Cross correlation of normalized second dataset from 170.0 fps run.

\section{Software Filtering}

Various digital filtering was applied in an attempt to remove noise in the measurements and improve correlation. A high pass filter was first applied to the data. For the 170.0 fps dataset, a minimum frequency of approximately $70 \mathrm{kHz}$ should yield sufficient data. A $25 \mathrm{kHz}$ cutoff frequency was chosen, in theory removing the $8 \mathrm{kHz}$ noise while not affecting the higher frequencies of interest. This yielded a correlation similar to Figure 54. Since the high pass data was still noisy but possibly useful, a low pass filter was applied with the purpose of smoothing the high pass filtered data. A $10 \mathrm{kHz}$ cutoff frequency was utilized. Overall, this yielded smoothed data, however there was no improvement in the cross correlation. Lastly, a low pass filter was applied to the raw data with no change in the cross correlation. These filters were applied with MATLAB. Filtering features in LabVIEW were also investigated, but MATLAB offered greater control over the filter variables. 
While investigating the data, it was theorized that smoothing could possibly yield an improved correlation. A moving time average was first applied. The desired number of points to average is specified. The overall number of points averaged is removed from the number of points in the dataset. For instance, if 1,000 points were measured and a 10 point moving time average applied, then 990 points would remain in the smoothed dataset. This is one method to compensate for averaging the endpoints. This methodology was shown to yield improved results illustrated in Figure 55. The moving time average used 25,000 points for this particular data. The large number of points required suggests the data is extremely noisy.

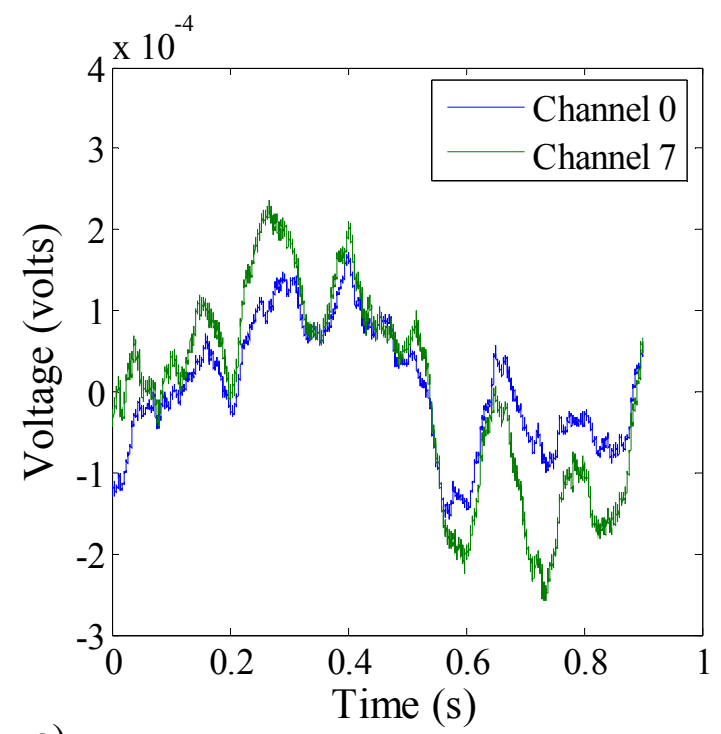

a)

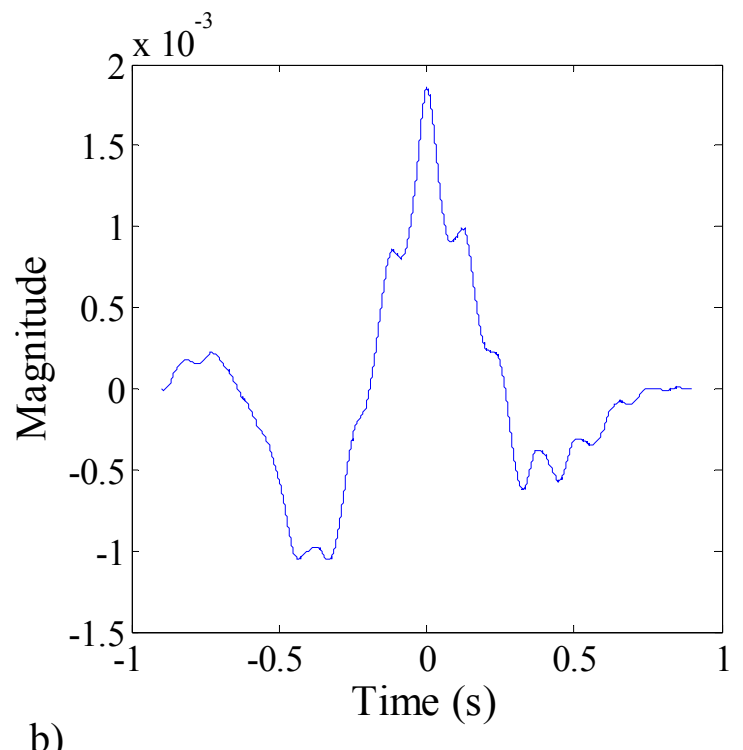

b)

Figure 55. Wind tunnel run at $\mathbf{1 7 0 . 0}$ fps illustrating a) the moving time averaged data and b) the cross correlation of Channel 0 and Channel 7.

Despite the visually improved data, the cross correlation still had a time delay of zero seconds. It was thought that unsmoothed noise in the measurements was continuing to cause a sharp correlation at zero seconds. Upon further inspection of the data, it was determined there was more noise than previously thought. Figure 56a shows $180 \mathrm{~Hz}$ oscillations while a smaller $x$ scale in Figure $56 \mathrm{~b}$ shows $7800 \mathrm{~Hz}$ and $8161 \mathrm{~Hz}$. Utilizing 
MATLAB's Discrete Fourier transform, all the frequencies within the data can be investigated. The leftmost peak in Figure 57 represents the $7800 \mathrm{~Hz}$ and $8161 \mathrm{~Hz}$ noise, with the $180 \mathrm{~Hz}$ not visible. Through Figure 57, it becomes apparent that there are additional frequencies which are contributing to the noise. Serious concern with this noise is from close, strong frequencies such as $7800 \mathrm{~Hz}$ and $8161 \mathrm{~Hz}$ which have a distinct time delay. After time averaging the data, a nonphysical time delay could present itself.
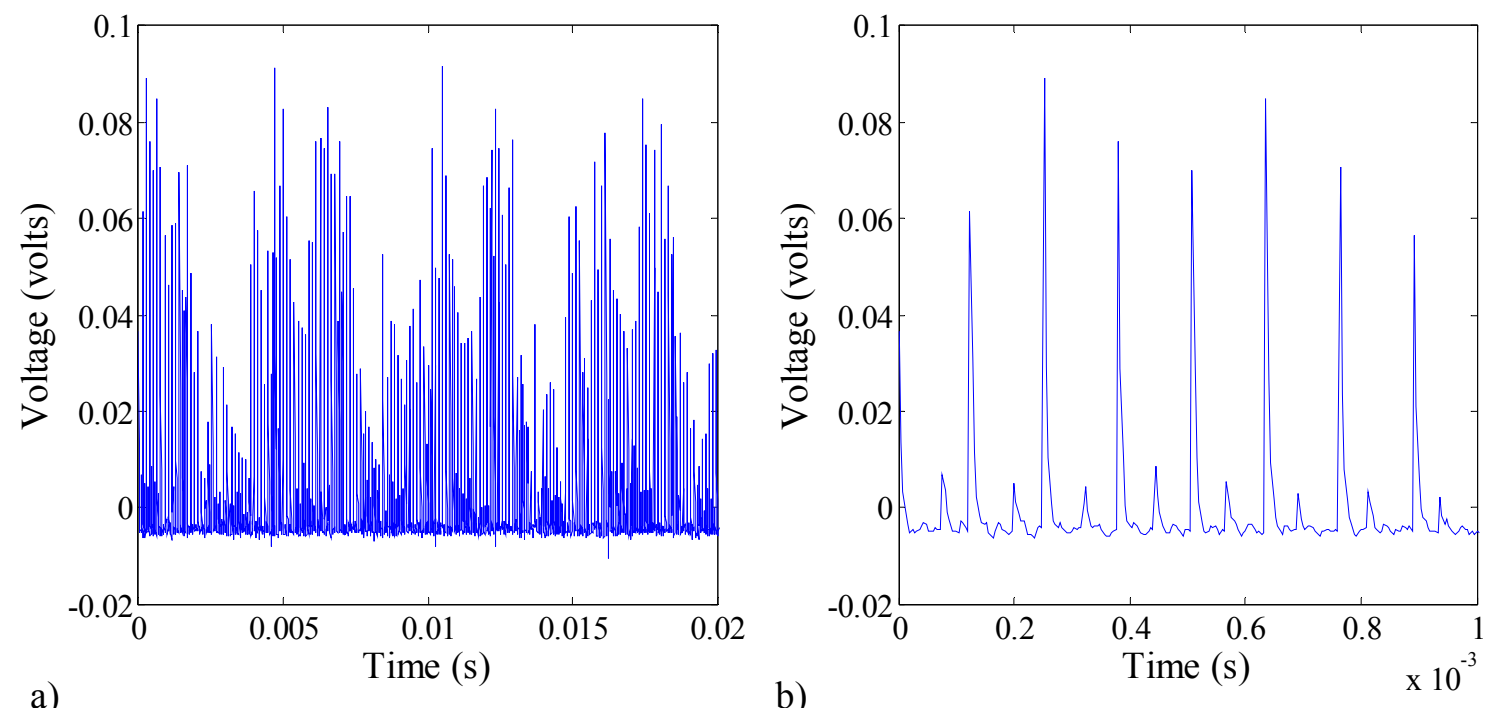

Figure 56. Data from Channel 0 of $\mathbf{1 7 0 . 0}$ fps run with $x$-axis scaled to a) 0.02 seconds and $b$ ) 0.001 seconds.

Upon discussion with Russell Westphal, the variable frequency drive (VFD) of the two foot by two foot wind tunnel was suggested as a noise source. To test this, the full data acquisition system was placed near the tunnel. Data was taken with the tunnel on and off. Figure 58 shows the clear difference in noise due to the VFD. It was determined that while possible to shield the data system, the CCR itself would require shielding, as it acts as an antenna. Since the CCR could not be shielded, a new approach was required to validate the CCR. 


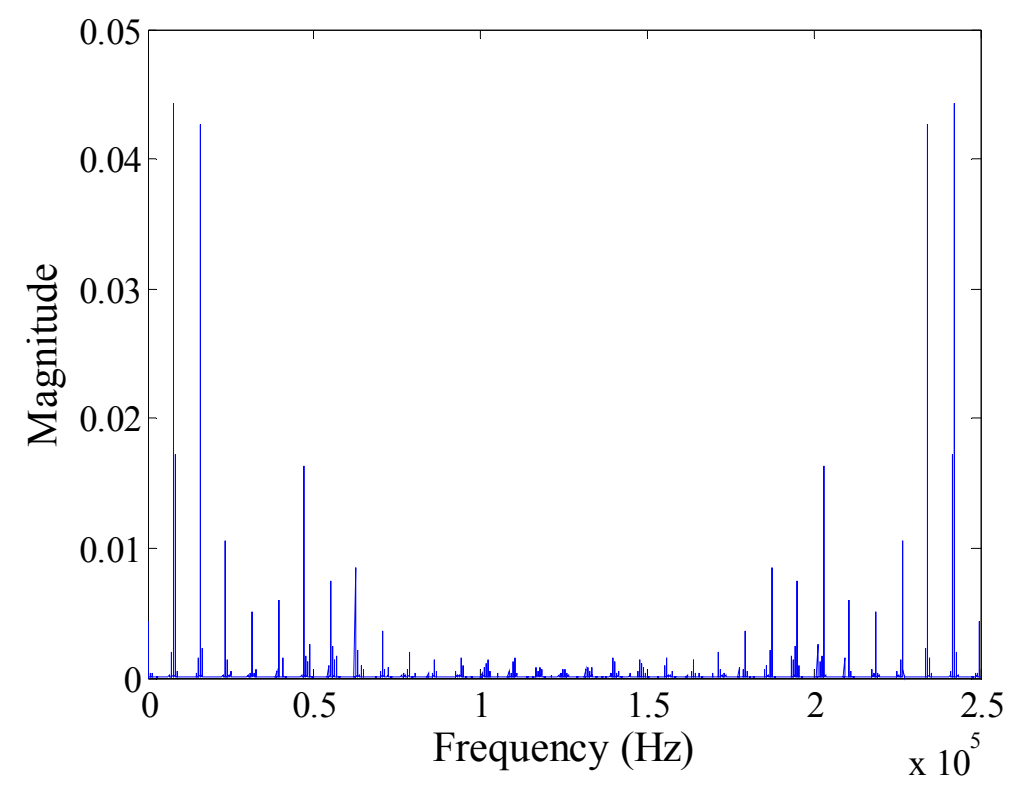

Figure 57. Discrete Fourier transform of Channel 0 from 170.0 fps run.

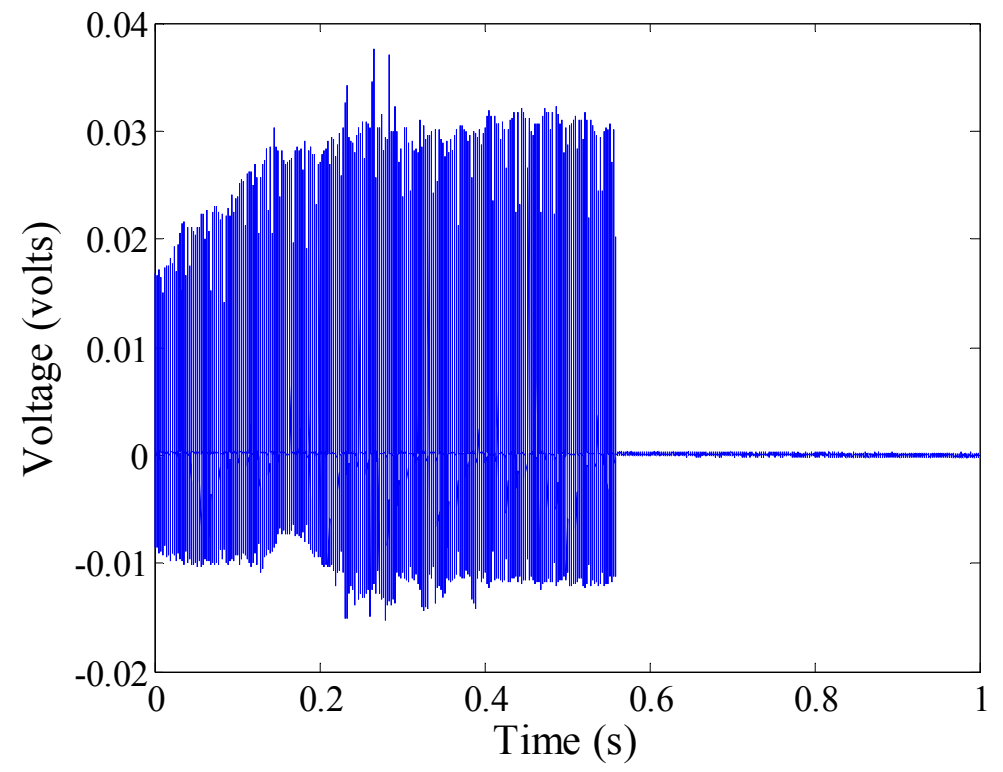

Figure 58. Data taken while the variable frequency drive is on (noise on left) and off (clean data on right). 


\section{Subsonic Flow Bench Validation}

Once it was determined the two foot by two foot tunnel would be unacceptable, a new validation technique needed to be determined. Most large wind tunnels have VFDs to control the freestream velocity. However, flow benches often do not and would therefore be an option. A drawback to a flow bench is poor flow characteristics and limited instrumentation options. Due to the drawbacks, it was decided to only validate the functionality of the CCR to the extent that it can differentiate between two differing flow velocities.

\section{Flow Bench Apparatus}

The Cal Poly Mechanical Engineering modular open jet flow bench manufactured by TecQuipment was decided to be utilized for the validation. The motor only has only one power setting and is not equipped with a VFD. To make crude velocity variations, the flow bench has a butterfly valve. It was undesirable to make a flat plate for the experiment since it would require a CNC mill to machine a slot for the CCR base. To avoid this, the insert designed for the two foot flat plate was placed flush against one of the walls on the flow bench. This allows a developed boundary layer to flow over the CCR while making measurements. The insert was held in place with a large vice, pictured in Figure 59. It is difficult in the image to distinguish where the flow bench wall intersects the flat plate insert because the wall is clear. Katpon ${ }^{\circledR}$ tape was applied between the flat plate insert and the flow bench wall to ensure a better transition between the surfaces. The CCR is not visible in Figure 59. Figure 60 shows the installed CCR and Kapton ${ }^{\circledR}$ tape. 


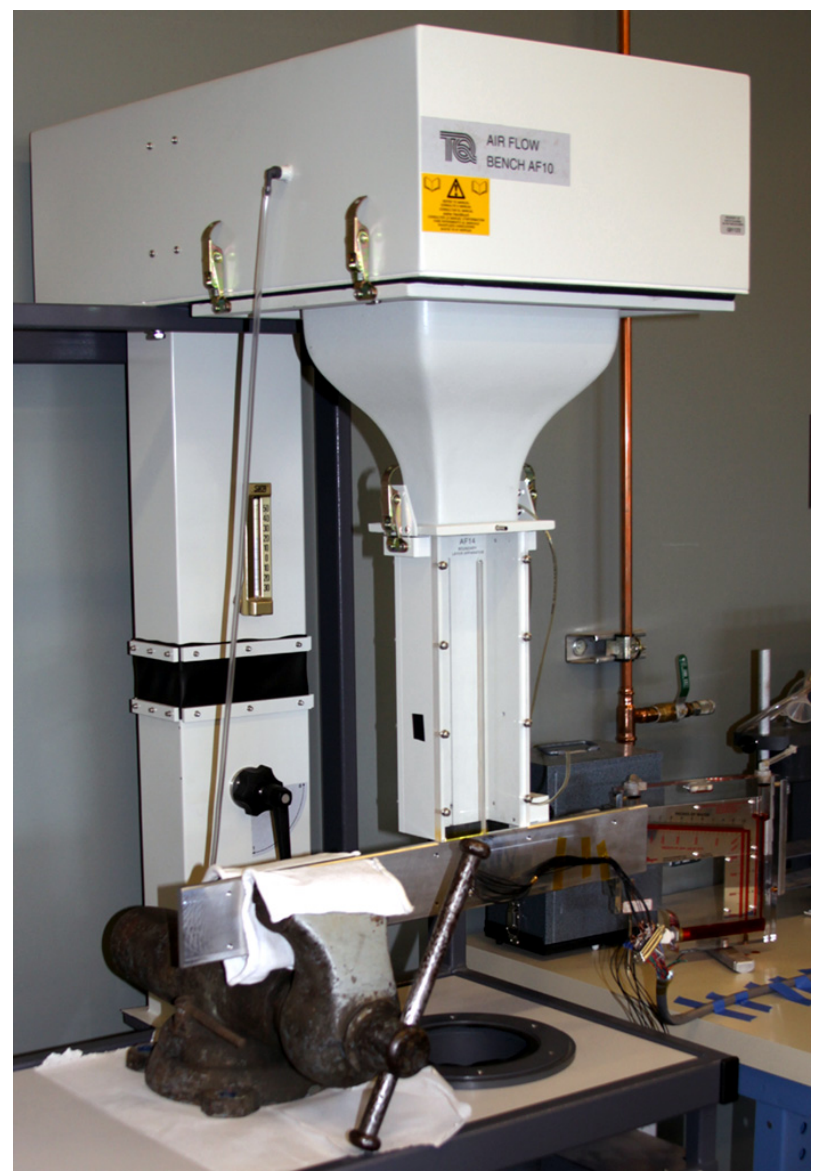

Figure 59. Image of flow bench with flat plate insert held up against test section wall.

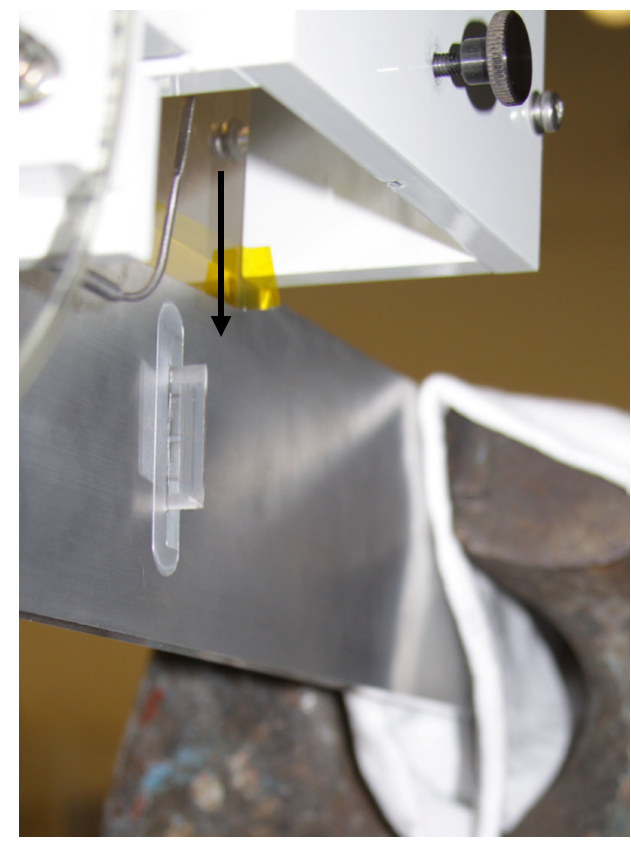

Figure 60. CCR installed on flat plate insert with vice in background. The arrow indicates flow direction. 


\section{CCR Flow Bench Data Reduction}

The data from the flow bench proved to be free of noise from a VFD, as expected. Additional post processing techniques were applied to the data beyond what was described in CCR Flat Plate Data Reduction. Passive analog low pass filtering was applied to two datasets as well. A cutoff frequency of $75 \mathrm{kHz}$ was built into the filter. The passive analog low pass filtering did not yield a correlation.

One dataset of interest had data with differing downward slope. By subtracting a linear curve fit from the data, the slope could be removed, shown in Figure 61. The sloped data would only yield zero correlations. By correcting for the differing slopes, cross correlation time delays resulted. An example of the slope corrected, moving time averaged data yielding a velocity of $94.7 \mathrm{fps}$ is shown in Figure 62.

Since the moving time average technique yielded a correlation, a study on the variation of the number of averaged points was completed. Figure 63 shows that when the number of averaged points increases, the velocity decreases. This study illustrates the problematic use of the moving time average to yield a time delay. Because of this, additional smoothing techniques were investigated. The half velocity dataset is also shown in Figure 63. Unexpectedly, if the same number of time averaged points is taken, the half velocity dataset yields higher velocities than the full velocity dataset. This shows that as more smoothing is applied, the data goes from having zero to infinite correlation, for an infinitely large dataset. 


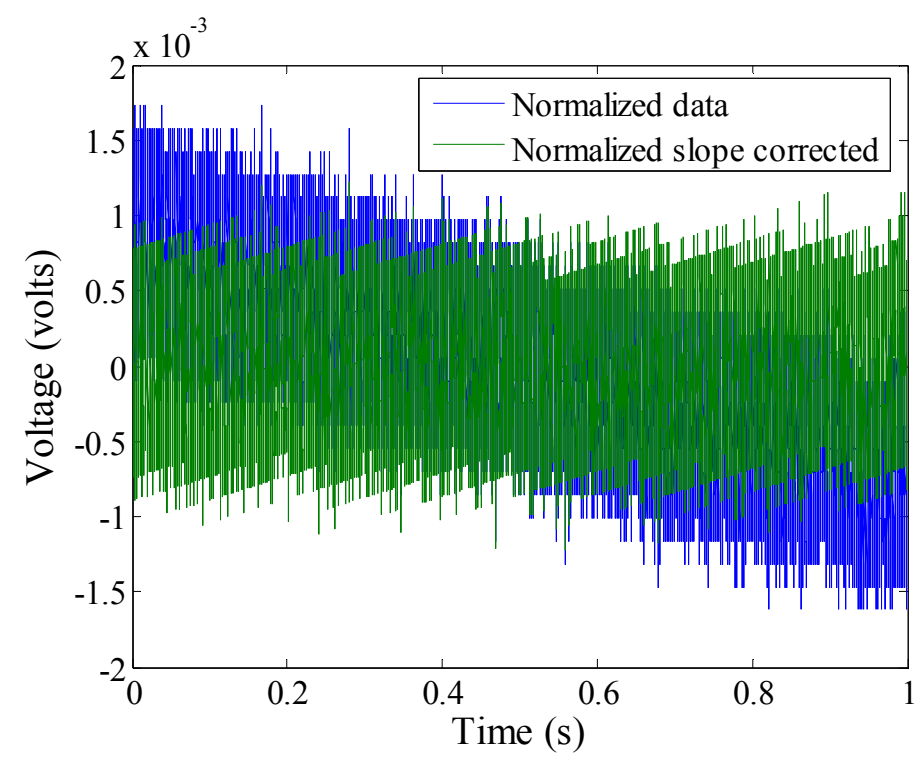

Figure 61. Slope correction in data from flow bench test.

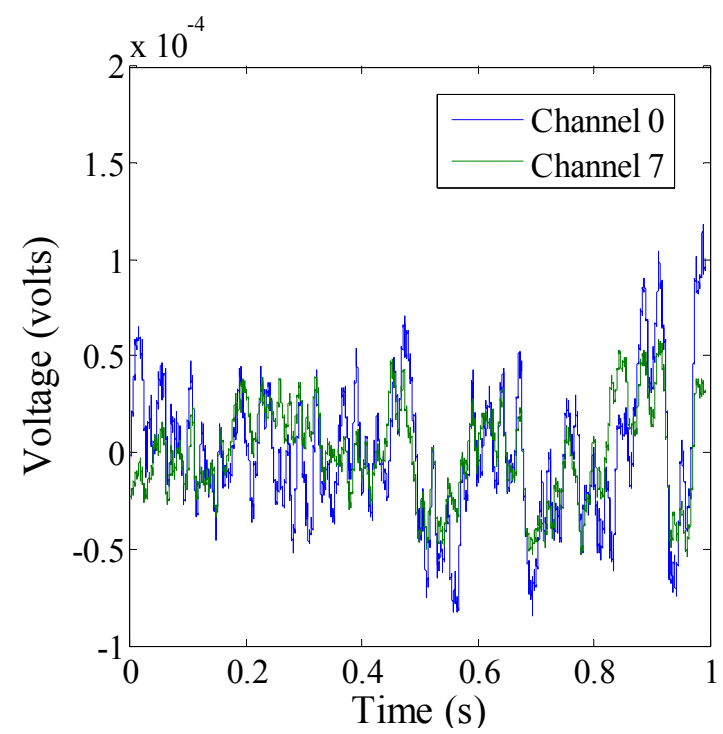

a)

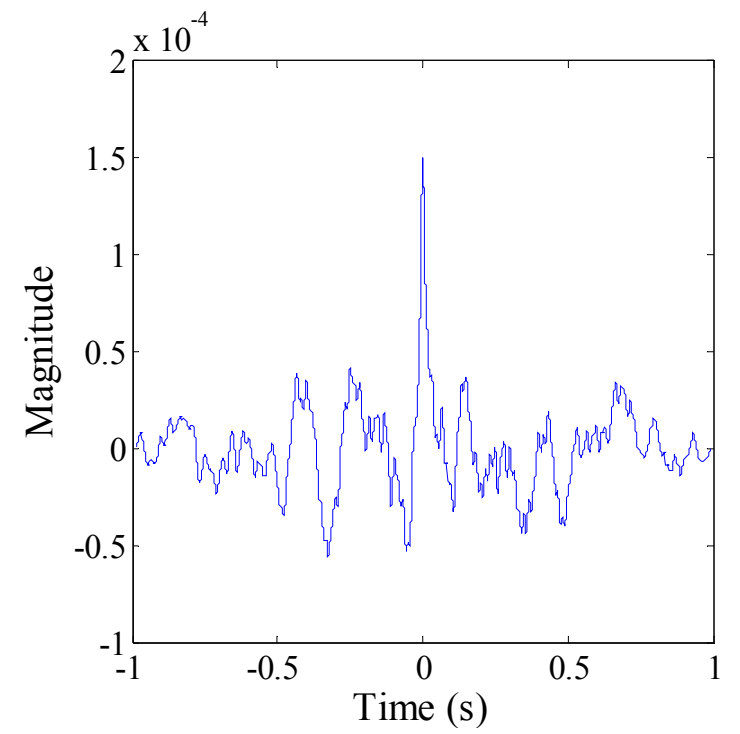

b)

Figure 62. The raw data shown in a) is slope corrected, normalized, and 2,000 point moving time averaged. The correlation in b) shows the velocity of $94.7 \mathrm{fps}$. 


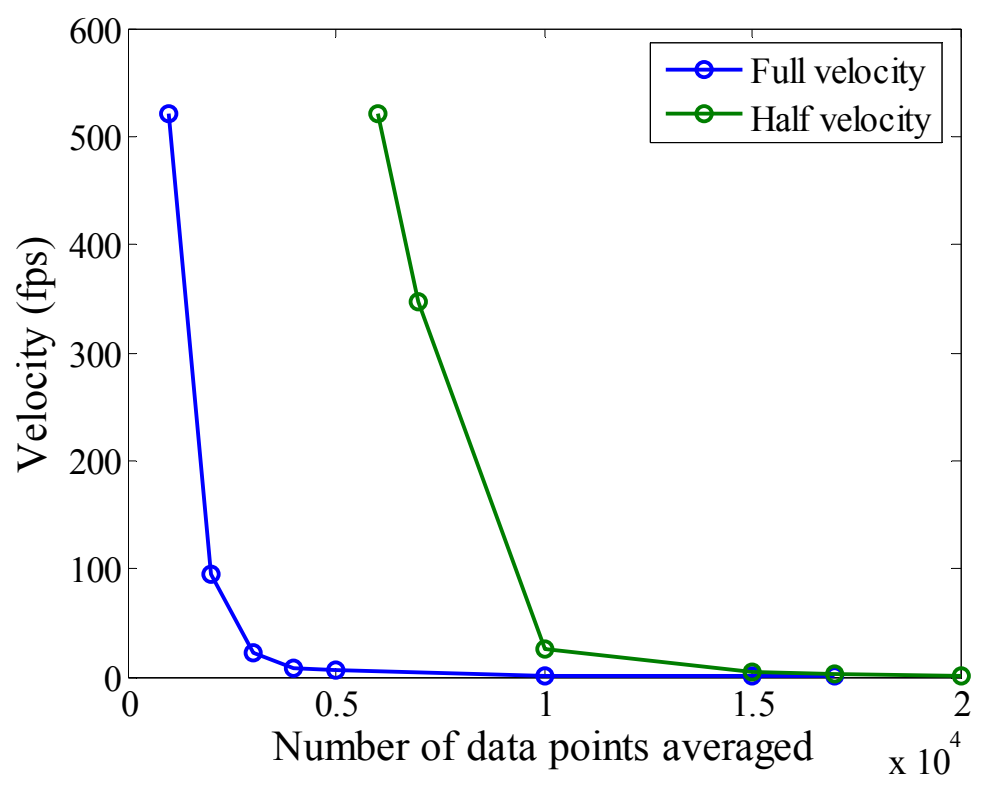

Figure 63. Velocity variation as the number of moving time averaged points increases.

MATLAB's smoothing function implements variations upon the moving time average. A weighted linear least squares $2^{\text {nd }}$ degree polynomial model which assigns lower weights to outliers was implemented on the data. Because of the increased data manipulation, the time to evaluate data was greatly increased. To decrease the time, $20,000,50,000$, and 70,000 of the 250,000 data points were analyzed. The number of points averaged was also varied from 4 to 50 . All results yielded a correlation of zero seconds. The same smoothing technique was applied without applying lower weights to the outliers. Again, all combinations yielded correlations of zero seconds.

\section{Subsonic Validation Summary}

The subsonic validation of the CCR was unsuccessful. First, issues arose with the robustness of the CCR substrate. The final testable CCR only allowed one point to be validated. This would be sufficient for an initial validation, but it is important to characterize further issues such as flow angularity and flow reversal. The extreme near wall measurement locations could yield incorrect data and also need to be validated. 
Since the voltage taps were damaged, these also cannot be tested. Second, due to the VFD in the Mechanical Engineering wind tunnel, there are unacceptably high noise levels which cannot be shielded. A flow bench was therefore utilized to make measurements. The flow bench is poorly instrumented and no velocity measurements are available. However, the velocity can be varied with a butterfly valve. Data was taken at two velocities. Ideally, the cross correlation will yield a slower velocity reading for the slower freestream velocity. Since no velocity measurements are being made, only the rake functionality is tested, not the accuracy or reliability of the data. Third, was found that some sort of filtering would be required to yield data. Low pass, high pass, and band pass filtering was applied, all with no success. A weighted linear least squares $2^{\text {nd }}$ degree polynomial moving time average model which assigns lower weights to outliers was implemented on the data. All the data points had a zero correlation. A moving time average was implemented with success. However, it was found that as the number of points averaged increases, the time delay in the data also increases. One option would be to calibrate the CCR readings to a known velocity in future tests to determine the number of points to average. Lastly, comparing the low and high velocity runs showed that if the same number of points is averaged, the low velocity run will yield a higher velocity than the high velocity run. For these reasons, the validation of the CCR to date is considered unsuccessful.

\section{Future Suggestions}

Care needs to be taken in the handling of the CCR. One thought is to place a nonconductive epoxy over the soldered patches to better keep the wires in place. New substrates are currently being manufactured with a $\mathrm{CNC}$ mill, hopefully reducing 
cracking which occurred during the original manufacturing technique. If free of cracks, the CCR substrate will be more robust in its application.

An improved wind tunnel or flow bench without a VFD should be utilized. A flat plate could be machined and implemented on the current flow bench. However, the flow overall has somewhat low quality. Velocity measurement would need to be made in at least one location to allow for validation of the rake.

Lastly, a variable gain differential amplifier should be purchased. Analog Devices manufactures numerous which would function well in this application. The variable gain would allow the signals going into the data acquisition cards to be matched better, yielding the best possible accuracy from the data card. A purchased differential amplifier would also have higher quality internal components than the components utilized to build the differential amplifiers. 


\section{Chapter IV: Application to AMELIA}

\section{FISF Application to AMELIA}

The FISF application to AMELIA poses serious challenges due to temperature gradients, skin friction and pressure gradients, model access, model lighting, and tunnel transients. The 2 foot wing section oil temperature was simply assumed to be the ambient room temperature (model temperature); a good assumption for subsonic flow. However, AMELIA will have high pressure air flowing through the wing structure, rapidly expanding and changing temperature. This will cause large temperature gradients on the model surface. Since the oil is assumed to be the same temperature as the model, the oil temperature will also have large gradients. As discussed previously, roughly $1{ }^{\circ} \mathrm{F}$ corresponds to $1 \%$ uncertainty in a skin friction measurement. To more accurately know the oil temperature, 12 thermocouples will be placed right under the model surface. A high quality infrared camera will also be utilized to make model surface temperature measurements throughout the run. The thermocouples will serve as a verification and possibly calibration of the infrared camera's data. The temperature gradient information will be applied to the skin friction data during the post processing.

Utilizing different approximate solutions of Squire's equation, the skin friction and pressure gradient effects can be taken into account. CFD can be utilized to determine the shear stress gradients allowing for a preliminary uncertainty approximation. Roughly speaking, the leading edge and flap surfaces have the largest gradients, so this should be taken into consideration in validation efforts. 
Due to the NFAC's size, model access is extremely limited. To yield skin friction data on the suction side of the wing, images must be taken on the suction side. To decrease the time to start imaging AMELIA after tunnel shutdown, AMELIA will be inverted. This allows for the suction side of the wing to be viewed from the tunnel floor. Ladders can be used to gain optical access rather than a man lift, requiring less time.

Since the tunnel surface is porous and too large to light, AMELIA will be lit with a diffuse reflector, similar to the one rendered in Figure 64. Two long fluorescent bulbs will be placed spanwise along the leading and trailing edges, providing sufficient lighting for the model. This reflector system will be tested before the wind tunnel test to ensure proper functionality.

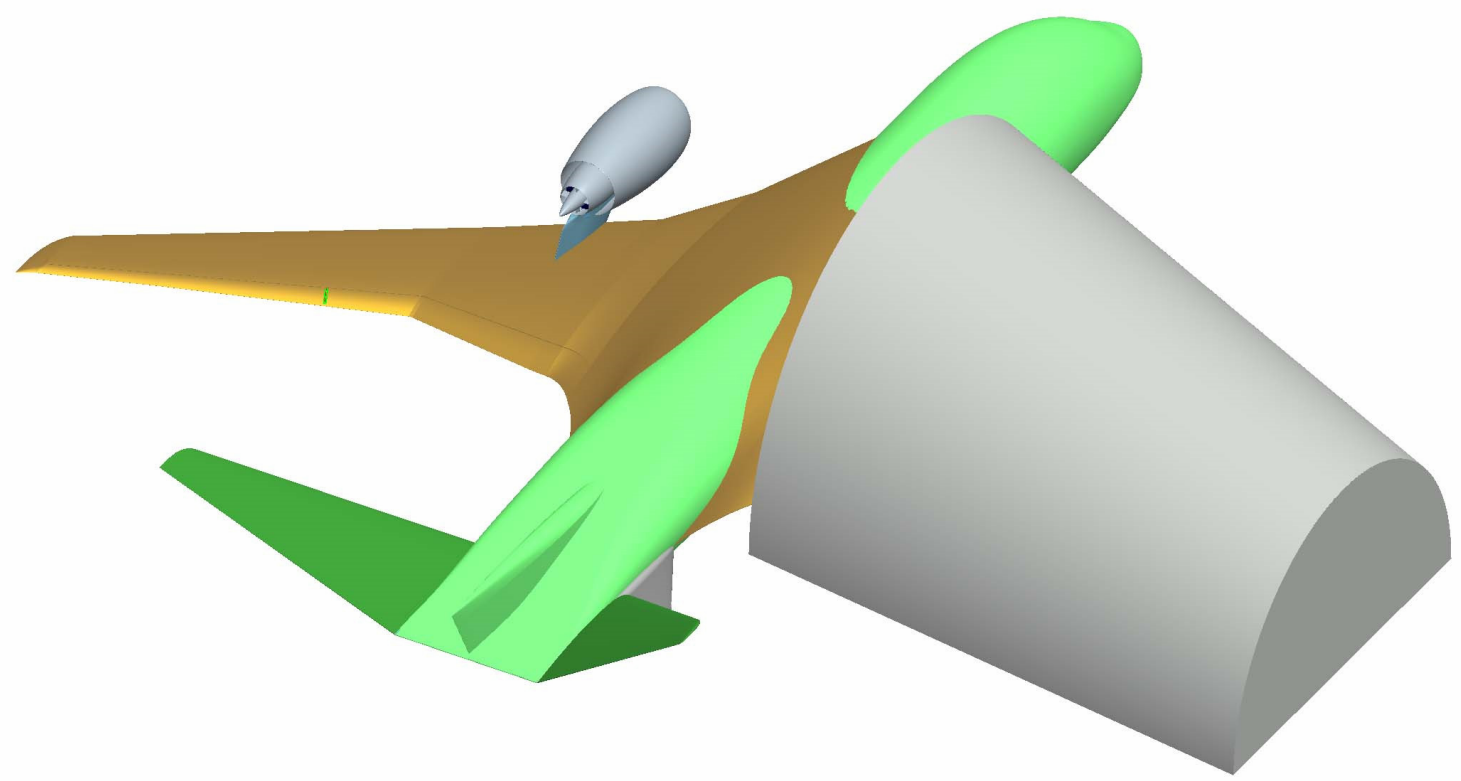

Figure 64. Rendering of a possible diffuse reflector for AMELIA. Lighting would be placed below the wing pointing upward to illuminate the reflector surface.

Lastly, tunnel transients will be dealt with using the procedure outlined in Test Procedure. Some issues may occur with this implementation however. First, the sting is capable of $-5^{\circ}$ to $25^{\circ}$ angle of attack variation, with the positive direction defined above 
the horizon. The blade holding the model was designed with a $-5^{\circ}$ offset, to change the range to $-10^{\circ}$ to $20^{\circ}$ variation. When the model is inverted, the $-5^{\circ}$ blade offset is reversed. This nullifies the $-5^{\circ}$ sting offset, which now varies from $0^{\circ}$ to $30^{\circ}$. Since the model is inverted, the model will see air from $0^{\circ}$ to $-30^{\circ}$, highly undesirable angles of attack to test at. Also, this removes the ability to separate the suction side of the airfoil. Second, the sting itself rotates at about $5^{\circ}$ per minute. This slow movement will make determining the wind on condition for the model extremely difficult. The transients are highly undesirable, making this an important issue.

Since Imron ${ }^{\circledR}$ will most likely be applied, a reevaluation of fiducial options was needed. The painter found that fiducials can easily be masked off and airbrushed into the Imron ${ }^{\circledR}$ once cured. However, the fiducials in this setup have a raised edge which could trip the flow when a clean, non-tripped wing is testing in the NFAC. An etched fiducial which lies below the model surface was also made. This shows promise in that it may not cause the flow to transition. A sample of the painted fiducials is shown in Figure 65 . Testing is required before the final fiducial setup is determined. Lastly, to avoid pressure port blockage, 0.5 psig was applied while the sample was sprayed. The pressure ports were not blocked, but pooling occurred at the paint / port interface. Sanding was found to remove the small pooling which occurred at the interface.

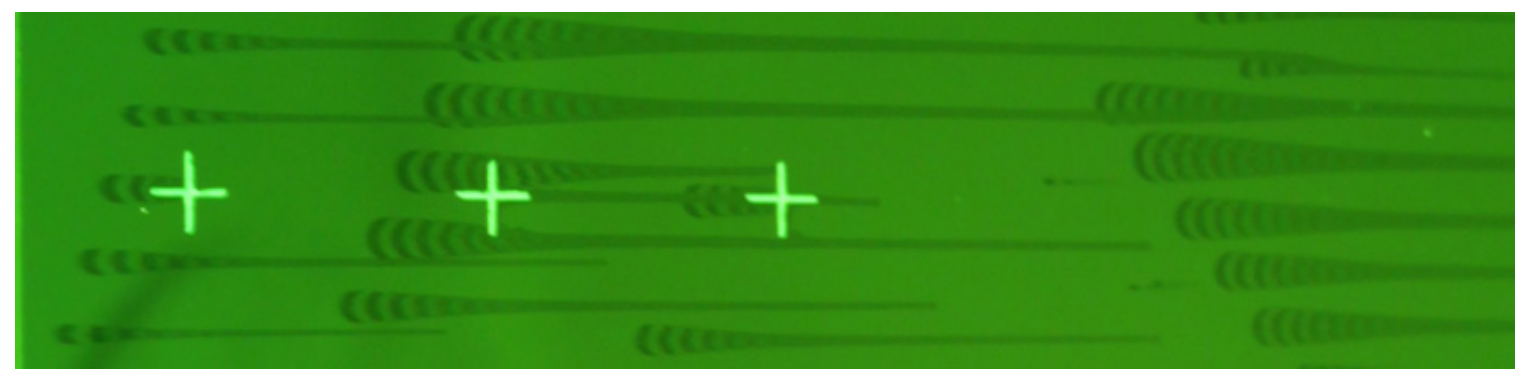

Figure 65. Image illustrating painted fiducials crosshairs and fringes on Imron surface. 
Using the additional uncertainty terms, the complete uncertainty of AMELIA can be estimated. The summary of the uncertainty terms is listed in Table 6. In the best case scenario, the total uncertainty of AMELIA is estimated to be $\pm 4.9 \%$ of $c_{f}$ with a $\pm 1.4^{\circ}$ variation in direction. The worst case scenario occurs at locations with 3D oil flow (wingtip) and high shear shear gradients (leading and trailing edges near slots). At worst, the uncertainty is estimated to be $\pm 11.3 \%$ of $c_{f}$ with a $\pm 2.0^{\circ}$ variation in direction.

Table 6. Summary of AMELIA uncertainty terms.

\begin{tabular}{|c|c|c|}
\hline Error source & AMELIA low uncertainty & AMELIA high uncertainty \\
\hline Oil initial condition & $-0.03 \%$ of $c_{f}$ & $-0.03 \%$ of $c_{f}$ \\
\hline Oil streamline curvature errors & $0 \%$ of $c_{f}$ & $2 \%$ of $c_{f}$ \\
\hline Oil viscosity & $\pm 2.5 \%$ of $v_{o}$ & $\pm 2.5 \%$ of $v_{o}$ \\
\hline Temperature & $\pm 1^{\circ} \mathrm{F}$ of $T_{o}$ & $\pm 1 \%$ of $T_{o}$ \\
\hline Light source wavelength & very small & very small \\
\hline Oil index of refraction & $\pm 0.015 \%$ of $n_{o}$ & $\pm 0.015 \%$ of $n_{o}$ \\
\hline Freestream dynamic pressure & $\pm 0.5 \%$ of $q_{\infty}$ & $\pm 0.5 \%$ of $q_{\infty}$ \\
\hline$d P / d s$ & $0 \%$ of $c_{f}$ & $\pm 0.14 \%$ of $c_{f}$ \\
\hline$d \tau_{w} / d s$ & $0 \%$ of $c_{f}$ & $\pm 10 \%$ of $c_{f}$ \\
\hline Regression and imaging errors & $\pm 2.5 \%$ of $\Delta s$ & $\pm 2.5 \%$ of $\Delta s$ \\
\hline Lens distortion errors & $0 \%$ of $\Delta s$ & $\pm 0 \%$ of $\Delta s$ \\
\hline Startup and shutdown $c_{f}$ errors & $0 \%$ of $c_{f}$ & $1.5 \%$ of $c_{f}$ \\
\hline Human repeatability & $1.0 \%$ of $\Delta s$ & $2.3 \%$ of $\Delta s$ \\
\hline Photogrammetry & very small & very small \\
\hline Experimental repeatability & very small & very small \\
\hline Camera focus & very small & very small \\
\hline Filter variation & very small & very small \\
\hline
\end{tabular}




\section{CCR Application to AMELIA}

Application of the CCR to the AMELIA test will yield valuable data for CFD validation. Velocity measurements of both the jet boundary layer and jet shear layer will be acquired. The CCR span location was chosen to be far from the engine, wingtip, or chord variation effects. An outboard location of 36.6 inches from the centerline was chosen, shown in Figure 66. The rake was placed perpendicular to the trailing edge slot to ensure the substrate incurs no flow angularity. The rake is also perpendicular to the flap dihedral. As the flap deflects, the rake position is held constant relative to the flap trailing edge.

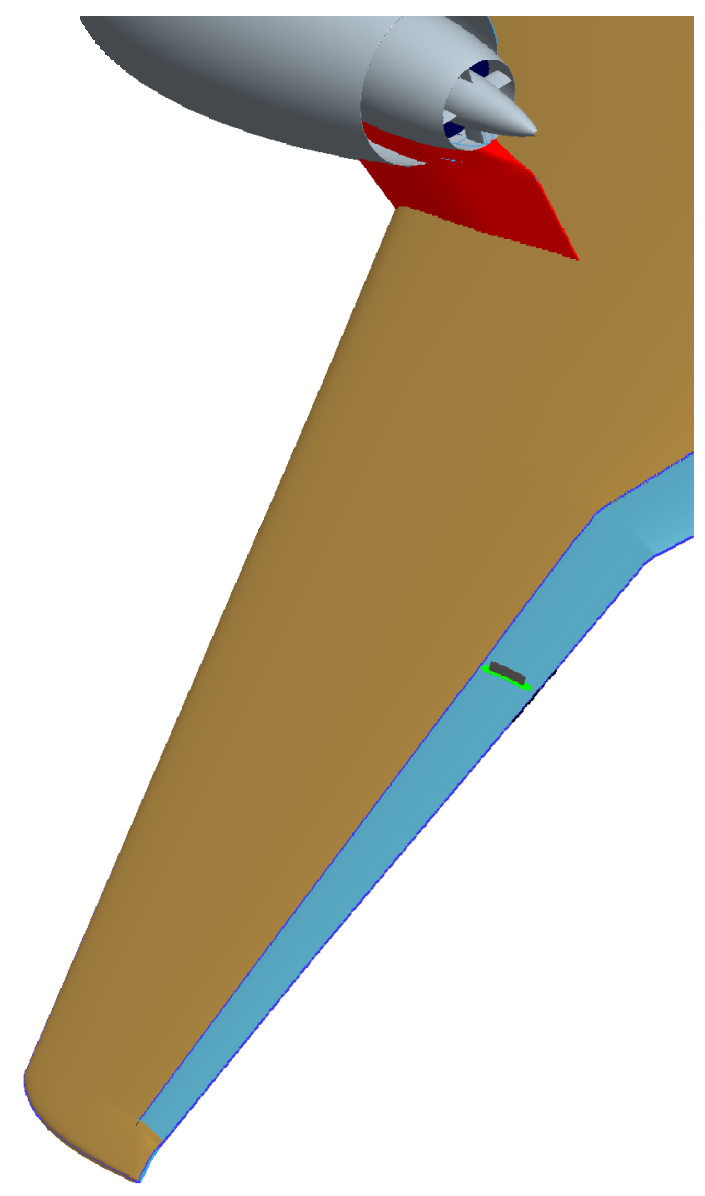

Figure 66. Isometric view of the CCR applied to a rendering of the AMELIA wing. 
There are 20 wires leading from the CCR which lead inboard through a tube, shown in Figure 67. To be able to fit the wires, the jacket over the shielding will need to be removed. These wires lead to an Omnetics micro connector. Larger shielded twisted pair wires then lead into the model body. The data acquisition system will either be placed in the sting fairing behind the model or outside the tunnel in the balance room.

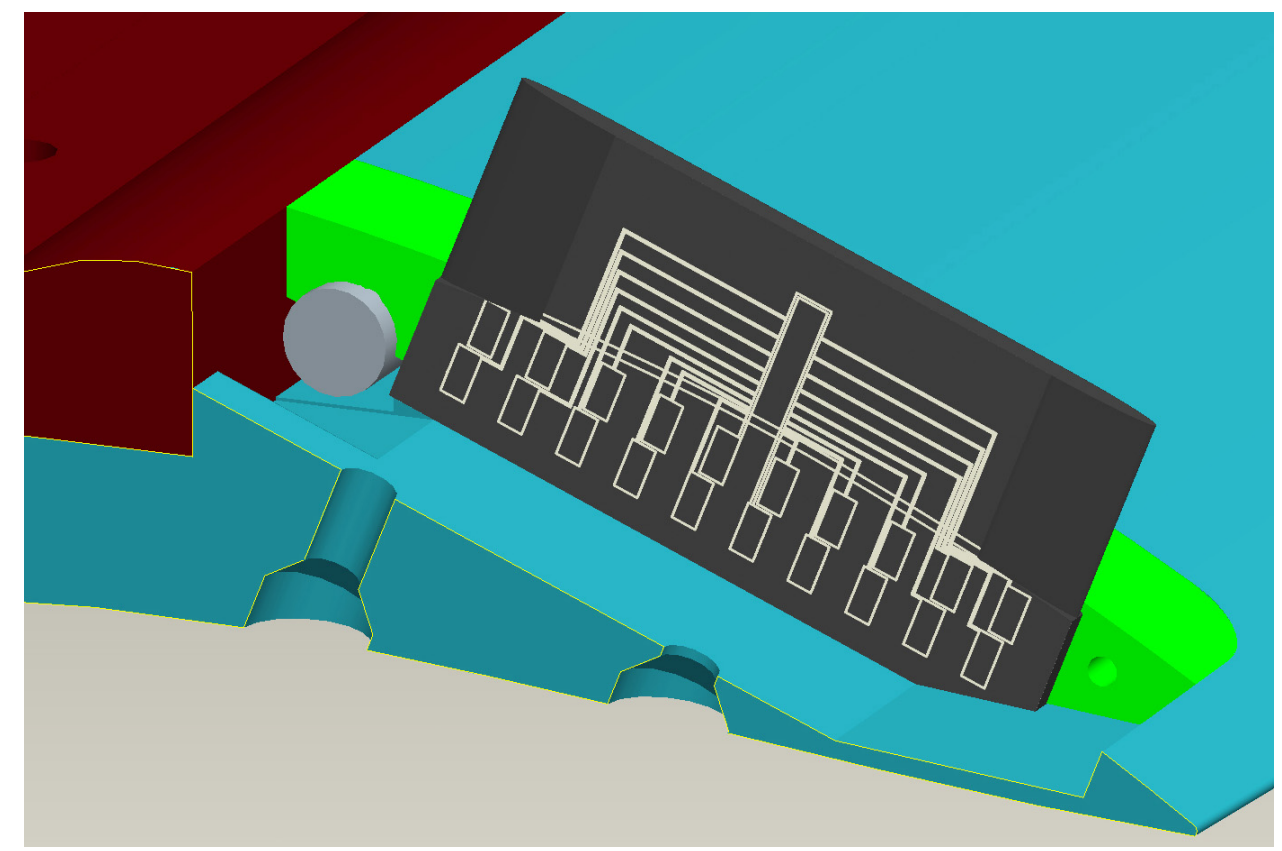

Figure 67. Isometric view of CCR substrate (dark grey), half of CCR base (green), flap (blue) and bottom of wing plenum (red). The platinum mask is shown overlaid on the rake. The light grey circular tube carries the CCR wiring inboard. Two white curved lines represent the flap upper surface relative to the platinum mask.

AMELIA was designed with four flap settings, each having an offblock allowing for CCR installation. There is one universal base for the CCR which fits into the offblock locations in each flap. The Omnetics micro connector allows for simple and rapid removal of the CCR wiring.

Data will be acquired from the CCR at only 10 test points. During these 10 test points, global skin friction and acoustic measurements will be made on AMELIA. Despite the CCR being relatively nonintrusive, it will still cause slot flow blockage and 
additional drag, skewing the force and moment data. At a later time, these data points will be retaken for a comparison of a clean model.

Noise issues from the NFAC's VFD need to be determined. The six motors that drive the tunnel are not near the test section, but it can be assumed that there would be considerable noise from the power systems. Many tests have occurred in the NFAC so noise issues should be well documented with solutions available. 


\section{Chapter V: Conclusion}

The application of the Fringe-Imaging Skin Friction technique to a two foot, nickel plated wing section has been completed. A white diffuse reflector was utilized to ensure proper lighting on the model. Mercury vapor lamps were chosen to light the model. Imaging was completed with a Canon 50D with a bandpass filter applied to the lens to ensure a monochromatic light reaches the camera. The oil was calibrated with a Canon Fenske routine viscometer to $\pm 1.6 \%$ accuracy of $v_{o}$. All of the tests were run at $160 \mathrm{fps}$ for approximately 15 to 20 minutes. To remove the tunnel transients, the model was pitched to $30^{\circ}$ to separate the flow over the wing upper surface.

A continuation of the experimental uncertainty of the FISF technique was completed. The main uncertainties discovered were from human repeatability, photogrammetry, and the use of High Dynamic Range images. Due to the nature of the data acquisition, human repeatability was important to characterize. On average, data within $\pm 1.11 \%$ to $\pm 2.44 \%$ of $\Delta s$ and $\pm 1.27^{\circ}$ to $\pm 2.24^{\circ}$ of $\theta$ can be expected for human repeatability. Photogrammetry was found to be model dependant. For the particular wing blend studied here, the photogrammetry was found to have a negative skew of $1.60 \%$ yielding an uncertainty range of $-6.15 \%$ to $+2.95 \%$ of $\Delta s$. Lastly, HDR images were found to yield particularly poor data if care was not taken. It is advised to avoid post processing of images, as additional fringe noise is added, yielding up to $\pm 25 \%$ uncertainty of $c_{f}$. Various uncertainties were found to be negligible. The experimental repeatability is low, (within the uncertainty of the measurement). If the image is not in focus, the image will be unusable. Within the range of focus usability, the variation between data is less than the 
human repeatability. It is recommended to take images at a variety of focus distances if autofocus fails to operate effectively. Lastly, the filter variation was found to yield a negligible difference in data. The skin friction measurements were found to be in good agreement against known Preston tube data.

In application to the AMELIA test, care must be taken to reduce traditional uncertainty sources as much as possible. Fiducials will be applied by the model maker, yielding low photogrammetry errors. An IR camera will be utilized in conjunction with the surface thermocouples to accurately measure the oil temperature variation during the test. This vastly reduces the uncertainty due to viscosity variations. Tunnel transient reduction in the NFAC needs further investigation before testing occurs. The NFAC has been thoroughly characterized and accurate freestream properties are measured during testing. The $6 \mathrm{~nm}$ filter may be utilized if zoom is needed to correctly image the model. If a full frame image is required however, the $35 \mathrm{~nm}$ filter is an acceptable replacement. Care must be taken to accurately focus the camera. Lastly, the human repeatability uncertainty will be applied to the final dataset. If future improvements are made to the automatic fringe identification software, the data could be processed again to further reduce the uncertainty in the measurement.

A Cross Correlation Rake was designed, fabricated, tested, and validated unsuccessfully at subsonic speeds. The CCR allows high resolution, near wall velocity measurements to be made making the device advantageous over other measurement options. The AMELIA application defined the CCR's design requirements. The quartz substrate size of 1.1 inch by 0.55 inch was determined based on the flap location on AMELIA. The platinum loop spacing was required to yield a sufficient resolution on the 
flap while maintaining a reasonably low data sample rate. Utilizing CFD solutions, the positioning of the desired velocity measurements were chosen. Care was taken to remove noise from the voltage measurements by utilizing batteries on both the platinum loop and on the power supply for the differential amplifiers. Despite this, the data was still found to vary $10 \%$ more than the manufacturer quoted data card accuracy. No improvement on this measurement could be made despite multiple efforts.

The CCR was unsuccessfully validated. The CCR was first tested on a flat plate at subsonic speeds. A Boundary Layer Data System was employed at the same axial position along the flat plate. Correlations in the data occurred due to noise from the variable frequency drive used to control the wind tunnel motor. Low pass, high pass, and band pass filters were applied to the data, both analog and digital, with no improvement. A flow bench was utilized to validate the CCR's ability to discern a high speed flow from a low speed flow. Again, similar analog and digital filtering techniques were applied with no success. A moving time average yielded results that were dependent upon the number of data points averaged. A study showed that if the same number of data points were averaged, the high speed flow would yield a lower correlated velocity than the low speed flow. Therefore, with this post processing technique, the CCR is not capable of distinguishing velocity variations.

For AMELIA, work needs to continue on the validation of the CCR before it will be applied in a full scale test. Issues arose with soldering the wires to the platinum patches which would make installation on AMELIA difficult due to the requirement of a robust measurement device. Care must be taken during installation to ensure that the quartz substrate is held in place firmly while not damaging the quartz. A continued effort to 
validate the CCR will be made. A differential amplifier will be purchased allowing better tuning of the data amplification. Discussions will continue with NASA Glenn to determine an adequate filtering system which would yield correlatable data. 


\section{References}

${ }^{1}$ Marshall, D. D., and Jameson, K. K., "Overview of Recent Circulation Control Modeling Activities at Cal Poly," 48th AIAA Aerospace Sciences Meeting and Exhibit, AIAA 2009-0045, January 2010.

${ }^{2}$ Golden, R. M., and Marshall, D. D., "Design and Performance of Circulation Control Flap Systems," $48^{\text {th }}$ AIAA Aerospace Sciences Meeting and Exhibit, AIAA 2010-1053, January 2010.

${ }^{3}$ Englar, R. J., "Development of the A-6/circulation Control Wing flight Demonstrator Configuration," DTNSRDC, Rept. ASED-79/01, January 1979.

${ }^{4}$ Loth, J. L., Fanucci, J. D., and Roberts, S. C., "Flight Performance of a Circulation Control STOL Aircraft," Journal of Aircraft, vol. 13, no. 3, 1976, pp. 169-173.

${ }^{5}$ Englar, R. J., Gaeta, R. J., Lee, W. J., and Leone, V., "Development of Pneumatic Over-the-Wing Powered-Lift Technology; Part I: Aerodynamic/Propulsive," 27 $7^{\text {th }}$ AIAA Applied Aerodynamic Conference, AIAA 2009-3942, Atlanta, GA, June 2009.

${ }^{6}$ Gaeta, R. J., Englar, R. J, and Avera, M., "Development of Pneumatic Over-the-Wing Powered-Lift Technology; Part II: Aeroacoustics," $27^{\text {th }}$ AIAA Applied Aerodynamic Conference, AIAA 20093941, Atlanta, GA, June 2009.

${ }^{7}$ Blessing, B., "Entraining Engine Flow Using a Circulation Control Wing," Thesis, California Polytechnic State University, San Luis Obispo, September 2010.

${ }^{8}$ Jameson, K. K., Marshall, D. D., Ehrmann, R. S., Paciano, E. N., and Golden, R., "Design and Wind Tunnel Testing of Cal Poly's AMELIA 10 foot Span Hybrid Wing-Body Low Noise CESTOL Aircraft," $27^{\text {th }}$ International Congress of the Aeronautical Sciences, September 2010.

${ }^{9}$ Cagle, C. M. and Jones, G. S., "A Wind Tunnel Model to Explore Unsteady Circulation Control for General Aviation Applications," $22^{\text {nd }}$ AIAA Aerodynamic Measurement Technology an dGround Testing Conference, AIAA 2002-3240, June 2002.

${ }^{10}$ Angle, II, G., O'Hara, B., Huebsch, W., and Smith, J., "Experimental and Computational Investigation into the Use of the Coanda Effect on the Bell A821201 Airfoil," Applications of Circulation Control Technology, edited by R. D. Joslin and G. S. Jones, Vol. 214 of Progress in Astronautics and Aeronautics, chap. 9, American Institute of Aeronautics and Astronautics, Inc., 2006, pp. 277291.

${ }^{11}$ Jones, A., Edstrand, A., Chandran, M., Wetzel, D., Liu, F., and Cattasfesta, L., "An Experimental Investigation of Unsteady and Steady Circulation Control for an Elliptical Airfoil," $48^{\text {th }}$ AIAA Aerospace Sciences Meeting, AIAA 2010-346, January 2010.

${ }^{12}$ Wood, N. J., and Conlon, J. A., "The Performance of a Circulation Control Airfoil at Trnasonic Speeds, “ AIAA Paper 83-0083, January 1983.

${ }^{13}$ Alexander, M. G., Anders, S. G., Johnson, S. K., Florance, J. P., and Keller, D. F., "Trailing Edge Blowing on a Two-Dimensional Six-Percent Thick Elliptical Circulation Control Airfoil Up to Transonic Conditions," NASA/TM-2005-213545, Langley Research Center, Hampton, Virginia, March 2005.

${ }^{14}$ Jones, G. S., Yao, C., and Allan, B. G., "Experimental Investigation of a 2D Supercritical CirculationControl Airfoil Using Particle Image Velocimetry," $3^{\text {rd }}$ AIAA Flow Control Conference, AIAA 2006-3009, June 2006.

${ }^{15}$ Wetzel, D., Griffin, J., Liu, F., and Cattafesta, L., “An Experimental Study of Circulation Control on an Elliptical Airfoil," $29^{\text {th }}$ AIAA Fluid Dynamics Conference, AIAA 2009-4280, June 2009.

${ }^{16}$ Collins, S. W., Westra, B. W., Lin, J. C., Jones, G. S., and Zeune, C. H., "Wind tunnel testing of powered lift, all-wing STOL model," The Aeronautical Jounral, vol. 113, no. 1140, February 2009, pp. 129137.

${ }^{17}$ Lin. J. C., Jones, G. S., Allan, B. G., Westra, B. W., Collins, S. W., and Zeune, C. H., "Flow-Field Measurement of Hybrid Wing Body Model with Blown Flaps," $26^{\text {th }}$ AIAA Applied Aerodynamics Conference, AIAA 2008-6718, August 2008. 
${ }^{18}$ Soderman, P. T., Schmitz, F. H., Allen, C. S., Jaeger, S. M., Sacco, J. N., and Hayes, J. A., "Design of a deep acoustic lining for the 40- by 80-foot Wind Tunnel test section," AIAA-1999-1938, NASA Ames Research Center, Moffett Field, 1999.

${ }^{19}$ Norman, T. R., Shinoda, P. M., Kitaplioglu, C., Jacklin, S. A., and Sheikman, A., "Low-Speed Wind Tunnel Investigation of a Full-Scale UH-60 Rotor System," American Helicopter Society $58^{\text {th }}$ Annual Forum, AD-A480625, June 2002.

${ }^{20}$ Burnside, N. J., Jaeger, S. M., Reinero, B. R., Horne, W. C., and Soderman, P. T., "Array Design and Performance for a Large Scale Airframe Noise Study," $8^{\text {th }}$ AIAA/CEAS Aeroacoustics Conference \& Exhibit, AIAA 2002-2576, June 2002.

${ }^{21}$ Vassberg, J. C., DeHaan, M. A., Rivers, S. M., and Wahls, R. A., "Development of a Common Research Model for Applied CFD Validation Studies," 26th AIAA Applied Aerodynamics Conference, AIAA 2008-6919, August 2008.

${ }^{22}$ Driver, D. M., and Drake, A., "Skin-Friction Measurements Using Oil-Film Interferometry in NASA's 11-Foot Transonic Wind Tunnel," AIAA Journal, Vol. 46, No. 10, October 2008.

${ }^{23}$ Wilcox, D. C., Turbulence Modeling for CFD, Third Edition, DCW Industries, Inc., La Cañada, California, 2006.

${ }^{24}$ Hellsten, A., "New Advanced $k-\omega$ Turbulence Model for High-Lift Aerodynamics," AIAA Journal, vol. 43, no. 9, pp. 1857-1869.

${ }^{25}$ Winter, K. G., "An Outline of the Techniques Available for the Measurement of Skin Friction in Turbulent Boundary Layers," Progress in Aerospace Sciences, Vol. 18, No. 1, 1977, pp. 1-57.

${ }^{26}$ Naughton, J. W., and Sheplak, M., "Modern Developments in Shear-Stress Measurement," Progress in Aerospace Sciences, Vol. 38, No. 1, 2002, pp. 515-570.

${ }^{27}$ Haritonidis J. H., "The measurement of wall shear-stress," Gad-el-Hak M, editor. Advances in fluid mechanics, Berlin: Springer, 1989. pp. 229-61.

${ }^{28}$ Sheplak, M., et al., "MEMS Shear Stress Sensors: Promise and Progress," AIAA Aerodynamic Measurement Technology and Ground Testing Conference, University of Florida, June 2004.

${ }^{29}$ Reda, D. C., and Muratore, J. J., Jr., "Measurement of Surface Shear Stress Vectors Using Liquid Crystal Coatings," AIAA Journal, Vol. 32, No. 8, August 1994.

${ }^{30}$ Carpenter, A. L., Saric, W. S., and Reed, H. L., "In-Flight Receptivity Experiments on a 30-Degree Swept-Wing using Micron-Sized Discrete Roughness Elements," $47^{\text {th }}$ AIAA Aerospace Sciences Meeting, Texas A\&M, College Station Texas, January 2009.

${ }^{31}$ Reda, D. C., Wilder, M. C., Mehta, R. D., and Zilliac, G., "Measurement of Continuous Pressure and Shear Distributions Using Coating and Imaging Techniques," AIAA Journal, Vol. 36, No. 6, June 1998.

${ }^{32}$ Zilliac, G. G., "Further Developments of the Fringe-Imaging Skin Friction Technique," NASA TM110425, Dec. 1996.

${ }^{33}$ Fonov, S., Crafton, J., Forlines, A., and Goss, L., "Demonstration of a Surface Stress Sensitive Films for Skin Friction Measurements in a Variety of Flows," $27^{\text {th }}$ AIAA Aerodynamic Measurement Technology and Ground Testing Conference, AIAA 2010-4800, June 2010.

${ }^{34}$ Wolf, S. W. D., and Laub, J. A., "NASA Ames Laminar Flow Supersonic Wind Tunnel (LFSWT) Tests of a $10^{\circ}$ Cone at Mach 1.6," NASA Technical Memorandum 110438, Ames Research Center, March 1997.

${ }^{35}$ Driver, D. M., "Application of Oil Film Interferometry Skin-Friction to Large Wind Tunnels," NASA Ames Research Center, M/S 229-1, May 1998.

${ }^{36}$ Naughton, J. W., and Brown, J. L., "Uncertainty Analysis for Oil-Film Interferometry Skin-Friction Measurement Techniques," Ames Research Center, 1996.

${ }^{37}$ Monson, D. J., Mateer, G. G., and Menter, F. R., "Boundary-Layer Transition and Global Skin Friction Measurements with an Oil-Fringe Imaging Technique," SAE 932550, Aerotech '93, Costa Mesa, CA, September, 1993.

${ }^{38}$ Zilliac, G. G., "The Fringe-Imaging Skin Friction Technique PC Application User's Manual," NASA/TM-1999-208794, September, 1999.

${ }^{39}$ Driver, D. M., and Zilliac, G. G., "Oil Film Interferometry Shear Stress Measurements in Large Wind Tunnels - Technique and Applications," 24th AIAA Aerodynamic Measurement Technology and Ground Testing Conference, AIAA-2004-2113, June 2004. 
${ }^{40}$ Squire, L. C., "The Motion of a Thin Oil Sheet under the Steady, Boundary Layer on a Body," Journal of Fluid Mechanics, vol. 11, 1961, pp. 161-179.

${ }^{41}$ Tanner, L. H. and Blows, L. G., "A Study of the Motion of Oil Films on Surfaces in Air Flow, with Application to the Measurement of Skin Friction," Journal of Phyiscs E., vol. 9, 1976.

${ }^{42}$ Naughton, J. W., Viken, S., and Greenblatt, D., "Skin-Friction Measurements on the NASA Hump Model," AIAA Journal, vol. 44, no. 6, June 2006, pp. 1255-1265.

${ }^{43}$ Information About Dow Corning Silicone Fluids, Dow Corning Corp., Midland, MI, 1994.

${ }^{44}$ Kline. S. J., and McClintock, F. A., "Describing Uncertainties in Single-Sample Experiments," Mechanical Engineering, January 1953, pp. 3-8.

${ }^{45}$ Decker, R. K. and Naughton, J. W., "Improved Fringe Imaging Skin Friction Analysis Using Automated Fringe Identification," 39th AIAA Aerospace Sciences Meeting and Exhibit, AIAA 2001-0557, Laramie, WY, 82071, January 2001.

${ }^{46}$ Photoshop, Ver. 7.0, Adobe Systems, Inc., San Jose, CA, 2002.

${ }^{47}$ Preston, J. H., "The Determination of Turbulent Skin Friction by Means of Pitot Tubes," Journal of the Royal Aeronautical Society, vol. 58, 1954, pp. 109-121.

${ }^{48}$ Bender, A. M., Drake, A., Westphal, R. V., and Jordan, S. R., "Development and Flight Demonstration of Self-Contained Boundary Layer Measurement Devices," $26^{\text {th }}$ AIAA Applied Aerodynamics Conference, AIAA 2008-7333, August 2008.

${ }^{49}$ Hwang, D., Fralick, G. C., Martin, L. C., Wrbanek, J. D., and Blaha, C. A., "An Innovative Flow Measuring Device: Thermocouple Boundary Layer Rake," NASA/TM—2001-211161, December 2001.

${ }^{50}$ Fralick, G., Wrbanek, J. D., Hwang, D., and Turso, J., "Sensors for Using Times of Flight to Measure Flow Velocities," NASA Technical Briefs, LEW-17944-1, July 2006.

${ }^{51}$ The Mathworks Inc., Natick, MA, MATLAB, 7th ed., 2008.

${ }^{52}$ Poularikas, A. D., and Seely, S., Signals and Systems, PWS Publishers, Boston, 1985.

${ }^{53}$ Thompson, J. F., Soni, B. K., and Weatherill, N. P., Handbook of Grid Generation, CRC Press LLC, Boca Raton, Florida, 1999.

${ }^{54}$ Momentive Performance Materials, "Quartz Thermal Properties," accessed July 24, 2010. $<$ http://www.momentive.com/Internet/Quartz/Properties/ci.Thermal+Properties.props\#>

${ }^{55}$ Miner, D. F., and Seastone, J. B., (ed.), Handbook of Engineering Materials, $1^{\text {st }}$ ed., Wiley Engineering Handbook Series, John Wiley \& Sons, Inc., 1955.

${ }^{56}$ Saric, W. S., "Design of High-Reynolds-Number Flat-Plate Experiements in the NTF," NASA-CR184627, December 1988.

${ }^{57}$ Kendall, J. M., "Boundary Layer Receptivity to Weak Freestream Turbulence," NASA/CP-2007214667, March 2007.

${ }^{58}$ Gand, F., Brunet, V., and Deck, S., "A Combined Experimental, RANS and LES Investigation of a Wing Body Junction Flow," $40^{\text {th }}$ Fluid Dynamics Conference and Exhibit, AIAA 2010-4753, June 2010.

${ }^{59}$ Dorf, R. C., (ed.), The Electrical Engineering Handbook, CRC Press, Inc., Boca Raton, Florida, 1993.

${ }^{60}$ Stanley, W. D., Dougherty, G. R., and Dougherty, R., Digital Signal Processing, $2^{\text {nd }}$ ed., Reston Publishing Company, Inc., Reston, Virginia, 1984. 